\author{
Universidade de São Paulo \\ Instituto de Física
}

\title{
Natureza da transição de fase quark-hádron e consequências para a estrutura estelar
}

\author{
Milena Bastos Albino
}

Orientador: Prof. Dr. Fernando Silveira Navarra

Dissertação de mestrado apresentada ao Instituto de Física da Universidade de São Paulo, como requisito parcial para a obtenção do título de Mestra em Ciências.

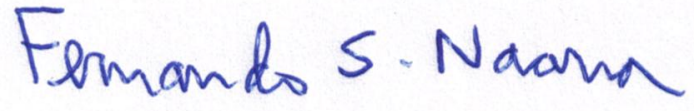

Banca Examinadora:

Prof. Dr. Fernando Silveira Navarra - Orientador (IFUSP)

Profa. Dra. Débora Peres Menezes (UFSC)

Profa. Dra. Maria Constança Mendes Pinheiro da Providência Santarém e Costa (Univ. de Coimbra) 
FICHA CATALOGRÁFICA

Preparada pelo Serviço de Biblioteca e Informação do Instituto de Física da Universidade de São Paulo

Albino, Milena Bastos

Natureza da transição de fase quark-hádron e consequências para a estrutura estelar. São Paulo, 2021.

Dissertação (Mestrado) - Universidade de São Paulo. Instituto de Física. Depto. de Física Nuclear

Orientador(a): Prof. Dr. Fernando Silveira Navarra

Área de Concentração: Física de Partículas

Unitermos: 1. Estrelas de Neutrons; 2. Física de Partículas; 3. Cromodinâmica Quântica.

USP/IF/SBI-084/2021 
University of São Paulo

Physics Institute

\title{
Nature of the quark-hadron phase transition and consequences for the star structure
}

\author{
Milena Bastos Albino
}

Orientador: Prof. Dr. Fernando Silveira Navarra

Dissertation submitted to the Physics Institute of the University of São Paulo in partial fulfillment of the requirements for the degree of Master of Science.

Examining Committee:

Prof. Dr. Fernando Silveira Navarra - Supervisor (IFUSP)

Prof. Dr. Débora Peres Menezes (UFSC)

Prof. Dr. Maria Constança Mendes Pinheiro da Providência Santarém e Costa (Univ. de Coimbra) 



\section{Agradecimentos}

Este trabalho jamais teria sido realizado sem todo o apoio, ajuda e motivação que recebi de tantas pessoas.

Por isso, agradeço aos meus pais, por todo o amor que me deram e que me impulsionou ao longo de toda a minha a vida. À toda minha família, por todo o carinho e pelos ótimos momentos juntos. Por sempre estarem por perto e desejarem o melhor para mim.

Agradeço ao Renato, que mesmo à distância, está sempre comigo, me dando apoio e alegrando os meus dias. Por sempre arranjar um tempo para conversar e me dar forças para continuar.

Um grande agradecimento ao Fernando Navarra, meu orientador, pela dedicação, motivação e seu ótimo humor. Por ter acreditado em mim e me dado a possibilidade de estudar uma área tão bonita e tão promissora.

Ao Ricardo Fariello, pela ajuda e disposição que sempre demonstrou, e ao Germán Lugones, pelas conversas proveitosas.

Aos meus amigos, Fernanda, Chiara, Teresa, Fernando, Carol, Cauê, Gustavo, e outros tantos que sempre me incentivam e deixam meus dias mais leves.

Por fim, à CNPq, pelo apoio financeiro.

O presente trabalho foi realizado com apoio do CNPq, Conselho Nacional de Desenvolvimento Científico e Tecnológico - Brasil. 



\section{Resumo}

As estrelas de nêutrons são o segundo objeto mais denso do universo (apenas menos densos do que os buracos negros) e possuem uma temperatura relativamente baixa. Por causa destas características, a matéria de que são compostas estas estrelas compactas está numa região do diagrama de fases da QCD que permanece pouco compreendida. Assim, as estrelas de nêutrons funcionam como laboratórios para o estudo da matéria de alta densidade e baixa temperatura.

Neste trabalho, partindo das equações da MFTQCD (em inglês, Mean-Field Theory of Quantum ChromoDynamics) obtemos o diagrama massa-raio e a deformabilidade de maré. Comparando esses resultados com os dados experimentais, concluímos que a MFTQCD é uma boa alternativa para descrever as propriedades conhecidas das estrelas de nêutrons. Porém, os novos dados experimentais impõem maior restrição aos valores dos parâmetros livres. Em seguida, estudamos quais as consequências que a natureza da transição de fase implica nas características das estrelas de nêutrons. Usando uma equação de estado que combina a fase hadrônica e de quarks, controlamos a suavidade desta transição variando o valor de um parâmetro livre $\rho_{c}$. Os nossos resultados indicam que transições de fase mais abruptas apresentam maior compatibilidade com os dados experimentais.

Palavras-chaves: estrelas de nêutrons; estrelas híbridas; diagrama de fases da QCD; matéria de quarks. 



\section{Abstract}

Neutron stars are the second most dense objects in the universe (only less dense than black holes) and have a relatively low temperature. Because of these features, neutron star matter lies in a poorly understood region of the QCD phase diagram. Neutron stars can work as laboratories for the study of cold and dense matter.

In this work, we used the MFTQCD (Mean-Field Theory of Quantum Chromodynamics) and we obtained the mass-radius diagram and the tidal deformability. Comparing these results with the experimental data, we conclude that the MFTQCD remains a good model to describe the properties of neutron stars; however, the parameter window is now narrower. Next, we studied the impact of the nature of the phase transition on neutron star properties. We used an equation of state which combines hadronic matter and quark matter. We control the smoothness of the quark-hadron transition by varying the free parameter $\rho_{c}$. Our results indicate that a sharp phase transition is more compatible with experimental data.

Keywords: neutron stars; hybrid stars; QCD phase diagram; quark matter. 



\section{Lista de Figuras}

1.1 Diagrama de fase da QCD em função da temperatura e do potencial químico. Imagem retirada de $[1] \ldots \ldots \ldots \ldots 2$

1.2 Equação de estado da QCD em temperatura nula. À esquerda, temos os resultados obtidos através da teoria efetiva quiral (cEFT) [3], e à direita, os resultados obtidos pela teoria de perturbação (pQCD) [7]. . . . . . . . . . . 3

1.3 Representação da evolução estelar. Imagem retirada de [1]. . . . . . . . . . . 3

1.4 Representação de possíveis modelos para o interior de uma estrela de nêutrons. Imagem retirada de $[22] \ldots \ldots$. . . . . . . . . . . . 5

1.5 Dados experimentais do diagrama massa-raio. . . . . . . . . . . . . 6

2.1 Pressão central utilizada em quatro pontos do diagrama de massa-raio. . . . . . 10

3.1 Pressão (à esquerda) e densidade de energia (à direita) em função da densidade bariônica do modelo de sacola do MIT. . . . . . . . . . . . . . . . . . . 19

3.2 Pressão em função da densidade de energia do modelo de sacola do MIT. . 19

3.3 Valores de $\epsilon / \rho_{B}$ em função de $B$ para a matéria de quark (em vermelho) e para a matéria estranha (em azul). Em cinza, temos $\epsilon /\left.\rho_{B}\right|^{56} \mathrm{Fe}=934 \mathrm{MeV}$. . 20

3.4 Relação massa-raio à esquerda e deformabilidade de maré à direita para uma estrela de quarks cujos constituintes são descritos pelo modelo de sacola do MIT . . . . . . . . . . . . . . . . . . . . .

3.5 Diagramas de Feynamn da equação (3.2.20). Linhas contínuas representam os quarks; linhas tracejadas, os hard glúons; e linhas helicoidais, os soft glúons [52]. . . . . . . . . . . . . . . . . . . . 26

3.6 Gráfico da estabilidade da MFTQCD. . . . . . . . . . . . . . . . . . . 32

3.7 Pressão em função da densidade de energia. As linhas coloridas são os gráficos das equações de MFTQCD e a linha traço-ponto em preto, é o modelo de comparação apresentado em [20]. . . . . . . . . . . . . . . . . . . . . 33 
3.8 Relação massa-raio à esquerda e deformabilidade de maré à direita para uma estrela de quarks cujos constituintes são descritos pela MFTQCD. . . . . . . 33

4.1 Pressão (à esquerda) e densidade de energia (à direita) em função da densidade bariônica do modelo do gás de Fermi.

4.2 Pressão em função da densidade de energia do modelo do gás de Fermi. Em preto, temos o resultado obtido pela teoria efetiva quiral [3].

4.3 Relação massa-raio à esquerda e deformabilidade de maré à direita para uma estrela de nêutrons cujos constituintes são descritos pelo modelo de gás de Fermi. O diagrama massa-raio obtido está compatível com o resultado de Oppenheimer e Volkoff em 1939 [9]. . . . . . . . . . . . . . . . . . . . . . . 38

4.4 Pressão em função da densidade de massa do modelo HLPS. Para valores menores de $\rho_{1}$, temos uma comparação da equação de estado retirada de [60] (em vermelho) com a banda obtida pela cEFT (em azul). Para valores maiores que $\rho_{1}$, temos as três equações politrópicas. Imagem retirada de [3] . . . . . . 38

4.5 Pressão (à esquerda) e densidade de energia (à direita) em função da densidade bariônica do modelo de HLPS. . . . . . . . . . . . . . . . . . . . . 40

4.6 Pressão em função da densidade de energia do modelo de HLPS. Em preto, temos o limite de baixa densidade obtido através da teoria efetiva quiral (cEFT)

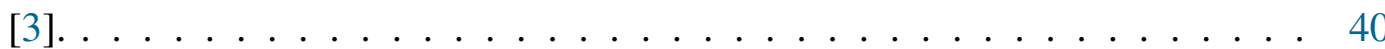

4.7 Relação massa-raio à esquerda e deformabilidade de maré à direita para uma estrela de nêutrons cujos constituintes são descritos pelo modelo do HLPS. . . 40

5.1 Pressão em função da densidade bariônica com o valor de $\delta_{0}=100\left(\mathrm{MeV} / \mathrm{fm}^{3}\right)^{2}$ para diferentes valores de $\rho_{c} \ldots \ldots \ldots \ldots \ldots$

5.2 Pressão em função da densidade bariônica com o valor de $\rho_{c}=0.5 \mathrm{fm}^{-3}$ para diferentes valores de $\delta_{0} \ldots \ldots \ldots \ldots \ldots$. . . . . . . . . . . . 44

5.3 Pressão (à esquerda) e densidade de energia (à direita) em função da densidade bariônica. As linhas contínuas representam as equações híbridas MIT + Fermi com os valores de $\rho_{c}=0.2$, em vermelho; $\rho_{c}=0.4$, em verde; $\rho_{c}=1.0$, em azul. A linha tracejada representa a equação de estado do modelo de sacola do MIT, enquanto que a linha pontilhada, o modelo de gás de

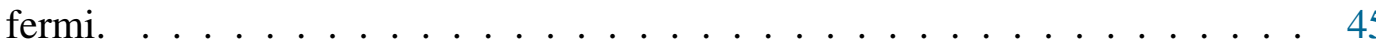

5.4 Pressão em função da densidade de energia. As linhas contínuas representam as equações híbridas MIT + Fermi, com os valores de $\rho_{c}=0.2$, em vermelho; $\rho_{c}=0.4$, em verde; $\rho_{c}=1.0$, em azul. A linha tracejada representa a equação de estado do modelo de sacola do MIT, enquanto que a linha pontilhada, o modelo de gás de Fermi. . . . . . . . . . . . . . . . . . . . . . . . . 4 
5.5 Diagrama massa-raio (à esquerda) e deformabilidade de maré em função da massa da estrela (à direita) para uma estrela de híbrida cujos constituintes são descritos pela equação de estado MIT + Fermi. . . . . . . . . . . . . . . 46

5.6 Pressão em função da densidade bariônica. Na primeira linha, temos os gráficos com o HLPS Soft; na segunda, com o HLPS Inter; na terceita, com o HLPS Stiff. Na primeira coluna, temos os gráficos com a MFTQCD set III; na segunda, com o set II; na terceira, com o set I. . . . . . . . . . . . . . 47

5.7 Pressão em função da densidade bariônica do modelo MFTQCD + HLPS. . . 49

5.8 Pressão em função da densidade de energia do modelo MFTQCD + HLPS. . . 49

5.9 Diagrama massa-raio (à esquerda) e deformabilidade de maré em função da massa da estrela (à direita) para uma estrela de híbrida cujos constituintes são descritos pela equação de estado MFTQCD + HLPS, com o conjunto III da equação da MFTQCD e a equação soft de HLPS. . . . . . . . . . . . . . . . 50

5.10 Diagrama massa-raio (à esquerda) e deformabilidade de maré em função da massa da estrela (à direita) para uma estrela de híbrida cujos constituintes são descritos pela equação de estado MFTQCD + HLPS, com o conjunto III da equação da MFTQCD e a equação intermediate de HLPS. . . . . . . . . . . . 50

5.11 Diagrama massa-raio do artigo [17]. A linha preta contínua representa a estrela de nêutrons constituída inteiramente por hádrons e as linhas coloridas, são as estrelas híbridas com diferentes escolhas de parâmetros. . . . . . . . . 52 



\section{Lista de Tabelas}

3.1 Massa e carga elétrica das partículas presentes na fase de quarks. . . . . . . . 18

3.2 Janela de estabilidade dos parâmetros livres da MFTQCD. . . . . . . . . . . 32

4.1 Valores dos parâmetros das equações de estado do modelo HLPS modificado. 39

5.1 Densidade bariônica em que ocorre a transição de fase para cada combinação

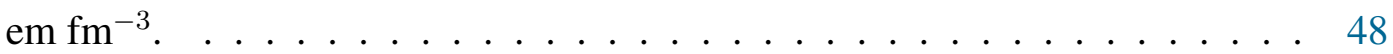

B.1 Exemplo de resultado obtido ao final de todo o processo de resolução numérica da TOV. . . . . . . . . . . . . . . . . . . . 63 



\section{Sumário}

Lista de Figuras vii

Lista de Tabelas

Sumário $\quad$ xiii

1 Introdução 1

1.1 Diagrama de Fases da QCD . . . . . . . . . . . . . . . . . . . . 1

1.2 Estrelas de Nêutrons . . . . . . . . . . . . . . . . . . . . . 2

1.3 Objetivos deste Trabalho . . . . . . . . . . . . . . 6

2 Propriedade das Estrelas de Nêutrons 9

2.1 Equações de Tolman-Oppenheimer-Volkoff (TOV) . . . . . . . . . . 9

2.2 Deformabilidade de Maré . . . . . . . . . . . . . . . . . . . . . 11

3 Estrelas de Quarks 13

3.1 Modelo de Sacola do MIT . . . . . . . . . . . . . . . . . . . . . . . . . 14

3.2 QCD em Campo Médio (MFTQCD) . . . . . . . . . . . . . . . 21

4 Estrelas de Hádrons $\quad 35$

4.1 Modelo de Gás de Fermi . . . . . . . . . . . . . . . . . . . . . . 35

4.2 Modelo de HLPS . . . . . . . . . . . . . . . . . . . . . 38

5 Estrelas Híbridas $\quad 41$

5.1 Intensidade da Transição de Fase . . . . . . . . . . . . . . . . . . 41

6 Conclusão 
A Dedução da TOV

A.1 Equação da Massa . . . . . . . . . . . . . . . . . . . . . . . . 55

A.2 Equação da Pressão . . . . . . . . . . . . . . . . . . . . 56

B Resolução Numérica da TOV

C Deformabilidade de Maré $\quad 65$

C.1 Potencial Gravitacional Newtoniano . . . . . . . . . . . . . . 65

C.2 Tidal Love Number $k_{2} \ldots \ldots \ldots$. . . . . . . . . . . . . . . . 67

D Revisão da QCD $\quad 75$

D.1 Equação $(3.2 .39) \quad \ldots \ldots \ldots$. . . . . . . . . . . . . . . 76

D.2 Equação $(3.2 .37) \ldots \ldots \ldots$. . . . . . . . . . . . . . 77

D.3 Equação $(3.2 .45) \quad \ldots \ldots \ldots$. . . . . . . . . . . . . . . 79

$\begin{array}{lr}\text { Bibliografia } & 83\end{array}$ 


\section{Introdução}

\subsection{Diagrama de Fases da QCD}

Atualmente, acreditamos que os fenômenos da Natureza podem ser descritos através de quatros forças fundamentais: a força gravitacional, a eletromagnética, a força forte e a força fraca. Neste trabalho, iremos buscar uma maior compreensão da força forte. No nível mais fundamental, esta força é sentida pelos quarks e glúons, que são as únicas partículas a possuírem a carga da força forte, denominada por carga de cor. A Cromodinâmica Quântica (QCD - em inglês, Quantum Chromodynamics) é a teoria que descreve a interação forte no nível mais fundamental. Apesar da lagrangiana da QCD ser conhecida e ser simples, ainda não é possível obter uma descrição completa da matéria em todo o diagram de fase. A compreensão deste diagrama é hoje um dos grandes desafios para a QCD (ver figura 1.1).

Uma região muito estudada do diagrama de fases da QCD é a região de alta temperatura e baixo potencial químico. Estas condições são reproduzidas experimentalmente através dos aceleradores de partículas, onde já observamos [2] que a matéria hadrônica se transforma no plasma de quarks e glúons (QGP - em inglês, Quark Gluon Plasma). Este processo de desconfinamento dos quarks pode ocorrer também na região de baixa temperatura e alto potencial químico. Neste caso, dizemos que a matéria hadrônica se converte no que chamamos de QGP frio ou matéria de quarks. Neste trabalho, vamos estudar esta região do diagrama de fases da QCD.

Quando a temperatura é nula, a região de baixa densidade bariônica é bem conhecida. Nessas condições, podemos descrever o comportamento da matéria através da teoria de campo efetiva quiral [3] (cEFT - em inglês, chiral effective field theory). Já na região de alta densidade ( $\rho_{B} \gtrsim 40 \rho_{0}$ [4], onde $\rho_{0}=0.16 \mathrm{fm}^{-3}$ é a densidade de saturação nuclear), é possível obter o comportamento da matéria através dos cálculos da teoria da QCD per- 


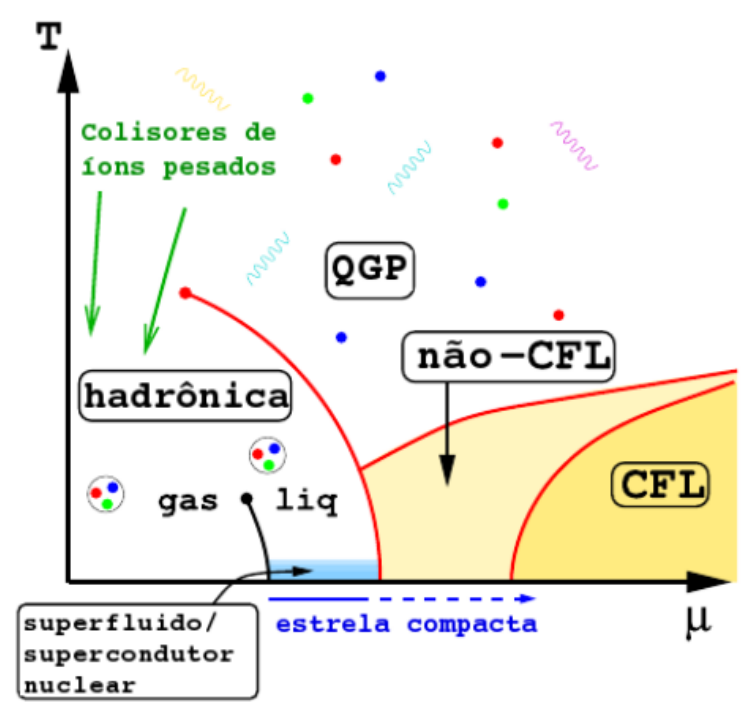

Figura 1.1: Diagrama de fase da QCD em função da temperatura e do potencial químico. Imagem retirada de [1].

turbativa [5, 6] (pQCD - em inglês, perturbative QCD). Entretanto, entre as regiões de alta e baixa densidade, não há, atualmente, aproximações possíveis que nos permitam obter resultados a partir da QCD. Desta forma, temos uma região desconhecida, como mostrado na figura 1.2. Para obter uma boa compreensão desta região, usamos modelos que reproduzem as características esperadas da matéria. Com estes modelos, podemos obter as equações de estado (EoS - em inglês, equation of state) e, comparando com dados experimentais, podemos concluir se eles fornecem uma boa descrição do QGP frio. As condições de baixa temperatura e alto potencial químico são de difícil reprodução em laboratórios. Por outro lado, existe um corpo celeste que naturalmente reproduz estas condições desejadas. Este corpo é o que chamamos de estrela de nêutrons. Na última década, ocorreu um grande avanço na determinação das propriedades das estrelas de nêutrons, principalmente após ser possível a detecção de ondas gravitacionais. Desta forma, o rápido desenvolvimento experimental nos dá uma ótima motivação para o estudo das estrelas de nêutrons e do QGP frio.

\subsection{Estrelas de Nêutrons}

As estrelas se mantém estáveis através do equilíbrio entre a força gravitacional e a pressão gerada pela fusão de átomos [8]. Entretanto, em um dado momento, esse combustível irá acabar, de forma que a estrela se colapsará [1]. Ao final desse processo, essa estrela se transformará em uma anã branca, caso $M_{\text {inicial }} \lesssim 8 M_{\odot}$, em uma estrela de nêutrons, caso $M_{\text {inicial }} \lesssim 25 M_{\odot}$, ou em um buraco negro, caso $M_{\text {inicial }} \gtrsim 25 M_{\odot}$ [7] (ver figura 1.3). Neste 


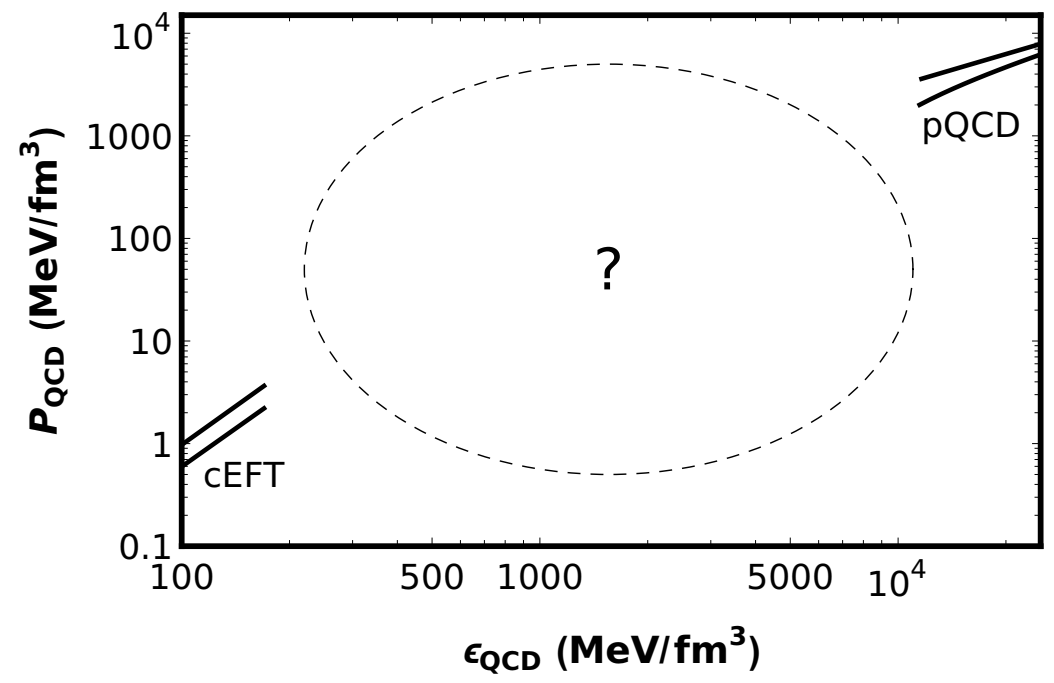

Figura 1.2: Equação de estado da QCD em temperatura nula. À esquerda, temos os resultados obtidos através da teoria efetiva quiral (cEFT) [3], e à direita, os resultados obtidos pela teoria de perturbação (pQCD) [7].

trabalho, vamos estudar estrelas de nêutrons. Neste caso, sem a pressão gerada pela fusão do hidrogênio, a quantidade que equilibra a força gravitacional é a pressão gerada pelo princípio de Pauli. Como consequência, estrelas de nêutrons se tornam objetos relativamente frios e que possuem uma densidade muito alta, reproduzindo as características da região desconhecida do diagrama de fases de QCD para alta densidade bariônica e baixa temperatura.

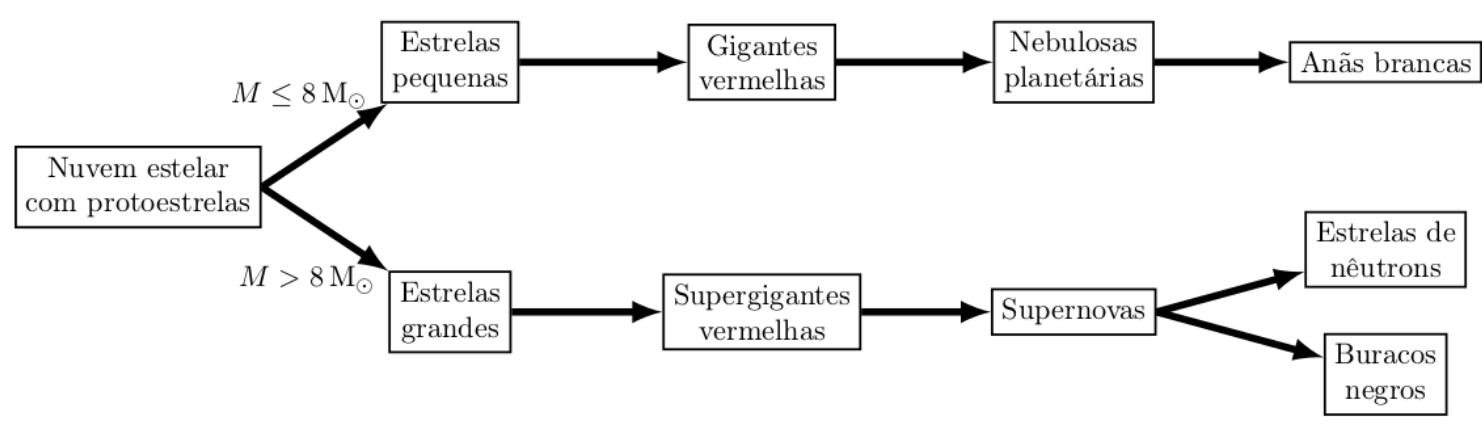

Figura 1.3: Representação da evolução estelar. Imagem retirada de [1].

Tendo agora esse conhecimento, podemos nos perguntar como efetuamos, na prática, a conexão entre um modelo do QGP frio e as estrelas de nêutrons. A ideia geral é que, primeiramente, supomos que as estrelas de nêutrons são esféricas, estáticas e constituídas de um fluido ideal e isotrópico. A partir destas hipóteses, podemos resolver as equações de Einstein e obter o que chamamos de equações de Tolman-Oppenheimer-Volkoff (TOV) 
$[9,10]$, dadas por

$$
\begin{aligned}
\frac{d P}{d r} & =-\frac{G \epsilon(r) M(r)}{r^{2}}\left[1+\frac{P(r)}{\epsilon(r)}\right]\left[1+\frac{4 \pi r^{3} P(r)}{M(r)}\right]\left[1-\frac{2 G M(r)}{r}\right]^{-1}, \\
\frac{d M}{d r} & =4 \pi r^{2} \epsilon(r) .
\end{aligned}
$$

onde $P(r), \epsilon(r), M(r)$ e $r$ são a pressão, a densidade de energia, a massa e o raio, respectivamente. Desta forma, a estrutura macroscópica das estrelas de nêutrons é obtida por este sistema de equações diferenciais. Podemos ver que este sistema depende de três funções, porém, ele nos fornece apenas duas equações diferenciais. Com isso, para resolver estas equações, é necessário fornecer mais informação, como por exemplo, uma equação que relacione a pressão com a densidade de energia. Essa relação pode ser obtida através de um modelo que descreve o QGP frio. Assim, a conexão entre a estrutura microscópica com a estrutura macroscópica é feita. Em resumo: partindo de um modelo, obtermos as equações de estado da matéria, nos permitindo resolver as equações de TOV. A resolução destas equações nos fornece características das estrelas de nêutrons, que podemos comparar com os dados experimentais. Desta forma, por eliminação, conseguimos selecionar os modelos que produzem uma boa descrição da matéria nas condições de baixa temperatura e alta densidade e nos aproximar de um melhor entendimento tanto sobre a força forte, quanto sobre as estrelas de nêutrons.

Até aqui, consideramos que as estrelas de nêutrons são constituídas inteiramente por matéria de quarks. Neste caso, elas são chamadas de estrelas de quarks [11, 12, 13, 14, 15]. Entretanto, não há um consenso em relação a fase em que a matéria se apresenta no interior das estrelas de nêutrons. Poderia ocorrer que as estrelas de nêutrons não apresentassem densidade suficiente para desconfinar a matéria. Nesta situação, elas seriam formadas inteiramente por matéria na fase hadrônica e seriam chamadas de estrelas de hádrons $[3,16]$. Uma outra possibilidade é o que chamamos de estrelas híbridas $[17,18$, 19, 20,21]. As estrelas híbridas seriam constituídas de matéria de quarks em seu interior (onde há uma densidade maior da matéria) e estariam envolvidas por uma camada de matéria hadrônica (densidade menor). Podemos ver diferentes formas de modelar estrelas de nêutrons na figura 1.4.

Conforme comentamos anteriormente, é através de dados observacionais que podemos verificar se o modelo utilizado faz uma boa descrição ou não da matéria fria e densa. Na última década, houve grandes avanços experimentais em relação à detecção das estrelas de nêutrons. Quanto maior a quantidade de dados observacionais que tivermos, maior será a quantidade de condições que os modelos utilizados devem satisfazer. Com isso, os modelos irão sendo selecionados, e a precisão da descrição da matéria aumentando. Atualmente, as condições que os modelos das estrelas de nêutrons devem satisfazer são 


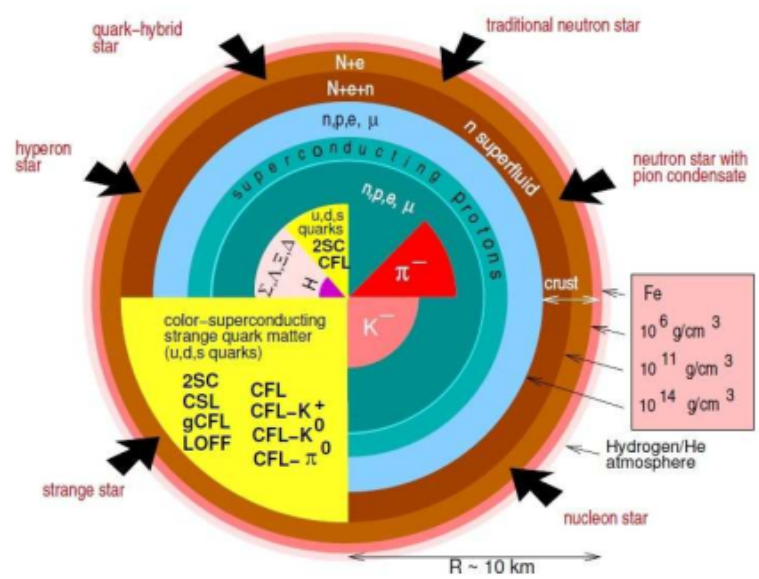

Figura 1.4: Representação de possíveis modelos para o interior de uma estrela de nêutrons. Imagem retirada de [22].

[4]:

- Massa máxima deve ser maior do que $2 M_{\odot}$.

Na última década, as medidas feitas com a técnica conhecida como Shapiro delay revelaram vários pulsares com massa próxima ou superior a $2 M_{\odot}$. Em 2010, foi constatado que a massa do pulsar J1614-2230 era de $M=1.97_{-0.04}^{+0.04} M_{\odot}$ [23]; porém, em 2018, uma atualização destes dados resultou em uma massa de $M=1.908_{-0.016}^{+0.016} M_{\odot}$ [24]. Em 2013, foi obtida a massa do pulsar J0348+0432, sendo ela de $M=2.01_{-0.04}^{+0.04} M_{\odot}$ [25]. Em 2019, a medida do pulsar J0740+6620 resultou em $M=2.14_{-0.09}^{+0.10} M_{\odot}(68.3 \%$ de nível de credibilidade) [26]. A mais recente medida de um pulsar com massa próxima de $2 M_{\odot}$, realizada pela colaboração NICER em 2021, levou à conclusão de que o pulsar J0740+6620 tem massa $M=2.072_{-0.066}^{+0.067} \mathrm{M}_{\odot}$ e raio $R=12.39_{-0.98}^{+1.30} \mathrm{~km} \mathrm{[27].} \mathrm{Esta} \mathrm{medida} \mathrm{simultânea} \mathrm{da}$ massa e do raio, define um ponto de referência no diagrama massa-raio (ponto 4 da figura $1.5)$.

Por outro lado, existe a possibilidade deste valor de massa máxima aumentar. LIGO e Virgo, em 2020, detectaram ondas gravitacionais GW190814 geradas pelo sistema binário formado por um buraco negro e um objeto compacto. A medida indica que a massa deste último é de $2.5-2.67 M_{\odot}$ [28]. Entretanto, não é consenso se o objeto mais leve é uma estrela de nêutrons ou um buraco negro muito leve [29, 30]. O valor mínimo da massa máxima da possível estrela de nêutrons é destacado nos diagramas de massa-raio deste trabalho, como indicado pela figura 1.5.

- Raio de uma estrela com $M=1.4 M_{\odot}$ deve ser de aproximadamente $13 \mathrm{~km}$.

Esta condição vem das medidas obtidas do pulsar J0030+0451. Os valores obtidos pelo 
NICER em 2019 foram de $M=1.44_{-0.14}^{+0.15} M_{\odot} \operatorname{com} R=13.02_{-1.06}^{+1.24} \mathrm{~km} \mathrm{[31],} M=$ $1.34_{-0.16}^{+0.15} M_{\odot} \operatorname{com} R=12.71_{-1.19}^{+1.14} \mathrm{~km}$ [32] e $R_{1.4 M_{\odot}}>10.4 \mathrm{~km}$ [33]. Estes dados são destacados nos gráficos deste trabalho, sendo eles os pontos 3,2 e 1 da figura 1.5 , respectivamente.

- Deformabilidade de maré de uma estrela com $M=1.4 M_{\odot}$ deve ser $<580$.

Em 2017, o experimento LIGO e Virgo detectou as ondas gravitacionais GW170817 do sistema binário de duas estrelas de nêutrons. $O$ valor obtido da detecção foi de massas de $\approx 1.4 M_{\odot}$ com um valor de deformabilidade de maré de $70 \leq \Lambda_{1.4 M_{\odot}} \leq 580$, em um nível de credibilidade de $90 \%$ [34, 35]. Desta forma, esse dado experimental restrige os modelos teóricos a partir da deformabilidade de maré. Com isso, em todos os modelos testados neste trabalho, iremos calcular o valor de $\Lambda_{1.4 M_{\odot}}$ e compará-lo com o valor experimental obtido pelo LIGO e Virgo.

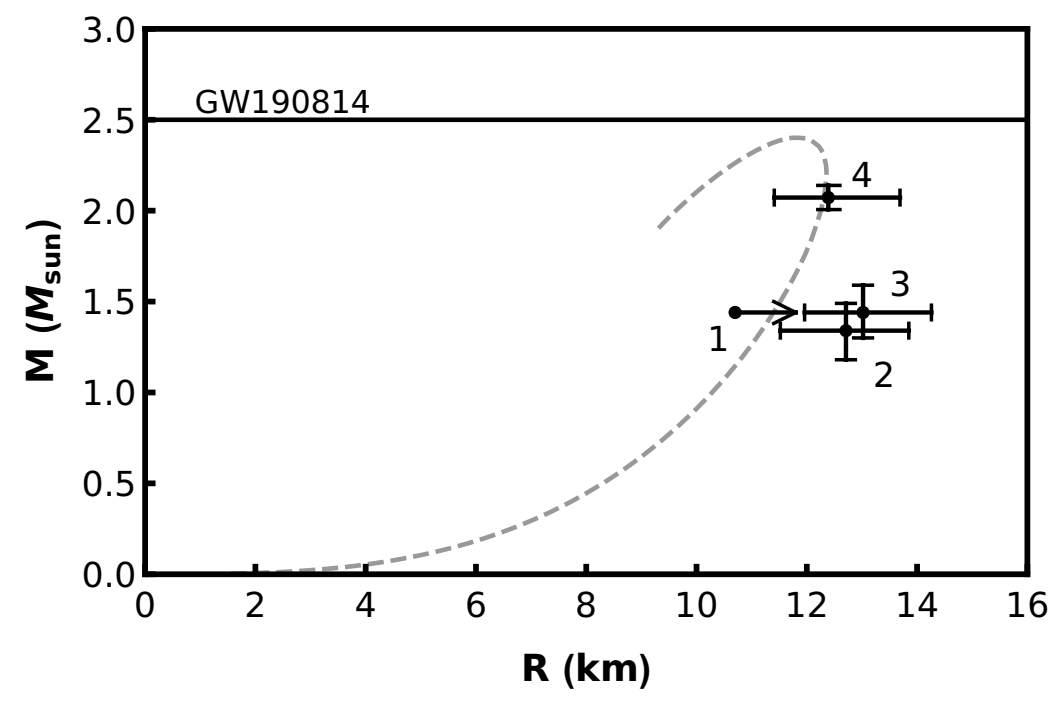

Figura 1.5: Dados experimentais do diagrama massa-raio.

ponto 1: PSR J0030+0451 $\rightarrow R_{1.4 M_{\odot}}>10.4 \mathrm{~km} \mathrm{[33]}$

ponto 2: PSR J0030+0451 $\rightarrow M=1.34_{-0.16}^{+0.15} \mathrm{M}_{\odot}, R=12.71_{-1.19}^{+1.14} \mathrm{~km} \mathrm{[32]}$

ponto 3: PSR J0030+0451 $\rightarrow M=1.44_{-0.14}^{+0.15} \mathrm{M}_{\odot}, R=13.02_{-1.06}^{+1.24} \mathrm{~km} \mathrm{[31]}$

ponto 4: PSR J0740+6620 $\rightarrow M=2.072_{-0.066}^{+0.067} \mathrm{M}_{\odot}, R=12.39_{-0.98}^{+1.30} \mathrm{~km}$ [27]

\subsection{Objetivos deste Trabalho}

Em vista dos rápidos avanços observacionais, vamos inicialmente verificar se a MFTQCD (em inglês, Mean-Field Theory Quantum ChromoDynamics) [36] é capaz de descrever os novos dados experimentais. Para isso, vamos considerar uma estrela de quarks consti- 
Natureza da transição de fase quark-hádron e consequências para a estrutura estelar

tuída de matéria descrita por esse modelo e obter os gráficos da relação massa-raio e da deformabilidade de maré em função da massa. Em seguida vamos compará-los com os dados observacionais e verificar se a MFTQCD satisfaz as novas restrições impostas pelas observações. Esta primeira parte do trabalho resultou no artigo publicado em [11]. Em seguida, nos dedicamos ao estudo de estrelas híbridas.

Quando estudamos a termodinâmica da QCD a temperatura finita e potencial químico zero (densidade bariônica nula), podemos obter resultados rigorosos usando o formalismo e a técnica da QCD na rede (“Lattice QCD” ou LQCD). Com LQCD, é possível fazer uma descrição unificada das duas fases da teoria, desconfinada (QGP) e confinada (gás de hadrons), e da transição entre elas. Após décadas de estudos e refinamentos da LQCD, foi possível determinar com boa precisão a temperatura de transição de fase e sua natureza de "cross-over", ou seja, uma mudança de fase bastante suave. É importante ressaltar que este mesmo tipo de cálculo com QCD na rede não pode ser feito no caso de temperatura zero e potencial químico grande, que são as características das estrelas de nêutrons. Dessa forma, se existirem estrelas híbridas, as duas fases em seu interior (e a mudança de fase) não podem ser descritas pela mesma teoria. Assim, temos que recorrer a modelos. Em geral, o que é feito é juntar um modelo hadrônico com interações realistas (e que descrevem bem a matéria hadrônica a baixas densidades) com um modelo de matéria de quarks com o limite assintótico correto em altas densidades.

Na segunda parte deste trabalho, vamos verificar se é possível extrair dos dados experimentais mais recentes alguma informação sobre a natureza da transição de fase quarkhadron, supondo que as estrelas de nêutrons sejam estrelas híbridas. Para isto, vamos usar um conjunto de equações de estado realistas para cada uma das fases, conectando as equações de estado de cada fase com um parâmetro livre que determina a intensidade da transição e que pode ser determinado através da análise dos dados.

Como ponto de partida para guiar a intuição, escolhemos o modelo de sacola do MIT para a fase de quarks e o modelo de gás de Fermi para a fase de hádrons. O próximo passo será usar modelos mais realistas. Usamos a MFTQCD para a fase de quarks e o modelo que denominamos por HLPS [3] para a fase de hádrons. Neste trabalho, vamos dar uma atenção especial à forma como ocorre essa transição (se ela é uma transição mais abrupta, ou mais suave). Para isso, nos inspiramos nas equações de estado propostas em [37, 38], que nos permitem controlar a natureza da transição a partir da escolha de um parâmetro $\left(\rho_{c}\right)$. Analisando os gráficos de massa-raio e da deformabilidade de maré, identificamos os efeitos que a natureza desta transição de fase exerce sobre a estrela de nêutrons. Por fim, colocamos à prova o modelo de estrela híbrida verificando se ele reproduz as informações observacionais conhecidas até agora. 


\section{Organização da Dissertação}

Essa dissertação está organizada da seguinte forma: no capítulo 2, fazemos uma revisão sobre a estrutura macroscópica das estrelas de nêutrons. Discutimos as equações de Tolman-Oppenheimer-Volkoff (TOV) e a deformabilidade de maré. No capítulo 3, estudamos modelos que descrevem a matéria na fase de quarks, sendo eles o modelo de sacola do MIT e a MFTQCD. Para os dois modelos, resolvemos as equações de TOV e, obtendo o diagrama massa-raio e a deformabilidade de maré, comparamos os resultados com os dados experimentais. No capítulo 4, repetimos o mesmo procedimento do capítulo anterior para modelos que descrevem a matéria na fase de hádrons. Os modelos discutidos foram o modelo de gás de Fermi e o modelo que chamamos de HLPS, retirado de [3]. No capítulo 5, estudamos a equação de estado composta [37, 38] que nos permite determinar a intensidade da transição de fase a partir do valor de um parâmetro livre. Em seguida, aplicamos essa equação de estado composta usando a EoS da fase de quarks do modelo de sacola do MIT e a EoS da fase de hádrons dada pelo modelo de gás de Fermi. Por fim, repetimos o mesmo processo substituindo a EoS do MIT pela da MFTQCD e a EoS do gás de Fermi pela do modelo de HLPS. No capítulo 6, se encontram as conclusões deste trabalho.

\section{Notação}

Neste trabalho, usamos as unidades naturais definidas como $c=\hbar=k_{B}=1$, e a métrica do espaço-tempo plano sendo $g_{\mu \nu}=\operatorname{diag}(+1,-1,-1,-1)$. 


\section{Propriedade das Estrelas de Nêutrons}

\subsection{Equações de Tolman-Oppenheimer-Volkoff (TOV)}

As equações de Tolman-Oppenheimer-Volkoff (TOV) são equações que descrevem a variação da pressão e da massa em função do raio de uma estrela. Sob a hipótese dessa estrela ser esférica, estática, e constituída de um fluido ideal e isotrópico, é possível obter as equações de TOV a partir da equação de Einstein ${ }^{1}$. Elas são:

$$
\frac{d P}{d r}=-\frac{G \epsilon(r) M(r)}{r^{2}}\left[1+\frac{P(r)}{\epsilon(r)}\right]\left[1+\frac{4 \pi r^{3} P(r)}{M(r)}\right]\left[1-\frac{2 G M(r)}{r}\right]^{-1},
$$

onde

$$
\frac{d M}{d r}=4 \pi r^{2} \epsilon(r)
$$

As equações de TOV são deduzidas usando apenas argumentos relacionados à estrutura macroscópica da matéria e são a base para o cálculo das propriedades das estrelas de nêutrons, como a relação massa-raio e a sua deformabilidade de maré. Os resultados dos cálculos podem ser comparados com valores observacionais. Notemos que as equações de TOV são duas equações diferenciais que dependem de três funções $(P(r), \epsilon(r), M(r))$; ou seja, para resolver esse sistema, precisamos de mais uma equação que relacione essas funções. Para isso, é necessário ter um conhecimento externo à estrutura macroscópica da estrela. A partir de modelos que descrevem a composição microscópica, podemos obter uma equação de estado $(\epsilon=\epsilon(P))$. Substituindo nas equações de TOV, passamos a ter duas equações diferenciais com duas funções $(P(r), M(r))$. Assim é possível resolver este sistema e obter as características de uma estrela formada por um fluido descrito a partir da equação de estado utilizada.

\footnotetext{
${ }^{1}$ Uma dedução destas equações é apresentada no apêndice A.
} 
Com as equações de TOV e a equação de estado, a solução deste sistema de equações pode ser obtida numericamente. Para encontrar esta solução numérica, é necessário também saber as condições de contorno. Assim, escolhemos os valores que esperamos encontrar no ponto central da estrela, que são

$$
r_{0}=0.001 \mathrm{~km}, \quad M\left(r_{0}\right)=0, \quad P\left(r_{0}\right)=P_{c} .
$$

Para entendermos esses valores, começamos com o $r_{0}$. No referencial em que a origem das coordenadas se encontra no centro da estrela, o valor do raio em seu centro deve ser nulo; porém, não é possível realizar o cálculo tomando o valor de $r_{0}=0$. Assim, tomamos um valor de $r_{0}$ suficientemente próximo de zero. $M\left(r_{0}\right)$ é o valor da massa da estrela contida no interior de uma esfera de raio $r_{0}$. Como $r_{0}$ é (praticamente) nulo, e uma esfera de raio nulo é um ponto, esperamos que $M\left(r_{0}\right)=0 . P\left(r_{0}\right)$ é a pressão no centro da estrela. Aqui a pressão é máxima. Como podemos ver em (2.1.1), o valor da pressão diminui conforme aumentamos $r$. O valor de $P_{c}$ é desconhecido e diferente para cada estrela. Assim, resolvemos a TOV diversas vezes, cada vez com um valor diferente de $P_{c}$. Resolvendo a TOV com um único valor de $P_{c}$, obtemos, para a estrela descrita pela equação de estado utilizada e com a pressão central de valor $P_{c}$, o seu valor de massa e raio. Dessa forma, realizando esse processo repetidas vezes com diferentes valores de $P_{c}$, ao final obtemos uma tabela relacionando os valores de massa e raio possíveis. A partir dela, podemos fazer o gráfico de massa em função do raio, e compará-lo com os pontos observacionais conhecidos. Como um exemplo, temos o gráfico da figura 2.1, que é o mesmo presente na figura 3.4 para $B=57.5 \mathrm{MeV} / \mathrm{fm}^{3}$; porém, com os pontos não conectados. Em cada ponto, utilizamos uma pressão central diferente, sendo que em quatro deles, explicitamos o valor de $P_{c}$ na figura.

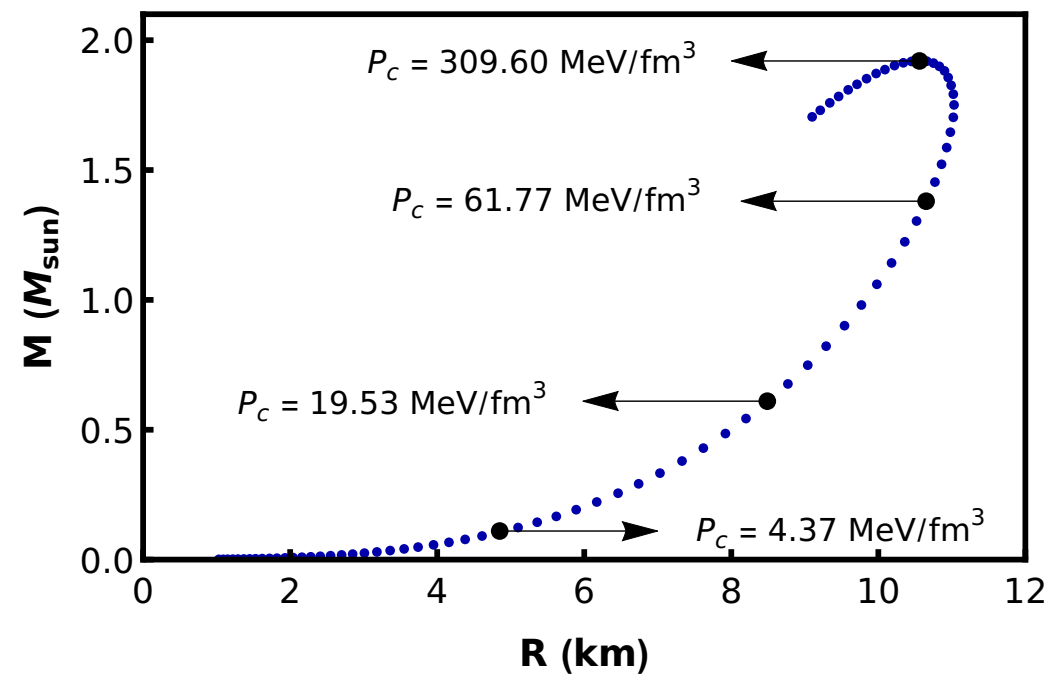

Figura 2.1: Pressão central utilizada em quatro pontos do diagrama de massa-raio. 
A resolução numérica das equações de TOV ocorre da seguinte forma: a partir das condições de contorno $\left(M\left(r_{0}\right)=0\right.$ e $\left.P\left(r_{0}\right)=P_{c}\right)$, é possível obter os valores de pressão $\left(P\left(r_{1}\right)\right)$ e de massa $\left(M\left(r_{1}\right)\right)$ no raio de valor

$$
r_{1}=r_{0}+\delta r
$$

onde fixamos o valor de $\delta r=0.001 \mathrm{~km}$ em todo o processo. Da mesma forma que conseguimos obter os valores de $P\left(r_{1}\right)$ e $M\left(r_{1}\right)$ a partir de $P\left(r_{0}\right)=P_{c}$ e $M\left(r_{0}\right)=0$, obtemos os valores de $P\left(r_{2}\right)$ e $M\left(r_{2}\right)$ a partir de $P\left(r_{1}\right)$ e $M\left(r_{1}\right)$, onde $r_{2}=r_{1}+\delta r$. Este procedimento continua até atingirmos a superfície da estrela, que é determinada quando chegamos em um valor de raio $r_{N}$ tal que a pressão neste raio é ${ }^{2}$

$$
P_{\text {sup }}=P\left(r_{N}\right)=0 \text {. }
$$

Desta forma, os valores de massa e raio total da estrela são

$$
M=M\left(r_{N}\right), \quad R=r_{N} .
$$

Uma explicação mais detalhada da resolução numérica das equações de TOV encontra-se no apêndice $B$, onde demonstramos o procedimento realizado para as equações de estado aqui utilizadas.

\subsection{Deformabilidade de Maré}

Um sistema binário de corpos compactos inevitavelmente perde energia através de ondas gravitacionais. Por outro lado, um corpo gera deformações no outro, e essas deformações alteram as ondas gravitacionais. Sendo assim, essas ondas nos trazem informações sobre o quanto um corpo deste sistema binário sofre deformação gerada pelo segundo corpo. A quantidade que pode ser medida experimentalmente através da detecção de ondas gravitacionais é a deformabilidade de maré, dada por

$$
\Lambda=\frac{2}{3} k_{2}(G C)^{-5}
$$

onde $C=M / R$ é a compacidade da estrela (massa sobre raio total) e $k_{2}$ é o número de deformabilidade de Love, que nos fornece uma medida da dificuldade de deformação de uma estrela diante de um campo de maré externo. Esse valor é dado pela equação

$$
\begin{aligned}
k_{2}= & \frac{8 C^{5}}{5}(1-2 C)^{2}[2+2 C(y-1)-y]\{2 C[6-3 y+3 C(5 y-8)] \\
& +4 C^{3}\left[13-11 y+C(3 y-2)+2 C^{2}(1+y)\right] \\
& \left.+3(1-2 C)^{2}[2-y+2 C(y-1)] \ln (1-2 C)\right\}^{-1},
\end{aligned}
$$

\footnotetext{
${ }^{2}$ Para o caso das estrelas híbridas deste trabalho, definimos a superfície da estrela no ponto em que a densidade bariônica atinge o valor zero. Este fato é melhor explicado no apêndice B.
} 
sendo

$$
y=\frac{R \beta(R)}{H(R)}-\frac{4 \pi R^{3} \epsilon_{\text {sup }}}{M}, \quad \beta(r)=\frac{d H(r)}{d r} .
$$

Uma dedução dessas equações é feita no apêndice C. O segundo termo de y é uma correção devido ao fato de que, no nosso modelo, a densidade de energia superficial $\epsilon_{\text {sup }} \equiv \epsilon(P=0)$ não é nula [39]. A função $H(r)$ está relacionada com a perturbação da métrica causada pelo segundo corpo do sistema binário, e deve satisfazer o sistema de equações diferenciais abaixo

$$
\begin{aligned}
\frac{d H}{d r}= & \beta(r) \\
\frac{d \beta}{d r}= & 2\left(1-\frac{2 G M(r)}{r}\right)^{-1} H(r)\left\{-2 \pi G\left[5 \epsilon(r)+9 P(r)+\frac{\epsilon(r)+P(r)}{d P / d \epsilon}\right]\right. \\
& \left.+\frac{3}{r^{2}}+2\left(1-\frac{2 G M(r)}{r}\right)^{-1}\left(\frac{G M(r)}{r^{2}}+4 \pi G r P(r)\right)^{2}\right\} \\
& +\frac{2 \beta(r)}{r}\left(1-\frac{2 G M(r)}{r}\right)^{-1}\left[-1+\frac{G M(r)}{r}+2 \pi G r^{2}(\epsilon(r)-P(r))\right] .
\end{aligned}
$$

Uma dedução deste sistema também é feita no apêndice C. Conhecendo as condições de contorno $[40,41]$

$$
H\left(r_{0}\right)=r_{0}^{2}, \quad \beta\left(r_{0}\right)=2 r_{0}, \quad r_{0} \approx 0,
$$

podemos resolver numericamente as equações (2.2.4) e (2.2.5). Como essas equações dependem dos valores de $P(r), \epsilon(r)$ e $M(r)$, a resolução numérica deve ser feita de forma simultânea com a resolução das equações de TOV. (Uma explicação mais detalhada da resolução numérica é feita no apêndice B). Uma vez que obtemos os valores de $H(R)$ e $\beta(R)$, podemos substituí-los na equação (2.2.3). Em seguida, substituindo o valor de $y$ na equação (2.2.2), obtemos o valor de $k_{2}$, e por fim, pela equação (2.2.1), obtemos o valor de $\Lambda$. 


\section{CApítulo}

\section{Estrelas de Quarks}

Como vimos no capítulo 1, uma das formas de modelar estrelas de nêutrons é supor que elas sejam constituídas inteiramente por matéria de quarks desconfinados. Este é o caso em que denominamos as estrelas de nêutrons por estrelas de quarks. Neste caso, usamos modelos da QCD que satisfaçam as propriedades conhecidas da teoria (como a liberdade assintótica e o confinamento de cor), tal como veremos na seção 3.1. Além disso, para que uma estrela de nêutrons contenha apenas matéria na fase desconfinada, a hipótese de Bodmer [42] - Witten [43] - Terazawa [44] deve ser satisfeita. Esta hipótese nos diz que a matéria estranha (formada pelos quarks up, down e strange desconfinados) é mais estável do que a matéria nuclear (quarks confinados em hádrons - como prótons e nêutrons). É importante ressaltar que essa hipótese não contradiz o fato da matéria ser mais abundante em seu estado nuclear. O que ocorre é que a matéria nuclear seria um meta-estado, cujo tempo médio de decaimento é de $10^{60}$ anos, superando a idade do Univeso, estimada em 13 bilhões de anos [42]. De forma matemática, a hipótese de Bodmer-Witten-Terazawa se traduz como

$$
\left.\frac{\epsilon}{\rho_{B}}\right|_{u, d, s} \leq\left.\frac{\epsilon}{\rho_{B}}\right|_{56 \mathrm{Fe}}=934 \mathrm{MeV}
$$

que implica que a matéria estranha é mais estável do que o isótopo ${ }^{56} \mathrm{Fe}$ (isótopo mais estável conhecido) ${ }^{1}$. Além disso, a matéria que compõe as estrelas de quarks também deve satisfazer o fato da matéria nuclear não decair espontaneamente em quarks up e down desconfinados (que chamamos de matéria de quarks). Assim, os modelos de quarks também devem satisfazer a relação

$$
\left.\frac{\epsilon}{\rho_{B}}\right|_{u, d} \geq\left.\frac{\epsilon}{\rho_{B}}\right|_{56 \mathrm{Fe}}=934 \mathrm{MeV},
$$

Isto significa que a matéria nuclear é mais estável do que a matéria de quarks.

${ }^{1} \epsilon / \rho_{B} \mid{ }_{56} \mathrm{Fe}=(930+4) \mathrm{MeV}$, onde o $+4 \mathrm{MeV}$ surge dos efeitos de superfícies do núcleo [45, 46]. 
Neste capítulo, estudaremos o modelo de sacola do MIT (seção 3.1) e as equações da MFTQCD (seção 3.2). Nestas seções, vamos apresentar os modelos e encontrar os valores dos parâmetros livres para os quais as equações (3.0.1) e (3.0.2) sejam satisfaeitas. Em seguida, obtemos os resultados das equações da TOV e a deformabilidade de maré. Por fim, comparamos os resultados com os dados experimentais e verificamos se o modelo analisado reproduz as informações experimentais disponíveis sobre estrelas de nêutrons.

\subsection{Modelo de Sacola do MIT}

Da QCD, sabemos que a intensidade das interações é inversamente proporcional à distância entre as partículas. Assim, quanto mais próximos dois quarks estiverem, menor será a interação entre eles. Este fato é conhecido como liberdade assintótica [47, 48] e é um resultado famoso obtido em teoria de perturbação da QCD. Ele implica que os quarks se comportam aproximadamente como partículas livres no interior dos hádrons, pois a distância entre eles é suficientemente pequena. A outra característica única da QCD é a hipótese do confinamento de cor. Ela nos diz que objetos que possuem carga de cor estarão sempre confinados em estados de carga de cor nula (singletos de cor). Objetos com carga de cor diferente de zero não podem se propagar como partículas livres [49]. De fato, apesar de termos diversas evidências experimentais da existência dos quarks, eles nunca foram detectados livremente [49].

O modelo de sacola do MIT é um dos modelos mais simples que temos. Apesar de sua simplicidade, ele incorpora as duas características da QCD mencionadas acima. Neste modelo, consideramos que os hádrons são como uma sacola em cujo interior encontramse os quarks confinados. Em seu exterior, há apenas o vácuo da QCD, responsável por exercer uma pressão sobre a sacola (pressão do vácuo, denotada por $B$ ). Os quarks, por sua vez, com seu movimento cinético, geram uma pressão que irá contrabalançar a pressão do vácuo [1]. Neste modelo, a pressão e a densidade de energia dos quarks deve levar em consideração, não apenas o seu movimento cinético, mas também a contribuição do vácuo da QCD. Com isso, as expressões de pressão, densidade de energia e densidade de número bariônico do modelo de sacola do MIT para o gás de quarks são dadas, respectivamente, por [8]

$$
\begin{aligned}
P_{\text {quarks }} & =\frac{1}{6 \pi^{2}} \sum_{q=u, d, s} g_{q} \int_{0}^{\infty} d k k^{3} \frac{\partial E_{q}}{\partial k}\left[f\left(k, \mu_{q}\right)+f\left(k,-\mu_{q}\right)\right]-B \\
\epsilon_{\text {quarks }} & =\frac{1}{2 \pi^{2}} \sum_{q=u, d, s} g_{q} \int_{0}^{\infty} d k k^{2} E_{q}\left[f\left(k, \mu_{q}\right)+f\left(k,-\mu_{q}\right)\right]+B
\end{aligned}
$$




$$
\rho_{B, \text { quarks }}=\frac{1}{3} \times \frac{1}{2 \pi^{2}} \sum_{q=u, d, s} g_{q} \int_{0}^{\infty} d k k^{2}\left[f\left(k, \mu_{q}\right)+f\left(k,-\mu_{q}\right)\right]
$$

onde descrevemos os quarks como um gás de Fermi. Nessas equações, temos que $\mu_{q}$ é o potencial químico; $E_{q}$ é a energia do quark q, que pode ser escrita como

$$
E_{q}=\sqrt{k_{q}^{2}+m_{q}^{2}}
$$

$g_{q}=2_{\text {spin }} \times 3_{\text {cor }}$ é a degenerescência do quark $q$; e $f$ é a função distribuição de Fermi

$$
f\left(k, \mu_{q}\right)=\frac{1}{\exp \left[\left(E_{k}-\mu_{q}\right) / T\right]+\theta_{q}},
$$

onde $\theta_{q}= \pm 1$ ( + , para férmions; - para bósons). O termo $1 / 3$ presente na densidade bariônica é devido ao fato da carga bariônica dos quarks ser $1 / 3$. A contribuição do gás de glúons é dada por [50]

$$
\begin{aligned}
P_{\text {glúons }} & =\frac{g_{g}}{6 \pi^{2}} \int_{0}^{\infty} d k k^{3} \frac{\partial E_{g}}{\partial k} f\left(k, \mu_{g}\right) ; \\
\epsilon_{\text {glúons }} & =\frac{g_{g}}{2 \pi^{2}} \int_{0}^{\infty} d k k^{2} E_{g} f\left(k, \mu_{q}\right) .
\end{aligned}
$$

Usando que $g_{g}=2_{\text {spin }} \times 8_{\text {cor }}$ e $m_{g}=0$ (e portanto, $E_{g}=k$ e $\partial E_{g} / \partial k=1$ ), obtemos as seguintes equações

$$
\begin{aligned}
P_{\text {glúons }} & =\frac{16}{6 \pi^{2}} \int_{0}^{\infty} d k k^{3} \frac{1}{\exp (k / T)-1}, \\
\epsilon_{\text {glúons }} & =\frac{16}{2 \pi^{2}} \int_{0}^{\infty} d k k^{3} \frac{1}{\exp (k / T)-1},
\end{aligned}
$$

cujas integrais podem ser calculadas analiticamente, resultando em

$$
\begin{aligned}
P_{\text {glúons }} & =\frac{8 \pi^{2}}{45} T^{4}, \\
\epsilon_{\text {glúons }} & =\frac{8 \pi^{2}}{15} T^{4} .
\end{aligned}
$$

Além disso, iremos considerar que há elétrons na fase de quarks da estrela de nêutrons. A contribuição do gás de elétrons é dada por

$$
\begin{aligned}
P_{e} & =\frac{g_{e}}{6 \pi^{2}} \int_{0}^{\infty} d k k^{3} \frac{\partial E_{e}}{\partial k}\left[f\left(k, \mu_{e}\right)+f\left(k,-\mu_{e}\right)\right] ; \\
\epsilon_{e} & =\frac{g_{e}}{2 \pi^{2}} \int_{0}^{\infty} d k k^{2} E_{e}\left[f\left(k, \mu_{e}\right)+f\left(k,-\mu_{e}\right)\right],
\end{aligned}
$$


onde $g_{e}=2_{\text {spin }}$. Considerando todas as componentes do gás, a pressão, a densidade de energia e a densidade bariônica da fase de quarks são dadas por

$$
\begin{aligned}
P_{Q}= & \frac{8 \pi^{2}}{45} T^{4}+\frac{1}{6 \pi^{2}} \sum_{q} g_{q} \int_{0}^{\infty} d k k^{3} \frac{\partial E_{q}}{\partial k}\left[f\left(k, \mu_{q}\right)+f\left(k,-\mu_{q}\right)\right] \\
& +\frac{g_{e}}{6 \pi^{2}} \int_{0}^{\infty} d k k^{3} \frac{\partial E_{e}}{\partial k}\left[f\left(k, \mu_{e}\right)+f\left(k,-\mu_{e}\right)\right]-B ; \\
\epsilon_{Q}= & \frac{8 \pi^{2}}{15} T^{4}+\frac{1}{2 \pi^{2}} \sum_{q} g_{q} \int_{0}^{\infty} d k k^{2} E_{q}\left[f\left(k, \mu_{q}\right)+f\left(k,-\mu_{q}\right)\right] \\
& +\frac{g_{e}}{2 \pi^{2}} \int_{0}^{\infty} d k k^{2} E_{e}\left[f\left(k, \mu_{e}\right)+f\left(k,-\mu_{e}\right)\right]+B \\
\rho_{B, Q}= & \frac{1}{3} \frac{1}{2 \pi^{2}} \sum_{q} g_{q} \int_{0}^{\infty} d k k^{2}\left[f\left(k, \mu_{q}\right)+f\left(k,-\mu_{q}\right)\right]
\end{aligned}
$$

onde os glúons e os elétrons não contribuem para a densidade bariônica (pois ambos tem carga bariônica nula).

Como iremos aplicar essas equações de estado em estrelas de nêutrons, que têm temperaturas relativamente baixas, podemos tomar o limite $T \rightarrow 0$. Neste limite, a distribuição de Fermi pode ser escrita como

$$
\begin{aligned}
\lim _{T \rightarrow 0} f\left(k, \mu_{q}\right) & =\Theta\left(\mu_{q}-E_{q}\right) ; \\
\lim _{T \rightarrow 0} f\left(k,-\mu_{q}\right) & =0,
\end{aligned}
$$

onde $\Theta$ é a função de Heaviside, definida como

$$
\Theta\left(\mu_{q}-E_{q}\right)=\left\{\begin{array}{l}
0, \mu_{q}-E_{q}<0 \\
1, \mu_{q}-E_{q}>0 .
\end{array}\right.
$$

Com isso, conseguimos calcular as integrais presentes nas equações de estado analiticamente. Por exemplo, para calcular a pressão gerada pelo quark up, teremos

$$
\begin{aligned}
P_{u} & =\frac{1}{6 \pi^{2}} g_{u} \int_{0}^{\infty} d k k^{3} \frac{\partial E_{u}}{\partial k}\left[f\left(k, \mu_{u}\right)+f\left(k,-\mu_{u}\right)\right] \\
& =\frac{1}{6 \pi^{2}} 6 \int_{0}^{\infty} d k k^{3} \frac{\partial}{\partial k}\left(\sqrt{k_{u}^{2}+m_{u}^{2}}\right)\left[\Theta\left(\mu_{u}-E_{u}\right)+0\right] \\
& =\frac{1}{\pi^{2}} \int_{0}^{\infty} d k_{u} \frac{k_{u}^{4}}{\sqrt{k_{u}^{2}+m_{u}^{2}}} \Theta\left(\mu_{u}-E_{u}\right) .
\end{aligned}
$$

Pela definição da função de Heaviside, apenas os integrandos da função acima que satisfazem

$$
\mu_{u}>\sqrt{k_{u}^{2}+m_{u}^{2}} \Rightarrow k_{u}<\sqrt{\mu_{u}^{2}-m_{u}^{2}}
$$


são diferentes de zero. Assim, a pressão dos quarks up pode ser escrita como

$$
P_{u}=\frac{1}{\pi^{2}} \int_{0}^{k_{F, u}} d k_{u} \frac{k_{u}^{4}}{\sqrt{k_{u}^{2}+m_{u}^{2}}},
$$

onde $k_{F, u}=\sqrt{\mu_{F, u}^{2}-m_{u}^{2}}$ e $\mu_{F, u}=E_{u}$, conhecidos como o momento de Fermi e potencial químico de Fermi do quark up, respectivamente. Podemos resolver essa integral analiticamente e obter

$$
P_{u}=\frac{1}{4 \pi^{2}}\left[\mu_{F, u} k_{F, u}\left(\mu_{F, u}^{2}-\frac{5}{2} m_{u}^{2}\right)+\frac{3}{2} m_{q}^{4} \ln \left(\frac{\mu_{F, u}+k_{F, u}}{m_{u}}\right)\right] .
$$

Analogamente para os demais termos e demais equações, podemos obter que

$$
\begin{aligned}
P_{Q}= & \frac{1}{4 \pi^{2}} \sum_{q=u, d, s}\left[\mu_{F, q} k_{F, q}\left(\mu_{F, q}^{2}-\frac{5}{2} m_{q}^{2}\right)+\frac{3}{2} m_{q}^{4} \ln \left(\frac{\mu_{F, q}+k_{F, q}}{m_{q}}\right)\right] \\
& +\frac{1}{12 \pi^{2}}\left[\mu_{F, e} k_{F, e}\left(\mu_{F, e}^{2}-\frac{5}{2} m_{e}^{2}\right)+\frac{3}{2} m_{e}^{4} \ln \left(\frac{\mu_{F, e}+k_{F, e}}{m_{e}}\right)\right]-B ; \\
\epsilon_{Q}= & \frac{3}{4 \pi^{2}} \sum_{q=u, d, s}\left[\mu_{F, q} k_{F, q}\left(\mu_{F, q}^{2}-\frac{1}{2} m_{q}^{2}\right)-\frac{1}{2} m_{q}^{4} \ln \left(\frac{\mu_{F, q}+k_{F, q}}{m_{q}}\right)\right] \\
& +\frac{1}{4 \pi^{2}}\left[\mu_{F, e} k_{F, e}\left(\mu_{F, e}^{2}-\frac{1}{2} m_{e}^{2}\right)-\frac{1}{2} m_{e}^{4} \ln \left(\frac{\mu_{F, e}+k_{F, e}}{m_{e}}\right)\right]+B ; \\
\rho_{B}= & \frac{1}{3 \pi^{2}} \sum_{q=u, d, s} k_{F, q}^{3},
\end{aligned}
$$

sendo $\mu_{F, q}$ e $\mu_{F, e}$ o potencial químico de Fermi dos quarks e elétrons, dado por

$$
\mu_{F, q}=\sqrt{k_{F, q}^{2}+m_{q}^{2}}, \quad \mu_{F, e}=\sqrt{k_{F, e}^{2}+m_{e}^{2}},
$$

e $k_{F, q}$ e $k_{F, e}$ os momentos de Fermi.

Para calcular a pressão e a densidade de energia para um dado valor de densidade bariônica, precisamos antes encontrar os valores dos momentos de Fermi. Temos quatro momentos de Fermi, e apenas uma equação que os relaciona com a densidade bariônica (equação (3.1.8)); ou seja, temos quatro variáveis e apenas uma equação. Precisamos de mais três equações para encontrar os valores de $k_{F, q}$ e $k_{F, e}$ para um dado valor de $\rho_{B}$. Uma delas é a condição de neutralidade elétrica, dada por

$$
\sum_{q} q_{q} \frac{k_{F, q}^{3}}{\pi^{2}}-\frac{k_{F, e}^{3}}{3 \pi^{2}}=0,
$$

onde $q_{q}$ é o valor da carga elétrica do quark $q$, fornecido na tabela 3.1. Além disso, podemos também considerar as possíveis transformações entre os quarks e o elétron que 


\begin{tabular}{|ccc|}
\hline Partícula & Massa & Carga Elétrica \\
\hline \hline $\mathrm{u}$ & $5 \mathrm{MeV}$ & $+2 / 3$ \\
\hline $\mathrm{d}$ & $7 \mathrm{MeV}$ & $-1 / 3$ \\
\hline $\mathrm{s}$ & $100 \mathrm{MeV}$ & $-1 / 3$ \\
\hline $\mathrm{e}^{-}$ & $0.5 \mathrm{MeV}$ & -1 \\
\hline
\end{tabular}

Tabela 3.1: Massa e carga elétrica das partículas presentes na fase de quarks.

satisfazem a conservação da carga bariônica e elétrica

$$
\begin{gathered}
d \rightarrow u+e+\bar{\nu}_{e}, \quad s \rightarrow u+e+\bar{\nu}_{e} \\
u+e \rightarrow d+\nu_{e}, \quad u+e \rightarrow s+\nu_{e}, \\
s+u \leftrightarrow d+u .
\end{gathered}
$$

Com essas transformações, usamos a equação

$$
\sum_{i} X_{i} \mu_{i}=0
$$

onde $X_{i}$ é o coeficiente da partícula $i$ (por exemplo, para a reação $d \rightarrow u+e$, teremos $X_{d}=-X_{u}=-X_{e}=1$ ). Dessa forma, obtemos as relações

$$
\begin{aligned}
& \mu_{F, d}=\mu_{F, u}+\mu_{F, e}, \\
& \mu_{F, s}=\mu_{F, d},
\end{aligned}
$$

e com isso, obtemos as relações entre os momentos de Fermi. Vemos que, para um dado valor de densidade bariônica $\rho_{B}$, conseguimos resolver numericamente o sistema formado pelas equações (3.1.8), (3.1.10), (3.1.12) e (3.1.13), reescritas, respectivamente, abaixo

$$
\left\{\begin{array}{l}
\rho_{B}=\frac{1}{3 \pi^{2}}\left(k_{F, u}^{3}+k_{F, d}^{3}+k_{F, s}^{3}\right) \\
0=2 k_{F, u}^{3}-k_{F, d}^{3}-k_{F, s}^{3}-k_{F, e}^{3} \\
\sqrt{k_{F, d}^{2}+m_{d}^{2}}=\sqrt{k_{F, u}^{2}+m_{u}^{2}}+\sqrt{k_{F, e}^{2}+m_{e}^{2}} \\
k_{F, d}^{2}+m_{d}^{2}=k_{F, s}^{2}+m_{s}^{2} .
\end{array}\right.
$$

Uma vez que encontramos os valores dos momentos de Fermi para um dado valor de $\rho_{B}$, é fácil obter os seus respectivos potenciais químicos de Fermi através da equação (3.1.9). Em seguida, podemos substituir estes valores encontrados nas equações (3.1.6) e (3.1.7) para obter os valores de pressão e energia para o dado valor de densidade bariônica. Repetindo esse procedimento para vários valores fixos de $\rho_{B}$, podemos construir os gráficos apresentados nas figuras 3.1 e 3.2 . 

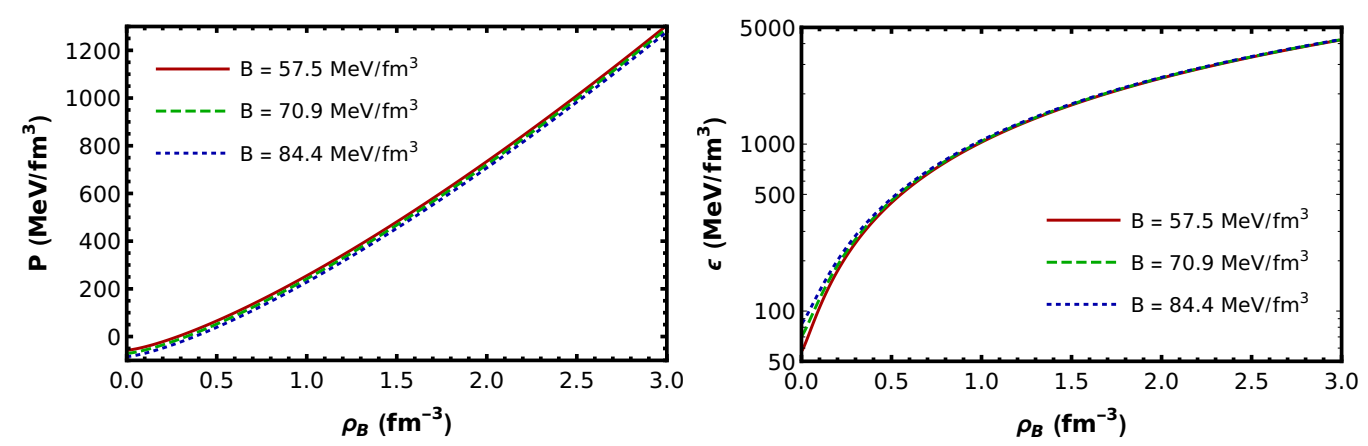

Figura 3.1: Pressão (à esquerda) e densidade de energia (à direita) em função da densidade bariônica do modelo de sacola do MIT.

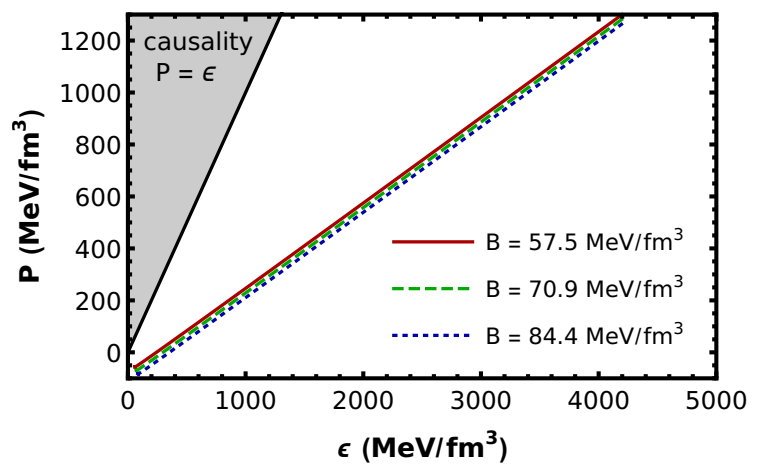

Figura 3.2: Pressão em função da densidade de energia do modelo de sacola do MIT.

Numa estrela de quarks, a hipótese de Bodmer-Witten-Terazawa deve ser satisfeita. Vamos verificar como isto acontece. Para um dado valor de $B$, resolvemos numericamente o sistema de equações (3.1.14) juntamente com a equação (3.1.6) de forma que os momentos de Fermi resultem em uma pressão nula (pressão na superfície da sacola). Uma vez que encontramos os valores dos momentos de Fermi, podemos calcular a densidade bariônica e a densidade de energia. Assim, é possível encontrar o valor de $\epsilon /\left.\rho_{B}\right|_{q=u, d, s}$. Em seguida, repetimos o mesmo procedimento, porém desconsiderando o quark strange. Resolvemos numericamente o sistema de equações

$$
\left\{\begin{array}{l}
\rho_{B}=\frac{1}{3 \pi^{2}}\left(k_{F, u}^{3}+k_{F, d}^{3}\right) \\
0=2 k_{F, u}^{3}-k_{F, d}^{3}-k_{F, e}^{3} \\
\sqrt{k_{F, d}^{2}+m_{d}^{2}}=\sqrt{k_{F, u}^{2}+m_{u}^{2}}+\sqrt{k_{F, e}^{2}+m_{e}^{2}}
\end{array}\right.
$$

juntamente com a equação (3.1.6) (porém, sem a contribuição do quark strange) de forma que a pressão seja nula. Encontrando os valores dos momentos de Fermi, calculamos a densidade bariônica e a densidade de energia, e por fim, dividimos este último pelo anterior, obtendo o valor de $\epsilon /\left.\rho_{B}\right|_{q=u, d}$. Repetindo esse procedimento para diferentes valores de $B$, podemos fazer o gráfico apresentado na figura 3.3. Com isso, podemos ver 


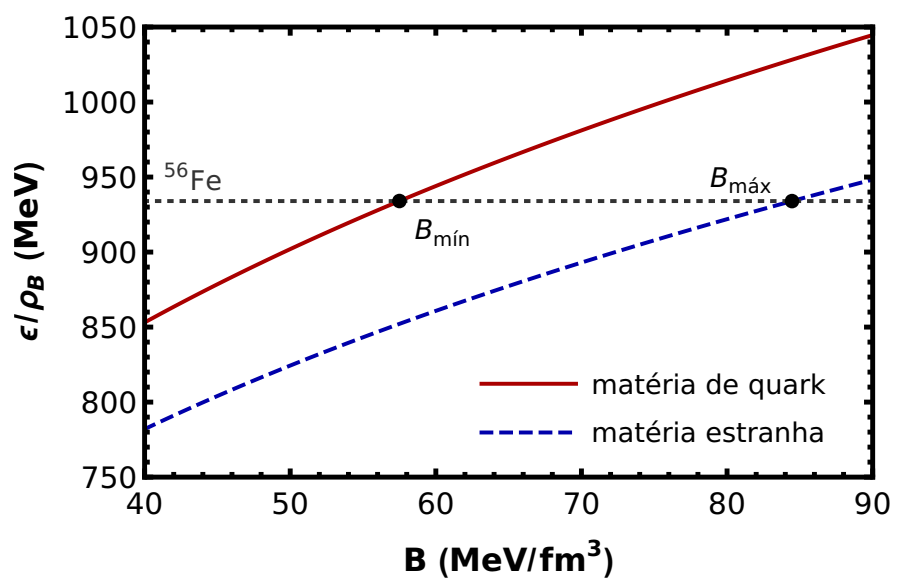

Figura 3.3: Valores de $\epsilon / \rho_{B}$ em função de $B$ para a matéria de quark (em vermelho) e para a matéria estranha (em azul). Em cinza, temos $\epsilon /\left.\rho_{B}\right|_{56} \mathrm{Fe}=934 \mathrm{MeV}$.

que a condição

$$
\left.\frac{\epsilon}{\rho_{B}}\right|_{q=u, d} \geq\left.\frac{\epsilon}{\rho_{B}}\right|_{56 \mathrm{Fe}}=934 \mathrm{MeV},
$$

implica em um valor mínimo de $B: B_{\text {mín }}=57.5 \mathrm{MeV} / \mathrm{fm}^{3}$. A condição

$$
\left.\frac{\epsilon}{\rho_{B}}\right|_{q=u, d, s} \leq\left.\frac{\epsilon}{\rho_{B}}\right|_{56 \mathrm{Fe}}=934 \mathrm{MeV}
$$

implica em um valor máximo de $B: B_{\text {máx }}=84.4 \mathrm{MeV} / \mathrm{fm}^{3}$. Juntando estes limites, $B$ deve ser tal que

$$
57.5 \mathrm{MeV} / \mathrm{fm}^{3}<B<84.4 \mathrm{MeV} / \mathrm{fm}^{3} .
$$

Conhecendo os possíveis valores de $B$, escolhemos os valores

$$
B=57.5,70.9,84.4 \mathrm{MeV} / \mathrm{fm}^{3}
$$

e obtemos as suas respectivas equações de estado (figuras 3.1 e 3.2). Em seguida, resolvemos as equações de TOV juntamente com as quantidades necessárias para se obter a deformabilidade de maré e encontramos os gráficos apresentados na figura 3.4.

Apesar dos valores de deformabilidade de maré estarem de acordo com o dado experimental (figura 3.4, à direita), vemos que o mesmo não acontece para o diagrama de massa-raio (figura 3.4, à esquerda), já que obtemos curvas com massa e raio muito pequenos em relação aos dados experimentais. Entretanto, apesar do modelo de sacola do MIT não ser um bom modelo para o QGP frio, ele é um ótimo modelo de estudo. Como a única variável livre é a constante de sacola $(B)$, vemos que um aumento na pressão (originado pela diminuição do valor de $B$ ) resulta em uma curva de massa-raio com valores maiores de massa e raio, além de aumentar também o valor da deformabilidade de maré. Desta 

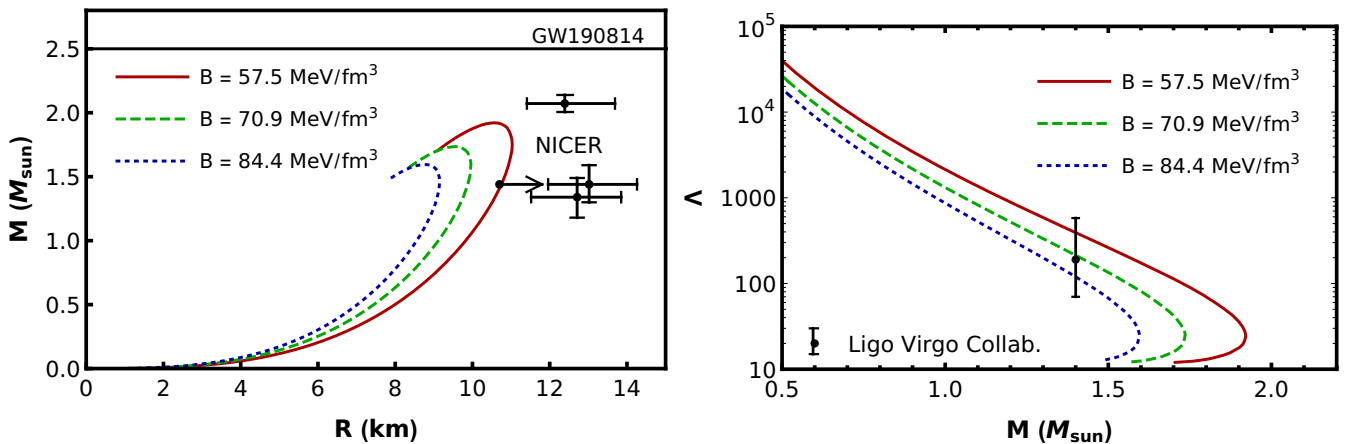

Figura 3.4: Relação massa-raio à esquerda e deformabilidade de maré à direita para uma estrela de quarks cujos constituintes são descritos pelo modelo de sacola do MIT.

forma, concluímos que, para termos resultados mais próximos dos dados experimentais, é necessário um modelo que tenha uma pressão maior do que a gerada pelo modelo de sacola do MIT, como veremos na próxima seção.

\subsection{QCD em Campo Médio (MFTQCD)}

\subsubsection{Lagrangiana da MFTQCD}

A lagrangiana da QCD é dada por

$$
\mathcal{L}_{\mathrm{QCD}}=-\frac{1}{4} F_{\mu \nu}^{a} F^{a \mu \nu}+\sum_{q=1}^{N_{f}} \bar{\psi}_{i}^{q}\left[i \gamma^{\mu}\left(\delta_{i j} \partial_{\mu}-i g T_{i j}^{a} G_{\mu}^{a}\right)-\delta_{i j} m_{q}\right] \psi_{j}^{q},
$$

sendo $q$ o sabor dos quarks ( $q=1,2,3$, referente aos quarks up, down e strange); $i$ e $j$, os índices de cor do quark ( $i, j=1,2,3$, referentes às cores red, green e blue); $a$, o índice de cor do glúon $(a=1, \ldots, 8) ; g$, a constante de acoplamento do $\mathrm{SU}(3) ; \boldsymbol{T}_{a}=\boldsymbol{\lambda}_{a} / 2$, com $\lambda_{a}$ sendo os geradores do grupo SU(3), escritas explicitamente no apêndice D; e

$$
F^{a \mu \nu}=\partial^{\mu} G^{a \nu}-\partial^{\nu} G^{a \mu}+g f^{a b c} G^{b \mu} G^{c \nu},
$$

onde $G_{\mu}^{a}$ é o campo dos glúons, $\psi$ é o campo dos quarks e $f^{a b c}$ são as constantes de estrutura do grupo SU(3).

O campo dos glúons no espaço de momentos pode ser escrito como uma soma [36, 12, 51, 52]:

$$
\tilde{G}^{a \mu}(k)=\tilde{A}^{a \mu}(k)+\tilde{\alpha}^{a \mu}(k),
$$

onde $\tilde{A}^{a \mu}$ é a componente de baixo momento (soft) do campo dos gluons e $\tilde{\alpha}^{a \mu}$ é a componente de alto momento (hard) do campo dos gluons. Neste ponto seria necessário definir um momento de corte, que separa os dois regimes. Ele poderia ser $k_{0} \simeq 1-2 \mathrm{GeV}$. Ou 
poderia ser a escala $\Lambda_{Q C D} \simeq 200 \mathrm{MeV}$. Mas, para o que vamos fazer a seguir, basta dizer que baixo momento significa momento próximo de zero.

A seguir, substituimos a equação (3.2.3) na equação (3.2.2), obtendo

$$
F^{a \mu \nu}=\partial^{\mu}\left(A^{a \nu}+\alpha^{a \nu}\right)-\partial^{\nu}\left(A^{a \mu}+\alpha^{a \mu}\right)+g f^{a b c}\left(A^{b \mu}+\alpha^{b \mu}\right)\left(A^{c \nu}+\alpha^{c \nu}\right) .
$$

que será usada mais adiante. Feita a decomposição do campo de glúons, vamos fazer uma aproximação para os soft glúons e, em seguida, outra para os hard glúons.

\subsubsection{Aproximação para os Soft Glúons}

O campo $A^{a \mu}(x)$ (no espaço das configurações) pode ser escrito como a transformada de Fourier do campo $\tilde{A}^{a \mu}(k)$ (no espaço dos momentos):

$$
A^{a \mu}(x) \propto \int d^{4} k e^{i k \cdot x} \tilde{A}^{a \mu}(k) .
$$

Aplicando a derivada $\partial^{\nu}$ em ambos os lados da equação, encontramos

$$
\partial^{\nu} A^{a \mu}(x) \propto \int d^{4} k i k^{\nu} e^{i k \cdot x} \tilde{A}^{a \mu}(k) .
$$

Os momentos de $A^{a \mu}$ são pequenos e podemos fazer a aproximação $k^{\nu} \approx 0$. Como consequência, obtemos $\partial^{\nu} A^{a \mu}(x)=0$. Usando essa aproximação, a equação (3.2.4) pode ser escrita como

$$
F^{a \mu \nu}=\partial^{\mu} \alpha^{a \nu}-\partial^{\nu} \alpha^{a \mu}+g f^{a b c}\left(A^{b \mu} A^{c \nu}+A^{b \mu} \alpha^{c \nu}+\alpha^{b \mu} A^{c \nu}+\alpha^{b \mu} \alpha^{c \nu}\right) .
$$

Definindo os termos independentes de $A^{a \mu}$ como

$$
\Gamma^{a \mu \nu}=\partial^{\mu} \alpha^{a \nu}-\partial^{\nu} \alpha^{a \nu}+g f^{a b c} \alpha^{b \mu} \alpha^{c \nu}
$$

a equação acima se transforma em

$$
F^{a \mu \nu}=\Gamma^{a \mu \nu}+g f^{a b c} A^{b \mu} A^{c \nu}+g f^{a b c} A^{b \mu} \alpha^{c \nu}+g f^{a b c} \alpha^{b \mu} A^{c \nu} .
$$

Vamos agora substituir $F^{a \mu \nu}$ na equação (3.2.1). Primeiro observamos que

$$
\begin{aligned}
F_{\mu \nu}^{a} F^{a \mu \nu}= & {\left[\Gamma_{\mu \nu}^{a}+g f^{a b c}\left(A_{\mu}^{b} A_{\nu}^{c}+A_{\mu}^{b} \alpha_{\nu}^{c}+\alpha_{\mu}^{b} A_{\nu}^{c}\right)\right] \times } \\
& {\left[\Gamma^{a \mu \nu}+g f^{a d e}\left(A^{d \mu} A^{e \nu}+A^{d \mu} \alpha^{e \nu}+\alpha^{d \mu} A^{e \nu}\right)\right] } \\
= & \Gamma_{\mu \nu}^{a} \Gamma^{a \mu \nu}+2 g f^{a b c} \Gamma_{\mu \nu}^{a}\left(A_{\mu}^{b} A_{\nu}^{c}+A_{\mu}^{b} \alpha_{\nu}^{c}+\alpha_{\mu}^{b} A_{\nu}^{c}\right) \\
+ & g^{2} f^{a b c} f^{a d e}\left(A_{\mu}^{b} A_{\nu}^{c}+A_{\mu}^{b} \alpha_{\nu}^{c}+\alpha_{\mu}^{b} A_{\nu}^{c}\right)\left(A^{d \mu} A^{e \nu}+A^{d \mu} \alpha^{e \nu}+\alpha^{d \mu} A^{e \nu}\right) .
\end{aligned}
$$


Substituindo este resultado na equação (3.2.1), encontramos

$$
\begin{aligned}
\mathcal{L}_{\mathrm{QCD}}= & -\frac{1}{4} \Gamma_{\mu \nu}^{a} \Gamma^{a \mu \nu}-\frac{g}{2} f^{a b c} \Gamma_{\mu \nu}^{a}\left(A_{\mu}^{b} A_{\nu}^{c}+A_{\mu}^{b} \alpha_{\nu}^{c}+\alpha_{\mu}^{b} A_{\nu}^{c}\right) \\
& -\frac{g^{2}}{4} f^{a b c} f^{a d e}\left(A_{\mu}^{b} A_{\nu}^{c}+A_{\mu}^{b} \alpha_{\nu}^{c}+\alpha_{\mu}^{b} A_{\nu}^{c}\right)\left(A^{d \mu} A^{e \nu}+A^{d \mu} \alpha^{e \nu}+\alpha^{d \mu} A^{e \nu}\right) \\
& +\sum_{q=1}^{N_{f}} \bar{\psi}_{i}^{q}\left\{i \gamma^{\mu}\left[\delta_{i j} \partial_{\mu}-i g T_{i j}^{a}\left(A_{\mu}^{a}+\alpha_{\mu}^{a}\right)\right]-\delta_{i j} m_{q}\right\} \psi_{j}^{q} .
\end{aligned}
$$

Agora vamos aproximar o campo dos soft gluons pelo seu valor esperado no vácuo:

$$
\begin{aligned}
& A^{a \mu} \rightarrow\left\langle A^{a \mu}\right\rangle, \\
& A^{a \mu} A^{b \nu} \rightarrow\left\langle A^{a \mu} A^{b \nu}\right\rangle, \\
& A^{a \mu} A^{b \nu} A^{c \rho} \rightarrow\left\langle A^{a \mu} A^{b \nu} A^{c \rho}\right\rangle, \\
& A^{a \mu} A^{b \nu} A^{c \rho} A^{d \eta} \rightarrow\left\langle A^{a \mu} A^{b \nu} A^{c \rho} A^{d \eta}\right\rangle .
\end{aligned}
$$

Como não há uma direção privilegiada nem no espaço das configurações e nem no espaço de cor, as componentes com número ímpar de campos devem se anular:

$$
\begin{aligned}
& \left\langle A^{a \mu}\right\rangle=0, \\
& \left\langle A^{a \mu} A^{b \nu} A^{c \rho}\right\rangle=0 .
\end{aligned}
$$

As componentes com número par de campos sobrevivem e os seus valores esperados no vácuo podem ser parametrizados em termos de escalas de energia [53, 54]:

$$
\begin{aligned}
\left\langle A^{a \mu} A^{b \nu}\right\rangle & =-\frac{\delta^{a b}}{8} \frac{g^{\mu \nu}}{4} \mu_{0}^{2}, \\
\left\langle A_{\mu}^{a} A_{\nu}^{b} A^{c \rho} A^{d \eta}\right\rangle & =\frac{\phi_{0}^{4}}{(32)(34)}\left[g_{\mu \nu} g^{\rho \eta} \delta^{a b} \delta^{c d}+g_{\mu}^{\rho} g_{\nu}^{\eta} \delta^{a c} \delta^{b d}+g_{\mu}^{\eta} g_{\nu}^{\rho} \delta^{a d} \delta^{b c}\right],
\end{aligned}
$$

onde $\mu_{0}$ e $\phi_{0}$ são as escalas de energia que serão determinadas com o auxílio de informações experimentais. Substituindo estas aproximações na lagrangiana da QCD, encontramos:

$$
\begin{aligned}
\mathcal{L}_{\mathrm{QCD}}= & -\frac{1}{4} \Gamma_{\mu \nu}^{a} \Gamma^{a \mu \nu}-\frac{g}{2} f^{a b c} \Gamma_{\mu \nu}^{a}\left\langle A_{\mu}^{b} A_{\nu}^{c}\right\rangle-\frac{g^{2}}{4} f^{a b c} f^{a d e}\left(\left\langle A_{\mu}^{b} A_{\nu}^{c} A^{d \mu} A^{e \nu}\right\rangle\right. \\
& \left.+\alpha^{e \nu} \alpha_{\nu}^{c}\left\langle A_{\mu}^{b} A^{d \mu}\right\rangle+\alpha_{\nu}^{c} \alpha^{d \mu}\left\langle A_{\mu}^{b} A^{e \nu}\right\rangle+\alpha_{\mu}^{b} \alpha^{e \nu}\left\langle A_{\nu}^{c} A^{d \mu}\right\rangle+\alpha_{\mu}^{b} \alpha^{d \mu}\left\langle A_{\nu}^{c} A^{e \nu}\right\rangle\right) \\
& +\sum_{q=1}^{N_{f}} \bar{\psi}_{i}^{q}\left\{i \gamma^{\mu}\left[\delta_{i j} \partial_{\mu}-i g T_{i j}^{a} \alpha_{\mu}^{a}\right]-\delta_{i j} m_{q}\right\} \psi_{j}^{q}
\end{aligned}
$$


onde os termos com potência ímpar de $A_{\mu}$ foram desprezados. Na equação acima, o segundo termo do lado direito é nulo. De fato:

$$
\begin{aligned}
-\frac{g}{2} f^{a b c} \Gamma_{\mu \nu}^{a}\left\langle A_{\mu}^{b} A_{\nu}^{c}\right\rangle & =-\frac{g}{2} f^{a b c} \Gamma_{\mu \nu}^{a} g_{\alpha \mu} g_{\beta \nu}\left\langle A^{b \alpha} A^{c \beta}\right\rangle \\
& =-\frac{g}{2} f^{a b c} \Gamma_{\mu \nu}^{a} g_{\alpha \mu} g_{\beta \nu}\left(\frac{\delta^{b c}}{8} \frac{g^{\alpha \beta}}{4} \mu_{0}^{2}\right) \\
& =-\frac{g}{2} f^{a b b} \Gamma_{\mu \nu}^{a} g_{\mu \nu}\left(\frac{1}{8}\right)\left(\frac{1}{4}\right) \mu_{0}^{2},
\end{aligned}
$$

Como $f^{a b c}$ é antissimétrico, segue que $f^{a b b}=0$ e assim:

$$
-\frac{g}{2} f^{a b c} \Gamma_{\mu \nu}^{a}\left\langle A_{\mu}^{b} A_{\nu}^{c}\right\rangle=0
$$

Os termos da segunda linha de (3.2.10) são do tipo $\alpha^{2}\left\langle A^{2}\right\rangle$ e vão resultar num termo de massa para os hard gluons, que é gerada pela interação com os soft gluons. Para deixar isso mais claro, vamos reescrever estes termos. Vamos defini-los como:

$$
\begin{aligned}
A_{2} \equiv & -\frac{g^{2}}{4} f^{a b c} f^{a d e}\left(\alpha^{e \nu} \alpha_{\nu}^{c}\left\langle A_{\mu}^{b} A^{d \mu}\right\rangle\right. \\
& \left.+\alpha_{\nu}^{c} \alpha^{d \mu}\left\langle A_{\mu}^{b} A^{e \nu}\right\rangle+\alpha_{\mu}^{b} \alpha^{e \nu}\left\langle A_{\nu}^{c} A^{d \mu}\right\rangle+\alpha_{\mu}^{b} \alpha^{d \mu}\left\langle A_{\nu}^{c} A^{e \nu}\right\rangle\right),
\end{aligned}
$$

Substituindo a equação (3.2.8) em $A_{2}$, obtemos

$$
\begin{aligned}
A_{2} & =-\frac{\mu_{0}^{2}}{32} \frac{g^{2}}{4} f^{a b c} f^{a d e}\left(\alpha^{e \nu} \alpha_{\nu}^{c} \delta^{b d} \delta_{\mu}^{\mu}+\alpha_{\nu}^{c} \alpha^{d \mu} \delta^{b e} \delta_{\mu}^{\nu}+\alpha_{\mu}^{b} \alpha^{e \nu} \delta^{c d} \delta_{\nu}^{\mu}+\alpha_{\mu}^{b} \alpha^{d \mu} \delta^{c e} \delta_{\nu}^{\nu}\right) \\
& =-\frac{\mu_{0}^{2}}{32} \frac{g^{2}}{4} f^{a b c} f^{a d e}\left(4 \alpha^{e \nu} \alpha_{\nu}^{c} \delta^{b d}+\alpha_{\mu}^{c} \alpha^{d \mu} \delta^{b e}+\alpha_{\mu}^{b} \alpha^{e \mu} \delta^{c d}+4 \alpha_{\mu}^{b} \alpha^{d \mu} \delta^{c e}\right) \\
& =-\frac{\mu_{0}^{2}}{32} \frac{g^{2}}{4}\left(4 f^{a b c} f^{a b e} \alpha^{e \nu} \alpha_{\nu}^{c}+f^{a b c} f^{a d b} \alpha_{\mu}^{c} \alpha^{d \mu}+f^{a b c} f^{a c e} \alpha_{\mu}^{b} \alpha^{e \mu}+4 f^{a b c} f^{a d c} \alpha_{\mu}^{b} \alpha^{d \mu}\right) .
\end{aligned}
$$

Usando a seguinte propriedade

$$
f^{a c d} f^{b c d}=3 \delta^{a b}
$$

e o fato das constantes de estrutura de SU(3) serem totalmente antissimétricas, obtemos

$$
\begin{aligned}
A_{2} & =-3 \frac{\mu_{0}^{2}}{32} \frac{g^{2}}{4}\left(4 \delta^{c e} \alpha^{e \nu} \alpha_{\nu}^{c}-\delta^{c d} \alpha_{\mu}^{c} \alpha^{d \mu}-\delta^{b e} \alpha_{\mu}^{b} \alpha^{e \mu}+4 \delta^{b d} \alpha_{\mu}^{b} \alpha^{d \mu}\right) \\
& =-3 \frac{\mu_{0}^{2}}{32} \frac{g^{2}}{4}\left(4 \alpha^{c \nu} \alpha_{\nu}^{c}-\alpha_{\mu}^{c} \alpha^{c \mu}-\alpha_{\mu}^{b} \alpha^{b \mu}+4 \alpha_{\mu}^{b} \alpha^{b \mu}\right) .
\end{aligned}
$$

E como os índices são mudos, chegamos a:

$$
A_{2}=-\frac{9}{4} \frac{1}{16} g^{2} \mu_{0}^{2} \alpha_{\mu}^{b} \alpha^{b \mu} .
$$


Calculando separadamente o termo proporcional a $\left\langle A_{\mu}^{b} A_{\nu}^{c} A^{d \mu} A^{e \nu}\right\rangle$ da lagrangiana, obtemos

$$
\begin{aligned}
-\frac{g^{2}}{4} f^{a b c} f^{a d e}\left\langle A_{\mu}^{b} A_{\nu}^{c} A^{d \mu} A^{e \nu}\right\rangle= & -\frac{g^{2}}{4} f^{a b c} f^{a d e} \frac{\phi_{0}^{4}}{(32)(34)}\left[g_{\mu \nu} g^{\mu \nu} \delta^{b c} \delta^{d e}\right. \\
& \left.+g_{\mu}^{\mu} g_{\nu}^{\nu} \delta^{b d} \delta^{c e}+g_{\mu}^{\nu} g_{\nu}^{\mu} \delta^{b e} \delta^{c d}\right] \\
= & -g^{2} \frac{\phi_{0}^{4}}{(32)(34)}\left[f^{a b b} f^{a d d}+4 f^{a b c} f^{a b c}+f^{a b c} f^{a c b}\right] \\
= & -g^{2} \frac{\phi_{0}^{4}}{(32)(34)}\left[0+4 f^{a b c} f^{a b c}-f^{a b c} f^{a b c}\right],
\end{aligned}
$$

onde usamos a equação (3.2.9) e a propriedade de antissimetria das constantes de estrutura. Usando a equação (3.2.12), obtemos

$$
-\frac{g^{2}}{4} f^{a b c} f^{a d e}\left\langle A_{\mu}^{b} A_{\nu}^{c} A^{d \mu} A^{e \nu}\right\rangle=-\frac{9}{4(34)} g^{2} \phi_{0}^{4} .
$$

Finalmente, substituimos as equações (3.2.13), (3.2.14) e (3.2.11) na equação (3.2.10) e obtemos

$$
\begin{aligned}
\mathcal{L}_{\mathrm{QCD}}= & -\frac{1}{4} \Gamma_{\mu \nu}^{a} \Gamma^{a \mu \nu}-\frac{9}{4(34)} g^{2} \phi_{0}^{4}+\frac{9}{64} g^{2} \mu_{0}^{2} \alpha_{\mu}^{b} \alpha^{b \mu} \\
& +\sum_{q=1}^{N_{f}} \bar{\psi}_{i}^{q}\left\{i \gamma^{\mu}\left[\delta_{i j} \partial_{\mu}-i g T_{i j}^{a} \alpha_{\mu}^{a}\right]-\delta_{i j} m_{q}\right\} \psi_{j}^{q} .
\end{aligned}
$$

\subsubsection{Aproximação para os hard glúons}

Na matéria de quarks (QGP frio) a densidade de quarks é muito alta. Eles são fontes intensas dos campos de glúons. Vamos supor que o número de ocupação dos estados dos hard glúons seja suficientemente grande em todos os níveis de energia e ele possa ser considerado um campo clássico [55, 56]. Matematicamente, essa aproximação consiste em fazer

$$
\alpha_{\mu}^{a} \rightarrow\left\langle\alpha_{\mu}^{a}\right\rangle=\alpha_{0}^{a} \delta_{\mu 0},
$$

sendo $\alpha_{0}^{a}$ constante. Substituindo essa equação na equação (3.2.7),

$$
\begin{aligned}
\Gamma^{a \mu \nu} & =\partial^{\mu} \alpha^{a \nu}-\partial^{\nu} \alpha^{a \nu}+g f^{a b c} \alpha^{b \mu} \alpha^{c \nu} \\
& =g f^{a b c} \alpha_{0}^{b} \alpha_{0}^{c},
\end{aligned}
$$

onde tomamos $\partial^{\mu} \alpha^{a \nu}=0$, pois $\alpha^{a \nu}$ é constante. Substituindo na lagrangiana, obtemos

$$
\begin{aligned}
\mathcal{L}_{\mathrm{QCD}}= & -\frac{g^{2} f^{a b c} f^{a d e}}{4} \alpha_{0}^{b} \alpha_{0}^{c} \alpha_{0}^{d} \alpha_{0}^{e}-\frac{9}{4(34)} g^{2} \phi_{0}^{4}+\frac{9}{64} g^{2} \mu_{0}^{2} \alpha_{0}^{b} \alpha_{0}^{b} \\
& +\sum_{q=1}^{N_{f}} \bar{\psi}_{i}^{q}\left(i \delta_{i j} \gamma^{\mu} \partial_{\mu}+g \gamma^{0} T_{i j}^{a} \alpha_{0}^{a}-\delta_{i j} m_{q}\right) \psi_{j}^{q} .
\end{aligned}
$$


Aqui, vemos que o primeiro termo da lagrangiana contém um termo do tipo $f^{a b c} \alpha_{0}^{b} \alpha_{0}^{c}$, e podemos ver que esse termo se anula, já que temos um termo antissimétrico $\left(f^{a b c}\right)$ vezes um termo simétrico $\left(\alpha_{0}^{b} \alpha_{0}^{c}\right)$. Com isso, os termos que sobram da lagrangiana são

$$
\begin{aligned}
\mathcal{L}_{\mathrm{QCD}}= & -\frac{9}{4(34)} g^{2} \phi_{0}^{4}+\frac{9}{64} g^{2} \mu_{0}^{2} \alpha_{0}^{b} \alpha_{0}^{b} \\
& +\sum_{q=1}^{N_{f}} \bar{\psi}_{i}^{q}\left(i \delta_{i j} \gamma^{\mu} \partial_{\mu}+g \gamma^{0} T_{i j}^{a} \alpha_{0}^{a}-\delta_{i j} m_{q}\right) \psi_{j}^{q} .
\end{aligned}
$$

Na lagrangiana acima, temos um termo proporcional a $\alpha_{0}^{b} \alpha_{0}^{b}$, que é o termo de massa do campo dos hard glúons. Assim, vamos definir a variável $m_{G}$ (massa dos hard glúons) como

$$
m_{G}^{2} \equiv \frac{9}{32} g^{2} \mu_{0}^{2}
$$

Além disso, para facilitar a nossa notação, definiremos também

$$
b \equiv \frac{9}{4(34)} g^{2} .
$$

Com isso, a lagrangiana da MFTQCD toma sua forma final:

$$
\mathcal{L}_{\mathrm{QCD}}=-b \phi_{0}^{4}+\frac{m_{G}^{2}}{2} \alpha_{0}^{a} \alpha_{0}^{a}+\sum_{q=1}^{N_{f}} \bar{\psi}_{i}^{q}\left(i \delta_{i j} \gamma^{\mu} \partial_{\mu}+g \gamma^{0} T_{i j}^{a} \alpha_{0}^{a}-\delta_{i j} m_{q}\right) \psi_{j}^{q},
$$

cujos diagramas de Feynman são apresentados na figura 3.5. O primeiro diagrama é referente à interação entre os quarks e os hard gluons; o segundo diagrama, à geração da massa dinâmica $m_{G}$; e o terceiro diagrama, ao termo $b \phi_{0}^{4}[52]$.
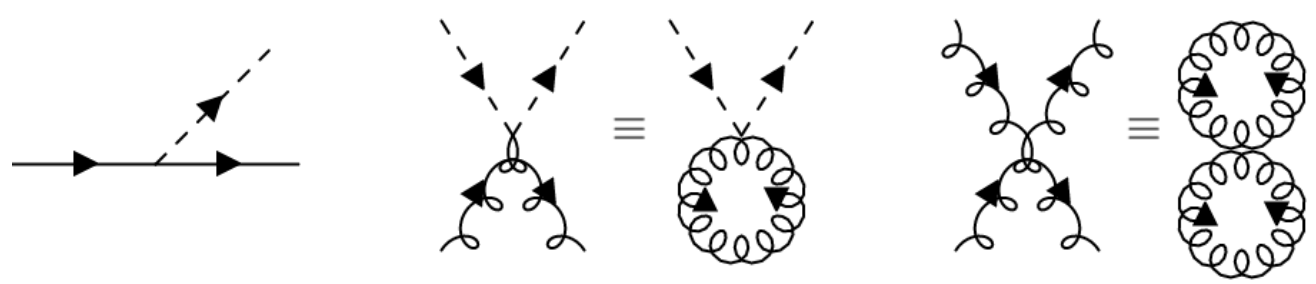

Figura 3.5: Diagramas de Feynamn da equação (3.2.20). Linhas contínuas representam os quarks; linhas tracejadas, os hard glúons; e linhas helicoidais, os soft glúons [52].

\subsubsection{Equação de Estado}

Para obtermos as equações de estado, vamos primeiramente obter as equações de movimento a partir das equações de Euler-Lagrange. 
Para o campo $\bar{\psi}^{q}$, teremos

$$
\frac{\partial \mathcal{L}_{\mathrm{QCD}}}{\partial \bar{\psi}^{q}}-\partial_{\mu} \frac{\partial \mathcal{L}_{\mathrm{QCD}}}{\partial\left(\partial_{\mu} \bar{\psi}^{q}\right)}=0 \Rightarrow\left(i \gamma^{\mu} \partial_{\mu}+g \gamma^{0} T^{a} \alpha_{0}^{a}-m_{q}\right) \psi^{q}=0
$$

Já para o campo $\alpha_{0}^{a}$,

$$
\frac{\partial \mathcal{L}_{\mathrm{QCD}}}{\partial \alpha_{0}^{a}}-\partial_{\mu} \frac{\partial \mathcal{L}_{\mathrm{QCD}}}{\partial\left(\partial_{\mu} \alpha_{0}^{a}\right)}=0 \Rightarrow m_{G}^{2} \alpha_{0}^{a}+g \sum_{q=1}^{N_{f}} \bar{\psi}^{q} \gamma^{0} T^{a} \psi^{q}=0
$$

Relembrando o quadri-vetor densidade de corrente,

$$
j^{a \mu}=\sum_{q=1}^{N_{f}} \bar{\psi}^{q} \gamma^{\mu} T^{a} \psi^{q}
$$

vemos que o último termo da lagrangiana é a componente temporal dessa corrente

$$
\rho^{a} \equiv j^{a 0}=\sum_{q=1}^{N_{f}} \bar{\psi}^{q} \gamma^{0} T^{a} \psi^{q}=\sum_{q=1}^{N_{f}} \psi^{\dagger^{q}} T^{a} \psi^{q},
$$

onde usamos a definição

$$
\bar{\psi} \equiv \psi^{\dagger} \gamma^{0}
$$

e a propriedade $\gamma^{0} \gamma^{0}=1$. Sendo assim, usando a equação (3.2.24) na equação (3.2.22), as equações de movimento do nosso sistema ficam sendo

$$
\begin{aligned}
& \left(i \gamma^{\mu} \partial_{\mu}+g \gamma^{0} T^{a} \alpha_{0}^{a}-m_{q}\right) \psi^{q}=0, \\
& m_{G}^{2} \alpha_{0}^{a}=-g \rho^{a} .
\end{aligned}
$$

Conhecendo as equações de movimento, podemos obter as equações de estado a partir do tensor de energia-momento, sendo que, para o caso de um fluido ideal, ele é dado por

$$
T=\operatorname{diag}\left(\epsilon, P_{X}, P_{Y}, P_{z}\right)
$$

Com isso, podemos escrever a densidade de energia e a pressão do sistema como

$$
\begin{aligned}
\epsilon & =\left\langle T_{00}\right\rangle, \\
P & =\frac{1}{3}\left\langle T_{i i}\right\rangle,
\end{aligned}
$$

onde consideramos que o fluido é isotrópico e $i$ indica as coordenadas espaciais ( $i=$ $1,2,3)$. Para uma lagrangiana que não depende explicitamente das coordenadas, o tensor de energia-momento pode ser obtido através da equação:

$$
T_{\nu}^{\mu}=\sum_{q=1}^{N_{f}} \frac{\partial \mathcal{L}}{\partial\left(\partial_{\mu} \psi^{q}\right)}\left(\partial_{\nu} \psi^{q}\right)-g_{\nu}^{\mu} \mathcal{L}
$$


Com isso e com a equação (3.2.29), podemos escrever a densidade de energia como

$$
\epsilon=\sum_{q=1}^{N_{f}} \frac{\partial \mathcal{L}}{\partial\left(\partial_{0} \psi^{q}\right)}\left(\partial_{0} \psi^{q}\right)-g_{00} \mathcal{L}
$$

Substituindo a equação (3.2.20) nesta última equação, obtemos

$$
\epsilon=\sum_{q=1}^{N_{f}} i \bar{\psi}^{q} \gamma^{0} \partial_{0} \psi^{q}+b \phi_{0}^{4}-\frac{m_{G}^{2}}{2} \alpha_{0}^{a} \alpha_{0}^{a}-\sum_{q=1}^{N_{f}} \bar{\psi}^{q}\left(i \gamma^{\mu} \partial_{\mu}+g \gamma^{0} T^{a} \alpha_{0}^{a}-m_{q}\right) \psi^{q} .
$$

Comparando o último termo com a equação de movimento (3.2.26), vemos que esse se anula. Portanto, a densidade de energia pode ser escrita como

$$
\epsilon=\sum_{q=1}^{N_{f}} i \bar{\psi}^{q} \gamma^{0} \partial_{0} \psi^{q}+b \phi_{0}^{4}-\frac{m_{G}^{2}}{2} \alpha_{0}^{a} \alpha_{0}^{a} .
$$

A fim de reescrever o primeiro termo, vamos multiplicar a equação (3.2.26) por $\bar{\psi}^{q}$ pela esquerda

$$
\begin{aligned}
0 & =\bar{\psi}^{q}\left(i \gamma^{\mu} \partial_{\mu}+g \gamma^{0} T^{a} \alpha_{0}^{a}-m_{q}\right) \psi^{q} \\
& =i \bar{\psi}^{q} \gamma^{\mu} \partial_{\mu} \psi^{q}+g \alpha_{0}^{a} \bar{\psi}^{q} \gamma^{0} T^{a} \psi^{q}-m_{q} \bar{\psi}^{q} \psi^{q} \\
& =i \bar{\psi}^{q} \gamma^{0} \partial_{0} \psi^{q}+i\left(\psi^{q}\right)^{\dagger} \vec{\gamma} \cdot \nabla \psi^{q}+g \alpha_{0}^{a}\left(\left(\psi^{q}\right)^{\dagger} \gamma^{0} \gamma^{0} T^{a} \psi^{q}\right)-m_{q}\left(\psi^{q}\right)^{\dagger} \gamma^{0} \psi^{q}
\end{aligned}
$$

Aplicando a somatória e isolando o termo desejado,

$$
\sum_{q=1}^{N_{f}} i \bar{\psi}^{q} \gamma^{0} \partial_{0} \psi^{q}=\sum_{q=1}^{N_{f}}\left(\psi^{q}\right)^{\dagger}\left(-i \vec{\alpha} \cdot \nabla+\gamma^{0} m_{q}\right) \psi^{q}-g \alpha_{0}^{a} \rho^{a},
$$

onde usamos que $\rho^{a}=\sum_{q=1}^{N_{f}}\left(\psi^{q}\right)^{\dagger} T^{a} \psi^{q}$ (equação (3.2.24)) e

$$
\vec{\alpha}=\gamma^{0} \vec{\gamma}=\left[\begin{array}{ll}
0 & \vec{\sigma} \\
\vec{\sigma} & 0
\end{array}\right]
$$

Substituindo esse termo em $\epsilon$, obtemos

$$
\epsilon=\sum_{q=1}^{N_{f}}\left(\psi^{q}\right)^{\dagger}\left(-i \vec{\alpha} \cdot \nabla+\gamma^{0} m_{q}\right) \psi^{q}-g \alpha_{0}^{a} \rho^{a}+b \phi_{0}^{4}-\frac{m_{G}^{2}}{2} \alpha_{0}^{a} \alpha_{0}^{a} .
$$

Com a densidade de energia escrita desta forma, podemos substituir a equação

$$
\left(\psi^{q}\right)^{\dagger}\left(-i \vec{\alpha} \cdot \nabla+\gamma^{0} m_{q}\right) \psi^{q}=3 \frac{\gamma_{Q}}{2 \pi^{2}} \int_{0}^{k_{F, q}} d k_{q} \vec{k}_{q}^{2} \sqrt{\vec{k}_{q}^{2}+m_{q}^{2}},
$$

deduzida no apêndice D. Com isso, obtemos

$$
\epsilon=-g \alpha_{0}^{a} \rho^{a}+b \phi_{0}^{4}-\frac{m_{G}^{2}}{2} \alpha_{0}^{a} \alpha_{0}^{a}+3 \frac{\gamma_{Q}}{2 \pi^{2}} \sum_{q=1}^{N_{f}} \int_{0}^{k_{F, q}} d k_{q} \vec{k}_{q}^{2} \sqrt{\vec{k}_{q}^{2}+m_{q}^{2}}
$$


onde $k_{F, q}$ é o momento de Fermi que pode ser relacionado com a densidade do número de quarks pela equação

$$
\rho=\frac{\gamma_{Q}}{2 \pi^{2}} \sum_{q=1}^{N_{f}} k_{F, q}^{3}
$$

também deduzida no apêndice D. Podemos eliminar o campo $\alpha_{0}^{a}$ usando a equação (3.2.27). Fazendo isso, obtemos

$$
\epsilon=+\frac{g^{2}}{2 m_{G}^{2}} \rho^{a} \rho^{a}+b \phi_{0}^{4}+3 \frac{\gamma_{Q}}{2 \pi^{2}} \sum_{q=1}^{N_{f}} \int_{0}^{k_{F, q}} d k_{q} \vec{k}_{q}^{2} \sqrt{\vec{k}_{q}^{2}+m_{q}^{2}},
$$

Além disso, podemos relacionar a densidade de cor dos quarks $\left(\rho^{a}\right)$ com a densidade de número de quarks $(\rho)$. Pela equação (3.2.24), temos que

$$
\begin{aligned}
\rho^{a} \rho^{a} & =\sum_{q=1}^{N_{f}} \sum_{q^{\prime}=1}^{N_{f}}\left(\left(\psi_{i}^{q}\right)^{\dagger} T_{i j}^{a} \psi_{j}^{q}\right)\left(\left(\psi_{k}^{q^{\prime}}\right)^{\dagger} T_{k l}^{a} \psi_{l}^{q^{\prime}}\right) \\
& =\sum_{q=1}^{N_{f}} \sum_{q^{\prime}=1}^{N_{f}}\left(c_{i}^{\dagger} T_{i j}^{a} c_{j}\right)\left(\psi^{q}\right)^{\dagger} \psi^{q}\left(c_{k}^{\dagger} T_{k l}^{a} c_{l}\right)\left(\psi^{q^{\prime}}\right)^{\dagger} \psi^{q^{\prime}} \\
& =3 \sum_{q=1}^{N_{f}} \rho^{q} \sum_{q^{\prime}=1}^{N_{f}} \rho^{q^{\prime}} \\
& =27 \rho_{B}^{2},
\end{aligned}
$$

onde usamos que $\left(\psi^{q}\right)^{\dagger} \psi^{q}=\rho^{q}$ e $\psi_{i}^{q}=\psi^{q} c_{i}$, com $c_{i}$ o vetor de cor (ver apêndice D), o resultado do produto $\left(c_{i}^{\dagger} T_{i j}^{a} c_{j}\right)\left(c_{k}^{\dagger} T_{k l}^{a} c_{l}\right)=3 \mathrm{e}$

$$
\rho_{B}=\frac{1}{3} \sum_{q=1}^{N_{f}} \rho^{q}
$$

sendo a densidade bariônica (já que os quarks possuem carga bariônica igual a 1/3). Substituindo na densidade de energia, temos que

$$
\epsilon=+\frac{27 g^{2}}{2 m_{G}^{2}} \rho_{B}^{2}+b \phi_{0}^{4}+3 \frac{\gamma_{Q}}{2 \pi^{2}} \sum_{q=1}^{N_{f}} \int_{0}^{k_{F, q}} d k_{q} \vec{k}_{q}^{2} \sqrt{\vec{k}_{q}^{2}+m_{q}^{2}},
$$

Por fim, podemos calcular a integral, e assim obtemos a expressão final para a densidade de energia

$$
\begin{aligned}
\epsilon= & \frac{27 g^{2}}{2 m_{G}^{2}} \rho_{B}^{2}+b \phi_{0}^{4}+3 \frac{\gamma_{Q}}{2 \pi^{2}} \sum_{q=1}^{N_{f}}\left[\frac{1}{4} k_{F, q}^{3} \sqrt{k_{F, q}^{2}+m_{q}^{2}}\right. \\
& \left.+\frac{1}{8} m_{q}^{2} k_{F, q} \sqrt{k_{F, q}^{2}+m_{q}^{2}}-\frac{m_{q}^{4}}{8} \ln \left(\frac{k_{F, q}+\sqrt{k_{F, q}^{2}+m_{q}^{2}}}{m_{q}}\right)\right] .
\end{aligned}
$$


Para obtermos a pressão, precisamos calcular o termo $T_{i i}$ da equação (3.2.31). Para isso, substituimos a lagrangiana (3.2.20) no termo $T_{i i}$, obtendo assim

$$
\begin{aligned}
T_{i i} & =-\sum_{q=1}^{N_{f}} i \bar{\psi}^{q} \gamma^{i} \partial_{i} \psi^{q}-b \phi_{0}^{4}+\frac{m_{G}^{2}}{2} \alpha_{0}^{a} \alpha_{0}^{2}+\sum_{q=1}^{N_{f}} \bar{\psi}^{q}\left(i \gamma^{\mu} \partial_{\mu}+g \gamma^{a} T^{a} \alpha_{0}^{a}-m_{q}\right) \psi^{q} \\
& =-\sum_{q=1}^{N_{f}} i\left(\psi^{q}\right)^{\dagger} \vec{\alpha} \cdot \nabla \psi^{q}-b \phi_{0}^{4}+\frac{27 g^{2}}{2 m_{G}^{2}} \rho_{B}^{2},
\end{aligned}
$$

onde usamos a equação de movimento (3.2.26) para anular o último termo e a equação de movimento (3.2.27) e a relação (3.2.41) para escrever o campo $\alpha_{0}^{a}$ em termos da densidade bariônica. Podemos reescrever o primeiro termo usando o resultado obtido no apêndice $\mathrm{D}$

$$
\left(\psi^{q}\right)^{\dagger}(-i \vec{\alpha} \cdot \nabla) \psi^{q}=3 \frac{\gamma_{Q}}{2 \pi^{2}} \int_{0}^{k_{F, q}} d k_{q} \frac{\vec{k}_{q}^{4}}{\sqrt{\vec{k}_{q}^{2}+m_{q}^{2}}},
$$

e em seguida, usando a equação (3.2.30), obtemos que a pressão pode ser escrita como

$$
\begin{aligned}
P= & \frac{27 g^{2}}{2 m_{G}^{2}} \rho_{B}^{2}-b \phi_{0}^{4}+\frac{\gamma_{Q}}{2 \pi^{2}} \sum_{q=1}^{N_{f}}\left[\frac{1}{4} k_{F, q}^{3} \sqrt{k_{F, q}^{2}+m_{q}^{2}}\right. \\
& \left.-\frac{3}{8} m_{q}^{2} k_{F, q} \sqrt{k_{F, q}^{2}+m_{q}^{2}}+\frac{3 m_{q}^{4}}{8} \ln \left(\frac{k_{F, q}+\sqrt{k_{F, q}^{2}+m_{q}^{2}}}{m_{q}}\right)\right],
\end{aligned}
$$

onde resolvemos a integral presente na equação (3.2.45).

Analisando os termos das equações de estado, vemos que o primeiro termo vem da interação dos "hard" glúons com os quarks. Esse termo gera mais pressão no nosso sistema, de forma que as equações de estado sejam mais duras (e portanto, permitam um valor de massa máxima maior para as estrelas de nêutrons). Já o segundo termo, vem dos "soft" glúons. Este é um termo constante, que adquire valores positivos na densidade de energia, e negativos na pressão. Comparando com o modelo de sacola do MIT, podemos concluir que o termo dos "soft" glúons tem o mesmo comportamento da constante de sacola do MIT. Assim, para preservar a analogia, vamos introduzir a seguinte notação:

$$
B=b \phi_{0}^{4}
$$

Os últimos termos da densidade de energia e pressão são os de um gás de Fermi dos quarks. Para usar a MFTQCD no estudo da estrutura estelar, consideramos também uma contribuição vinda dos elétrons, que trataremos como um gás de Fermi. Com isso, rees- 
crevendo as equações de estados, temos que

$$
\begin{aligned}
P= & \frac{27 g^{2}}{2 m_{G}^{2}} \rho_{B}^{2}-B \\
& +\frac{\gamma_{Q}}{8 \pi^{2}} \sum_{q=u, d, s}\left[k_{F, q} \mu_{F, q}\left(\mu_{F, q}^{2}-\frac{5}{2} m_{q}^{2}\right)+\frac{3}{2} m_{q}^{4} \ln \left(\frac{k_{F, q}+\mu_{F, q}}{m_{q}}\right)\right] \\
& +\frac{\gamma_{e}}{24 \pi^{2}}\left[k_{F, e} \mu_{F, e}\left(\mu_{F, e}^{2}-\frac{5}{2} m_{e}^{2}\right)+\frac{3}{2} m_{e}^{4} \ln \left(\frac{k_{F, e}+\mu_{F, e}}{m_{e}}\right)\right] \\
\epsilon= & \frac{27 g^{2}}{2 m_{G}^{2}} \rho_{B}^{2}+B \\
& +3 \frac{\gamma_{Q}}{8 \pi^{2}} \sum_{q=u, d, s}\left[k_{F, q} \mu_{F, q}\left(\mu_{F, q}^{2}-\frac{1}{2} m_{q}^{2}\right)-\frac{1}{2} m_{q}^{4} \ln \left(\frac{k_{F, q}+\mu_{F, q}}{m_{q}}\right)\right] \\
& +\frac{\gamma_{e}}{8 \pi^{2}}\left[k_{F, e} \mu_{F, e}\left(\mu_{F, e}^{2}-\frac{1}{2} m_{e}^{2}\right)-\frac{1}{2} m_{e}^{4} \ln \left(\frac{k_{F, e}+\mu_{F, e}}{m_{e}}\right)\right] \\
\rho_{B}= & \frac{\gamma_{Q}}{6 \pi^{2}} \sum_{q=u, d, s} k_{F, q}^{3},
\end{aligned}
$$

onde $\mu_{F, i}=\sqrt{k_{F, i}^{2}+m_{i}}, \gamma_{e}=2$ e $\gamma_{Q}=2$, este último devido ao fato da soma nas cores já ter sido feita. Nem a constante de acoplamento g, nem a massa do gluon, $m_{G}$, aparecem separadas. Assim, a partir de agora, vamos usar a variável $\xi \equiv g / m_{G}$.

\subsubsection{Resultados}

Para usar as equações de estado no estudo da estrutura estelar, precisamos antes determinar a janela de valores dos parâmetros livres; ou seja, precisamos verificar para quais conjuntos de valores das variáveis $\xi$ e $B$ as condições de estabilidade (equações (3.0.1) e $(3.0 .2))$

$$
\left.\frac{\epsilon}{\rho_{B}}\right|_{u, d, s} \leq 934 \mathrm{MeV} \leq\left.\frac{\epsilon}{\rho_{B}}\right|_{u, d},
$$

são satisfeitas pelo menos quando a pressão for nula. Para isso, considerando os quarks up, down e strange (matéria estranha) e fixando um valor de $B$, é possível obter o valor de $\xi$ que satisfaça a condição de $P=0$ e o valor da condição limite da estabilidade

$$
\left.\frac{\epsilon}{\rho_{B}}\right|_{u, d, s}=934 \mathrm{MeV} .
$$

Repetindo esse processo para diferentes valores de $B$, obtemos a linha do limite da estabilidade para a matéria estranha, apresentada pela linha sólida na figura 3.6. Dessa forma, os conjutos de valores $(B, \xi)$ que se situam acima dessa linha obtida, resultam em valores tais que $\epsilon /\left.\rho_{B}\right|_{u, d, s}>934 \mathrm{MeV}$, e portanto, a condição de estabilidade não é satisfeita. Fazendo o mesmo procedimento, porém considerando apenas os quarks up e down (matéria 


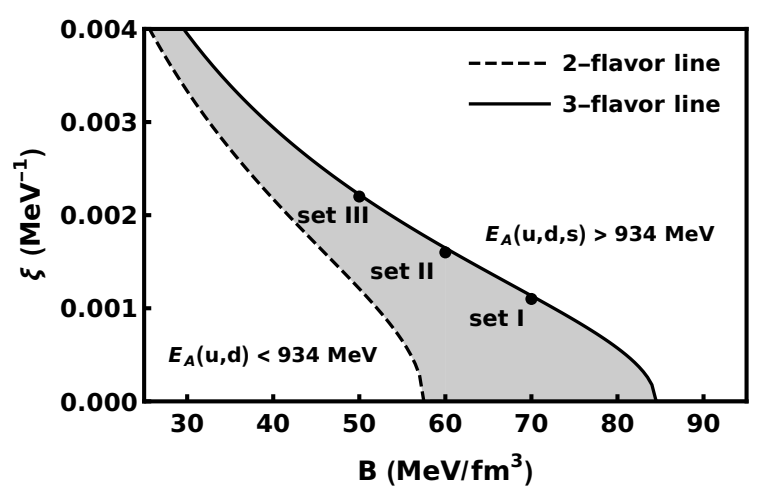

Figura 3.6: Gráfico da estabilidade da MFTQCD.

de quarks), obtemos a linha tracejada da figura 3.6. Nesse caso, os conjuntos de valores $(B, \xi)$ que se situam abaixo dessa linha, resultam em valores tais que $\epsilon /\left.\rho_{B}\right|_{u, d}<934$ $\mathrm{MeV}$, e portanto, a condição de estabilidade não é satisfeita. Juntando as duas condições de estabilidade, concluímos que qualquer conjunto de valor de $\xi$ e $B$ escolhido dentro da área rachurada da figura 3.6, satisfaz, simultaneamente, as condições de estabilidades dadas pelas equações (3.0.1) e (3.0.2). Tendo definida a janela de estabilidade dos parâmetros livres $\xi$ e $B$, escolhemos três conjuntos de valores possíveis, dados pela tabela 3.2.

\begin{tabular}{|ccc|}
\hline Set & $\mathrm{B}\left(\mathrm{MeV} / \mathrm{fm}^{3}\right)$ & $\xi\left(\mathrm{MeV}^{-1}\right)$ \\
\hline \hline I & 70 & 0.0011 \\
\hline II & 60 & 0.0016 \\
\hline III & 50 & 0.0022 \\
\hline
\end{tabular}

Tabela 3.2: Janela de estabilidade dos parâmetros livres da MFTQCD.

Uma vez que fixamos os valores dos parâmetros livres, podemos fazer um gráfico com as equações de estado. Para comparar a MFTQCD com outros modelos, usaremos uma versão melhorada do modelo de sacola do MIT apresentada em [57, 19, 20], que é dada pela equação

$$
P(\epsilon)=\frac{\left(\epsilon-B_{\mathrm{eff}}\right)}{3}-\frac{a_{2}^{2}}{12 \pi^{2} a_{4}}\left[1+\sqrt{1+\frac{16 \pi^{2} a_{4}}{a_{2}^{2}}\left(\epsilon-B_{\mathrm{eff}}\right)}\right],
$$

onde escolhemos os valores de $B_{\text {eff }}^{1 / 4}=142.52 \mathrm{MeV}, a_{2}^{1 / 2}=100 \mathrm{MeV}, a_{4}=0.535$. Neste modelo, além da constante de sacola $B_{\text {eff }}$ representando a energia do vácuo, temos também as constantes $a_{2}$ e $a_{4}$. Esta primeira está relacionada com o fenômeno da supercondutividade de cor e a segunda, com as interações fortes na pressão do mar de Fermi livre de quarks $[57,19,20]$. Sendo assim, podemos visualizar as equações de estado no gráfico da figura 3.7 . 


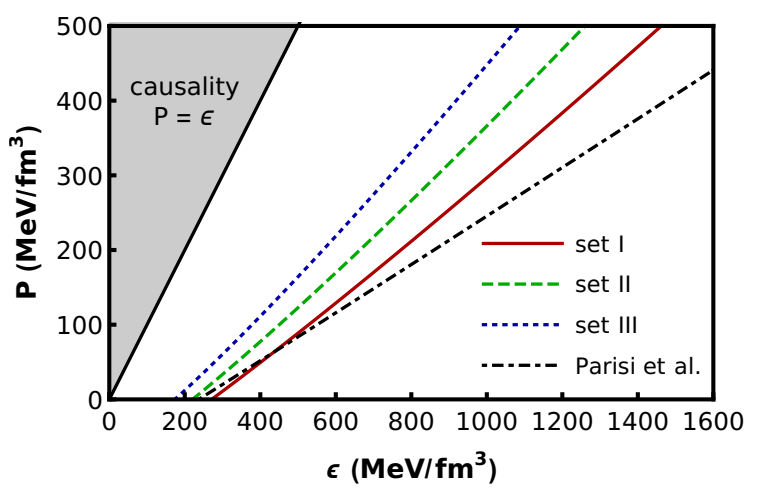

Figura 3.7: Pressão em função da densidade de energia. As linhas coloridas são os gráficos das equações de MFTQCD e a linha traço-ponto em preto, é o modelo de comparação apresentado em [20].

Nesse gráfico, vemos que as equações da MFTQCD se apresentam com mais pressão do que o modelo genérico do MIT, porém sem violar a causalidade. A principal diferença entre a MFTQCD e o modelo de sacola do MIT está no primeiro termo das equações (3.2.48) e (3.2.49), que é proporcional a $\rho_{B}^{2}$. Como pode ser visto em (3.2.40), este termo vem da interação quártica efetiva (interação entre as duas corrente $\rho^{a} \rho^{a}$ ) que surge quando o campo dos hard gluons é eliminado com o uso da equação de movimento. O sinal desta interação é tal que ela é repulsiva e assim contribui para o aumento da pressão.

Sabendo a relação entre pressão e densidade de energia, podemos resolver as equações de TOV e obter a relação massa-raio e a deformabilidade de maré, apresentadas na figura 3.8 .
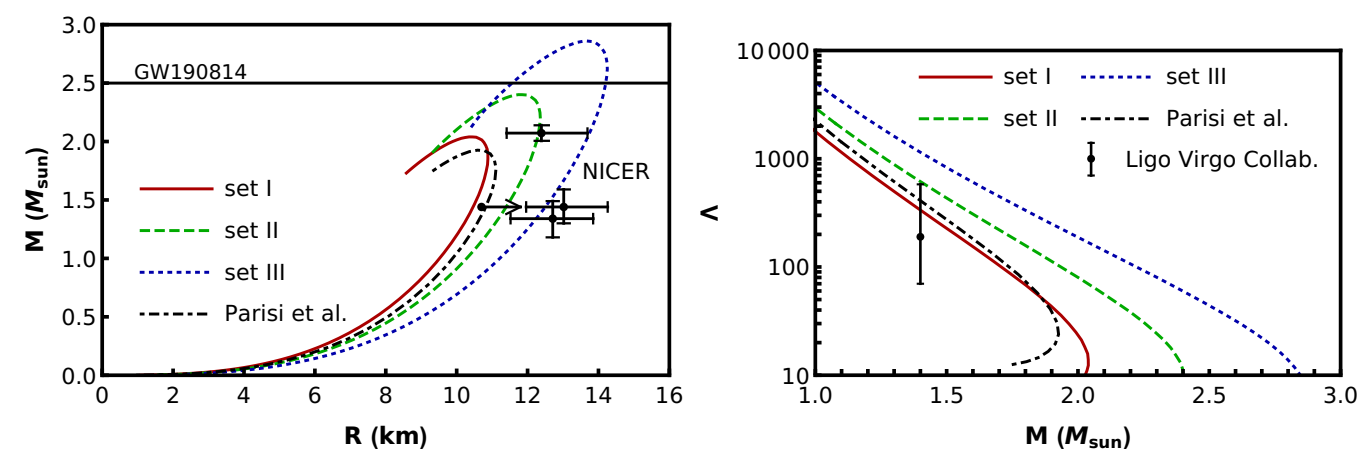

Figura 3.8: Relação massa-raio à esquerda e deformabilidade de maré à direita para uma estrela de quarks cujos constituintes são descritos pela MFTQCD.

No diagrama massa-raio, vemos que todas as equações da MFTQCD descrevem bem os dados experimentais conhecidos, apesar de apenas o set III alcançar o valor de $2.5 \mathrm{M}_{\odot}$ (porém, relembramos que não há um consenso se o objeto compacto observado no evento GW190814 é, de fato, uma estrela de nêutrons ou um buraco negro). No gráfico da defor- 
mabilidade de maré, vemos que os conjunto I e II estão de acordo com o dado experimental. Não excluímos o conjunto III porque o ponto experimental tem $90 \%$ de credibilidade. Assim, o conjunto III não é incompatível com os dados, apenas menos provável. É interessante observar que existe uma tensão entre os dados do diagrama massa-raio e da deformabilidade de maré. Os modelos que melhor descrevem os pontos experimentais do diagrama massa-raio tendem a superestimar a deformabilidade de maré. 


\section{Estrelas de Hádrons}

Inicialmente, as estrelas de nêutrons foram propostas por Oppenheimer e Volkoff como objetos feitos de um gás ideal de nêutrons [8]. Entretanto, sabemos que os nêutrons são instáveis e decaem em prótons, elétrons e neutrinos atráves do decaimento beta. $\mathrm{O}$ primeiro modelo que vamos estudar neste capítulo é o modelo do gás de Fermi. Nele, as estrelas de nêutrons são formadas por um gás ideal de prótons, nêutrons e elétrons (seção 4.1). Veremos que este modelo não gera pressão suficiente, resultando em estrelas com massas muito pequenas. Além disso, veremos também que o modelo de gás de Fermi não satisfaz o limite de baixas energias, obtido através de cálculos com teoria efetiva quiral. A seguir vamos estudar um modelo que, além de satisfazer o limite da teorial efetiva quiral, gera maior pressão e resulta em estrelas com massas maiores (seção 4.2). Ele foi retirado da referência [3] e será chamado de modelo HLPS.

\subsection{Modelo de Gás de Fermi}

Neste primeiro modelo, levamos em consideração as seguintes hipóteses [8]:

1. O gás que forma as estrelas de nêutrons é um gás ideal (sem interações) constituído por nêutrons, prótrons e elétrons.

2. A temperatura do gás é nula; ou, equivalentemente, dizemos que o gás é degenerado: todos os estados quânticos estão ocupados até um dado valor de energia $E_{F}$.

3. As proporções de nêutrons, prótons e elétrons são tais que satisfazem o que chamamos de equilíbrio beta: não ocorre o decaimento beta e nem a sua reação inversa.

4. As proporções das partículas devem satisfazer também a neutralidade elétrica. 
O equilíbrio beta se justifica pelo fato de que as componentes do gás respeitam o princípio de Pauli. Conforme os nêutrons vão decaindo, os estados menos energéticos dos prótons e elétrons vão sendo ocupados. E conforme esses níveis de energia vão sendo preenchidos, os nêutrons menos energéticos não vão conseguir decair - já que essa quantidade de energia baixa permite o nêutron decair em prótons e elétrons cujos níveis já estão ocupados. Assim, os nêutrons precisam ser cada vez mais energéticos para conseguir decair. $\mathrm{O}$ mesmo acontecerá com a reação inversa. O equilíbrio beta será atingido quando as proporções de nêutrons, prótons e elétrons serão tais que não existam nêutrons com energia o suficiente para decair, nem prótons e elétrons com energia para ocorrer a reação inversa.

Analogamente ao que foi feito na seção 3.1 para o gás de quarks, no limite em que a temperatura é nula, as equações de estado para os hádrons são dadas por [8, 58, 59]

$$
\begin{aligned}
P_{H} & =\frac{1}{12 \pi^{2}} \sum_{i=p, n, e}\left[\mu_{F, i} k_{F, i}\left(\mu_{F, i}^{2}-\frac{5}{2} m_{i}^{2}\right)+\frac{3}{2} m_{i}^{4} \ln \left(\frac{\mu_{F, i}+k_{F, i}}{m_{i}}\right)\right] ; \\
\epsilon_{H} & =\frac{1}{4 \pi^{2}} \sum_{i=p, n, e}\left[\mu_{F, i} k_{F, i}\left(\mu_{F, i}^{2}-\frac{1}{2} m_{i}^{2}\right)-\frac{1}{2} m_{i}^{4} \ln \left(\frac{\mu_{F, i}+k_{F, i}}{m_{i}}\right)\right] ; \\
\rho_{B} & =\frac{1}{3 \pi^{2}} \sum_{q=p, n} k_{F, i}^{3},
\end{aligned}
$$

onde

$$
\mu_{F, i}=\sqrt{k_{F, i}^{2}+m_{i}^{2}}
$$

são os potenciais químicos de Fermi e $k_{F, i}$, os momentos de Fermi. Assim como explicado também na seção 3.1, precisamos relacionar os momentos de Fermi e a densidade bariônica para sermos capazes de resolver essas equações de estado. Sendo assim, impomos a condição de neutralidade elétrica

$$
\rho_{p}=\rho_{n} \Rightarrow \frac{1}{3 \pi^{2}} k_{F, p}^{3}=\frac{1}{3 \pi^{2}} k_{F, e}^{3} .
$$

Além disso, consideramos também o decaimento beta, dado por

$$
n \rightarrow p+e+\nu_{e}
$$

que, usando a equação (3.1.11), obtemos

$$
\mu_{F, n}=\mu_{F, p}+\mu_{F, e}
$$

Resolvendo numericamente o sistema de equações formado pelas equações (4.1.3), (4.1.4) e (4.1.5)

$$
\left\{\begin{array}{l}
\rho_{B}=\frac{1}{3 \pi^{2}}\left(k_{F, p}^{3}+k_{F, n}^{3}\right), \\
k_{F, p}=k_{F, e} \\
\sqrt{k_{F, n}^{2}+m_{n}^{2}}=\sqrt{k_{F, p}^{2}+m_{p}^{2}}+\sqrt{k_{F, e}^{2}+m_{e}^{2}},
\end{array}\right.
$$


conseguimos obter os valores dos momentos de Fermi para um dado valor de densidade bariônica. Com estes valores, obtemos os seus potenciais químicos de Fermi respectivos e substituímos os valores encontrados nas equações (4.1.1) e (4.1.2) para encontrar o valor da pressão e densidade de energia. Repentindo esse procedimento para diferentes valores de $\rho_{B}$, construímos os gráficos apresentados nas figuras 4.1 e 4.2. Com a equação de
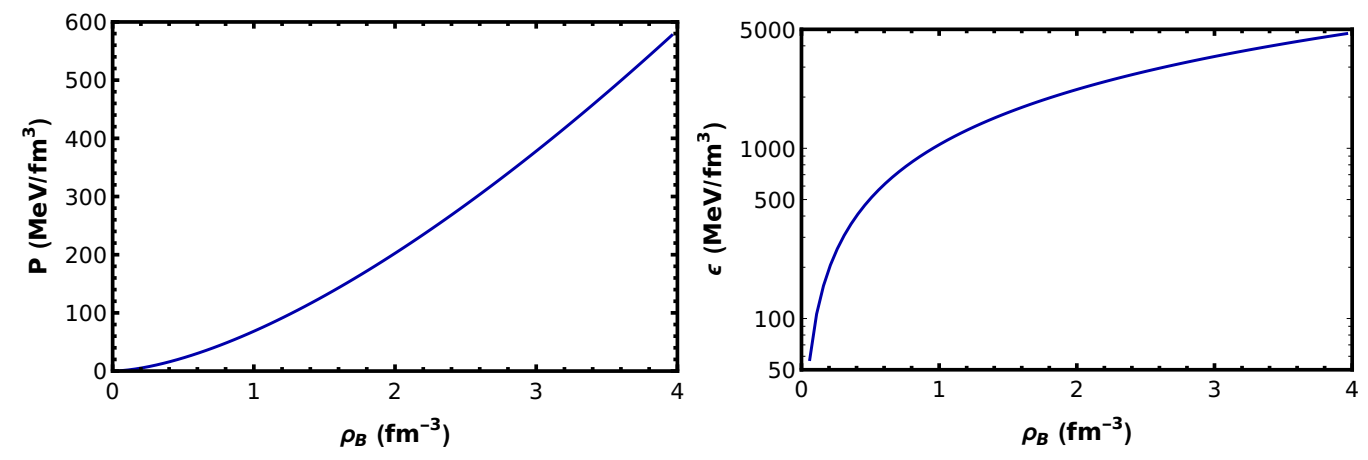

Figura 4.1: Pressão (à esquerda) e densidade de energia (à direita) em função da densidade bariônica do modelo do gás de Fermi.

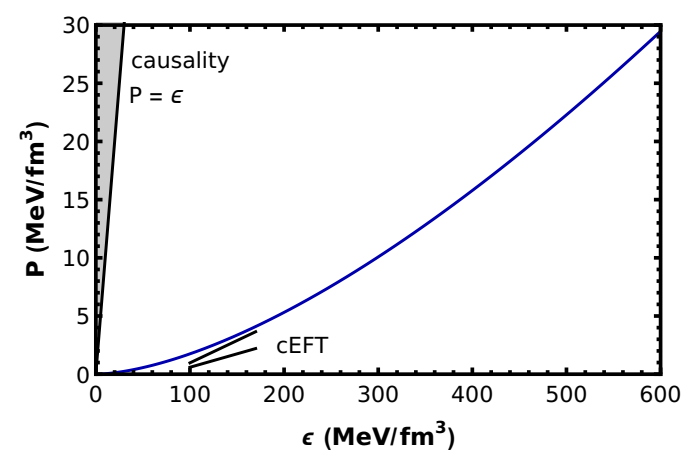

Figura 4.2: Pressão em função da densidade de energia do modelo do gás de Fermi. Em preto, temos o resultado obtido pela teoria efetiva quiral [3].

estado do modelo de gás de Fermi, resolvemos as equações de TOV juntamente com as quantidades necessárias para obter a deformabilidade de maré da estrela. Os resultados estão na figura 4.3.

Nos gráficos apresentados na figura 4.3, vemos que o modelo de gás de Fermi não reproduz nem os dados experimentais de massa-raio e nem da deformabilidade de maré. Isto acontece porque o gás de Fermi gera pouca pressão no sistema, fazendo com que as estrelas de nêutrons compostas por esse gás não suportem massas acima de $0.7 \mathrm{M}_{\odot}$. Além disso, vemos já no gráfico da equação de estado (figura 4.1), que o modelo não satisfaz o limite de baixa densidade de energia, obtido através da teoria efetiva quiral. Verificamos assim que o modelo do gás de Fermi não descreve de forma satisfatória as propriedades conhecidas das estrelas de nêutrons. 

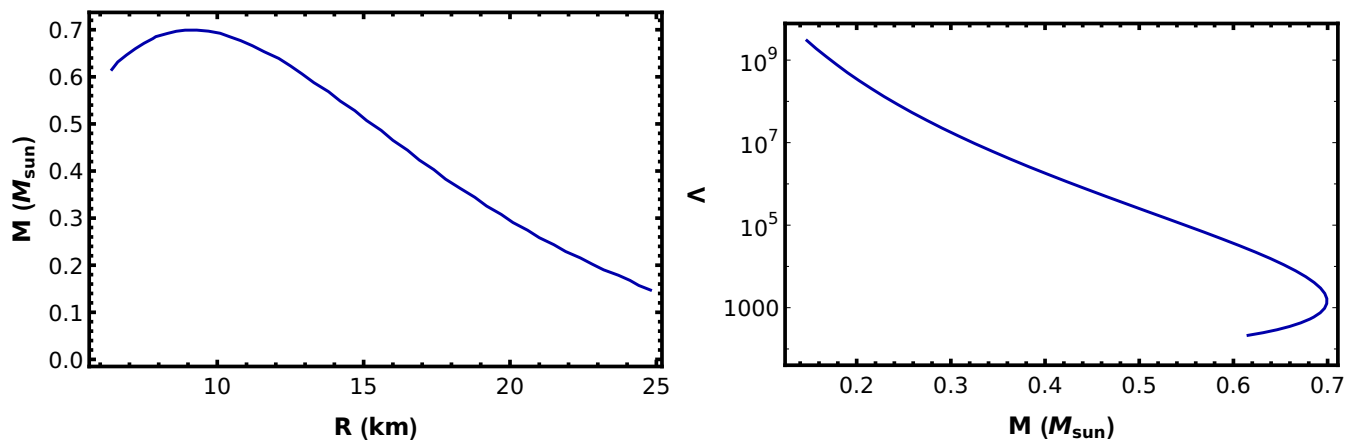

Figura 4.3: Relação massa-raio à esquerda e deformabilidade de maré à direita para uma estrela de nêutrons cujos constituintes são descritos pelo modelo de gás de Fermi. O diagrama massa-raio obtido está compatível com o resultado de Oppenheimer e Volkoff em 1939 [9].

\subsection{Modelo de HLPS}

Uma equação de estado hadrônica que satisfaz o limite de baixa densidade foi proposta nas referências $[3,16]$. Neste artigo, os autores fazem cálculos com teoria de campo efetiva quiral (cEFT, do inglês: chiral effective field theory) e incorporam o equilíbrio beta. Como resultado, encontram a banda azul mostrada no gráfico da figura 4.4. Em [3,16], até o valor de $\rho_{\text {crust }}$, é utilizada a equação denominada de crust EOS (BPS), retirada de [60]. Entre os valores de $\rho_{\text {crust }}$ e $\rho_{1}$, é utilizado o limite superior ou inferior da banda azul (cEFT). A partir de $\rho_{1}$, os cálculos do cEFT não são válidos. Para $\rho>\rho_{1}$, são usadas três equações politrópicas $P=K_{i} \rho^{\Gamma_{i}}$, como mostrado na figura 4.4. As equações de estado

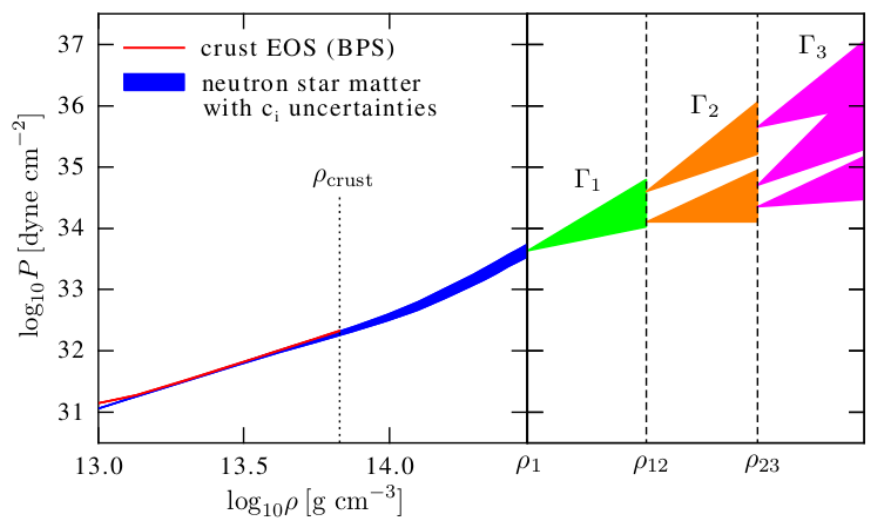

Figura 4.4: Pressão em função da densidade de massa do modelo HLPS. Para valores menores de $\rho_{1}$, temos uma comparação da equação de estado retirada de [60] (em vermelho) com a banda obtida pela cEFT (em azul). Para valores maiores que $\rho_{1}$, temos as três equações politrópicas. Imagem retirada de [3].

utilizadas aqui apresentam algumas diferenças em relação à equação de estado [3, 16]. A 
primeira diferença é que, ao invés de ser utilizada a equação crust EOS (BPS) [60] para densidades baixas, é utilizada a equação SLy(4) [61]. A segunda diferença é referente ao método aplicado para conectar as três equações politrópicas. Em [3, 16], foi utilizado o formalismo denominado por piecewise polytropic (PP) [62]. Apesar deste formalismo produzir bons resultados, ele apresenta alguns problemas. Assim, neste trabalho usamos o formalismo generalized piecewise polytrope (GPP) [63], que é uma aprimoração do formalismo anterior. Diferentemente do formalismo PP, consideramos que, além da pressão, da densidade bariônica e da densidade de energia serem contínuas, a pressão e a densidade de energia devem ser também diferenciáveis nos pontos de intersecção entre as equações de estado (pontos $\rho_{1}, \rho_{12}$ e $\rho_{23}$ ). Isso implica que a velocidade do som $\left(c_{s}^{2}=d P / d \epsilon\right.$ ) se torne uma função contínua. Dessa forma, as equações de estado geradas pelo método GPP acabam sendo mais suaves, mais fáceis de computar numericamente e geram menos erros - principalmente para a deformabilidade de maré, que depende diretamente da velocidade do som. Para que seja possível adicionar essa condição de contorno, as equações politrópicas apresentam um termo a mais, sendo escritas como $P=K_{i} \rho^{\Gamma_{i}}+\Lambda_{i}$ [63]. Os valores de $\Lambda_{i}$ são obtidos a partir das condições de contorno. As equações de estado obtidas e utilizadas aqui são mostradas nas figuras 4.5 e 4.6. Os valores dos parâmetros utilizados foram $\rho_{1}=10^{13.902} \mathrm{~g} / \mathrm{cm}^{3}, \rho_{12}=10^{14.45} \mathrm{~g} / \mathrm{cm}^{3}, \rho_{23}=10^{14.58} \mathrm{~g} / \mathrm{cm}^{3}$, $K_{1}=10^{-27.22}$ em cgs para as três equações de estado. Os valores dos parâmetros $\Gamma_{i}$ são dados na tabela 4.1. As demais variáveis são obtidas através das condições de contorno, como explicado em [63]. Estes parâmetros foram obtidas de forma que a condição

\begin{tabular}{|c|ccc|}
\hline & $\Gamma_{1}$ & $\Gamma_{2}$ & $\Gamma_{3}$ \\
\hline \hline Soft & 2.752 & 4.5 & 3.5 \\
\hline Intermediate & 2.758 & 6.5 & 3.2 \\
\hline Stiff & 2.764 & 8.5 & 3.2 \\
\hline
\end{tabular}

Tabela 4.1: Valores dos parâmetros das equações de estado do modelo HLPS modificado.

de $M_{\text {máx }} \geq 2 M_{\odot}$ seja respeitada e exigindo que a equação de estado Soft passe pelo limite inferior da cEFT; a equação Intermediate passe pelo centro da região determinada pela cEFT; e a Stiff, passe pelo limite superior da cEFT. Em [3], os autores obtiveram o diagrama massa-raio impondo apenas a condição $M_{\text {máx }} \geq 2 M_{\odot}$. Neste trabalho, resolvemos as equações de TOV a partir das equações de estado e obtemos, além do diagrama massa-raio, o gráfico de deformabilidade de maré. Eles são apresentados na figura 4.7. No diagrama massa-raio podemos notar que, além da condição $M_{\text {máx }} \geq 2 M_{\odot}$ ser satisfeita, as três curvas apresentam um comportamento compatível com os dados experimentais atuais. Já no gráfico da deformabilidade de maré, apenas a curva soft está dentro da incerteza do dado experimental. Entretanto, as curvas intermediate e stiff não são completamente 

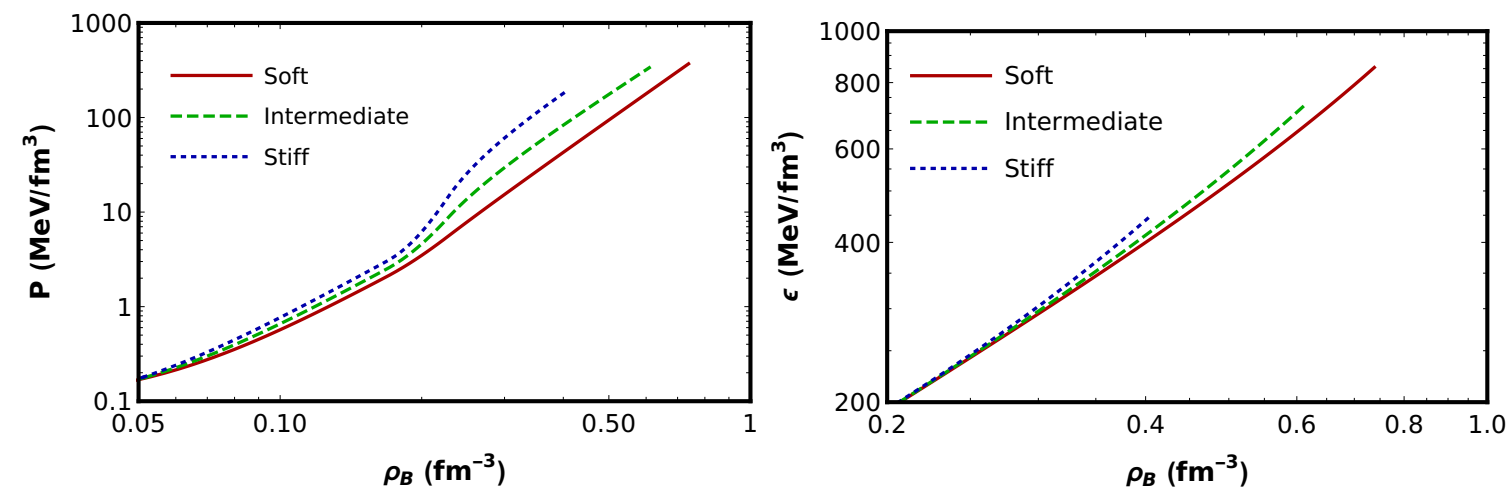

Figura 4.5: Pressão (à esquerda) e densidade de energia (à direita) em função da densidade bariônica do modelo de HLPS.

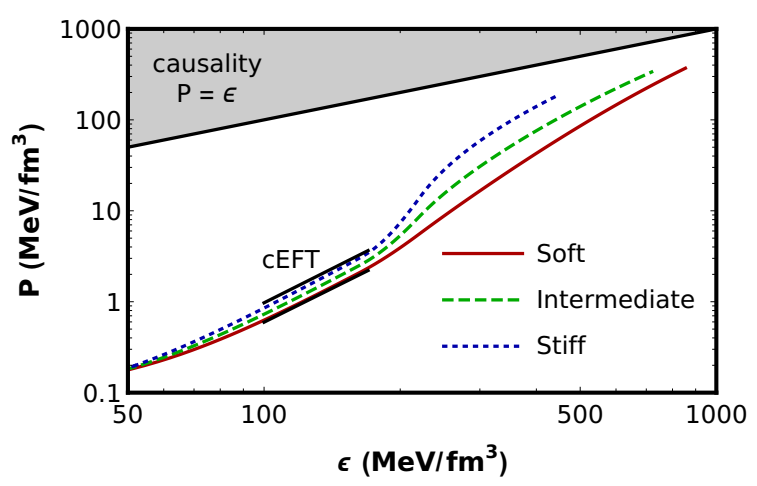

Figura 4.6: Pressão em função da densidade de energia do modelo de HLPS. Em preto, temos o limite de baixa densidade obtido através da teoria efetiva quiral (cEFT) [3].

excluídas, pois o dado experimental tem um nível de credibilidade de 90\%. Com isso, concluímos que as equações de estado desta seção descrevem a matéria densa e fria de forma satisfatória.
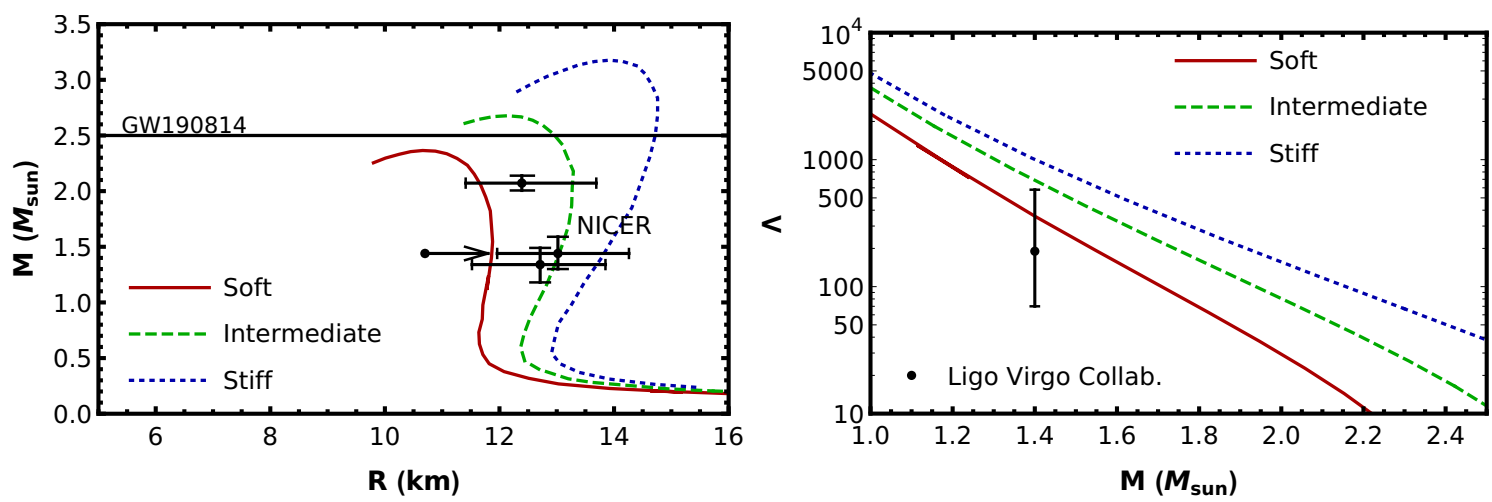

Figura 4.7: Relação massa-raio à esquerda e deformabilidade de maré à direita para uma estrela de nêutrons cujos constituintes são descritos pelo modelo do HLPS. 


\section{Estrelas Híbridas}

Nos dois capítulos anteriores, discutimos estrelas de nêutrons formadas inteiramente por quarks (capítulo 3) e estrelas de nêutrons formadas inteiramente por hádrons (capítulo 4). Entretanto, existe uma terceira possibilidade, que chamamos de estrelas híbridas [8]. Neste caso, a estrela de nêutrons é formada por matéria de quarks na região mais próxima do centro (e portanto, maior densidade bariônica) e por matéria de hádrons na região mais longe do centro (menor densidade bariônica). Em regiões intermediárias, pode haver uma fase mista, onde as duas fases coexistam [8]. A existência dessa região e o seu tamanho depende da forma como ocorre a transição de fase da matéria. Se a transição for mais abrupta (suave), a região de fase mista será menor (maior). Neste contexto, uma questão interessante é: como a natureza desta transição de fase afeta as quantidades observáveis das estrelas de nêutrons? Para responder esta questão, neste capítulo vamos utilizar uma equação composta que nos permite escolher a intensidade da transição de fase. Assim, fixando uma equação de estado para a fase de quarks e uma para a fase de hádrons, construiremos estrelas de nêutrons com diferentes intensidades de transição de fase.

\subsection{Intensidade da Transição de Fase}

A forma mais usual de descrever a fase mista da matéria é através do método de construção de Gibbs ou de Maxwell [64]. Entretanto, podemos encontrar maneiras de simular uma transição de fase, como, por exemplo, através de métodos de interpolação [18], ou de métodos que nos permitem escolher a intensidade da transição de fase [37, 17]. Como um dos objetivos deste trabalho é determinar que mudanças na estrutura estelar ocorrem quando alteramos a forma da transição de fase, vamos usar a equação proposta em [37]. Originalmente, esta equação foi criada para descrever a transição de fase em colisões 
nucleares de alta energia e é dada por [37, 38, 50, 65]

$$
\left(P-P_{Q}\right)\left(P-P_{H}\right)=\delta\left(\rho_{B}\right), \quad \text { sendo } \quad \delta\left(\rho_{B}\right)=\delta_{0} \exp \left[-\left(\rho_{B} / \rho_{c}\right)^{2}\right]
$$

onde $P_{Q}$ e $P_{H}$ são a pressão na fase de quarks e na fase de hadrons, respectivamente ${ }^{1}$. Os parâmetros $\delta_{0}$ e $\rho_{c}$ são parâmetros livres, que determinam a forma da transição de fase, como veremos mais adiante. De modo geral, fixamos o valor de $\delta_{0}$ e variamos $\rho_{c}$ no intervalo:

$$
0 \mathrm{fm}^{-3}<\rho_{c} \leq 1 \mathrm{fm}^{-3}
$$

sendo $\rho_{c} \rightarrow 0$ a transição mais abrupta, em que não há uma região onde haja a coexistências de ambas as fases, e $\rho_{c}=1$, a transição mais suave.

Isolando a pressão da equação (5.1.1), obtemos

$$
P=\frac{1}{2}\left(P_{H}+P_{Q}\right)+\frac{1}{2} \sqrt{\left(P_{Q}-P_{H}\right)^{2}+4 \delta\left(\rho_{B}\right)},
$$

que pode ainda ser reescrita como

$$
\begin{gathered}
P=\lambda P_{H}+(1-\lambda) P_{Q}+\frac{2 \delta\left(\rho_{B}\right)}{\sqrt{\left(P_{Q}-P_{H}\right)^{2}+4 \delta\left(\rho_{B}\right)}}, \\
\epsilon=\lambda \epsilon_{H}+(1-\lambda) \epsilon_{Q}-\frac{2\left[1+\left(\rho_{B} / \rho_{c}\right)^{2}\right] \delta\left(\rho_{B}\right)}{\sqrt{\left(P_{Q}-P_{H}\right)^{2}+4 \delta\left(\rho_{B}\right)}},
\end{gathered}
$$

onde

$$
\lambda \equiv \frac{1}{2}\left[1-\frac{\left(P_{Q}-P_{H}\right)}{\sqrt{\left(P_{Q}-P_{H}\right)^{2}+4 \delta\left(\rho_{B}\right)}}\right],
$$

A densidade de energia pode ser obtida a partir da pressão, através da relação termodinâmica [50]

$$
\epsilon=-\left.\frac{\partial}{\partial \beta} \beta P(T, \mu)\right|_{e^{\beta \mu}},
$$

onde $\beta=1 / T$.

Para estudar a natureza da transição de fase da matéria a partir da equação (5.1.1), vamos, primeiramente, construir estrelas de nêutrons usando o modelo de sacola do MIT com $B=150 \mathrm{MeV} / \mathrm{fm}^{3}$ (seção 3.1) para a fase de quarks, e o modelo do gás de Fermi (seção 4.1) para a fase de hádrons. Como estes modelos geram pouca pressão, não esperamos que os resultados reproduzam as quantidades experimentais. Este primeiro modelo servirá como um estudo dos parâmetros $\rho_{c}$ e $\delta_{0}$ da equação de estado composta. Em seguida, repetiremos o mesmo procedimento usando a equação de estado da MFTQCD (seção 3.2) para a fase de quarks, e a EoS do modelo HLPS (seção 4.2) para a fase de

\footnotetext{
${ }^{1}$ Nas referências [37, 38, 50, 65], a função $\delta$ é definida como $\delta\left(\mu_{B}\right)=\delta_{0} \exp \left[-\left(\mu_{B} / \mu_{c}\right)^{2}\right]$. Entretanto, como este é um estudo preliminar, usamos a variável $\rho_{B}$ no lugar de $\mu_{B}$ para se tornar mais fácil obter uma intuição dos resultados. Em um trabalho futuro, iremos utilizar a equação original com $\delta=\delta\left(\mu_{B}\right)$.
} 
hádrons. Com essas equações, esperamos obter uma estrela híbrida compatível com as observações experimentais mais recentes. Além disso, variando o parâmetro $\rho_{c}$, vamos tentar determinar os efeitos da intensidade de transição de fase na estrutura estelar das estrelas de nêutrons.

\subsubsection{Modelo MIT + gás de Fermi}

Nesta seção, vamos estudar uma estrela híbrida descrita pelas equações de estado (5.1.3) e (5.1.4). A EoS da fase de quarks é dada pelo modelo de sacola do MIT (onde escolhemos $B=150 \mathrm{MeV} / \mathrm{fm}^{3}$ ) e a EoS da fase de hádrons pelo modelo do gás de Fermi. Matematicamente, substituimos as equações de pressão e densidade de energia do modelo do MIT (equações (3.1.6) e (3.1.7)) e do modelo de gás de Fermi (equações (4.1.1) e (4.1.2)) nas funções $P_{Q}, \epsilon_{Q}, P_{H}$ e $\epsilon_{H}$, respectivamente. Em seguida, precisamos escolher os valores dos parâmetros livres $\delta_{0}$ e $\rho_{c}$. É necessário entender que efeitos esses parâmetros causam na equação de estado. Vamos, primeiro, fixar o valor de $\delta_{0}=100\left(\mathrm{MeV} / \mathrm{fm}^{3}\right)^{2}$ e usar os valores de $\rho_{c}=0.2,0.4,1.0 \mathrm{fm}^{-3}$. Com estas escolhas, encontramos as equações de estado apresentadas na figura 5.1. Na figura vemos que na região de baixa densidade

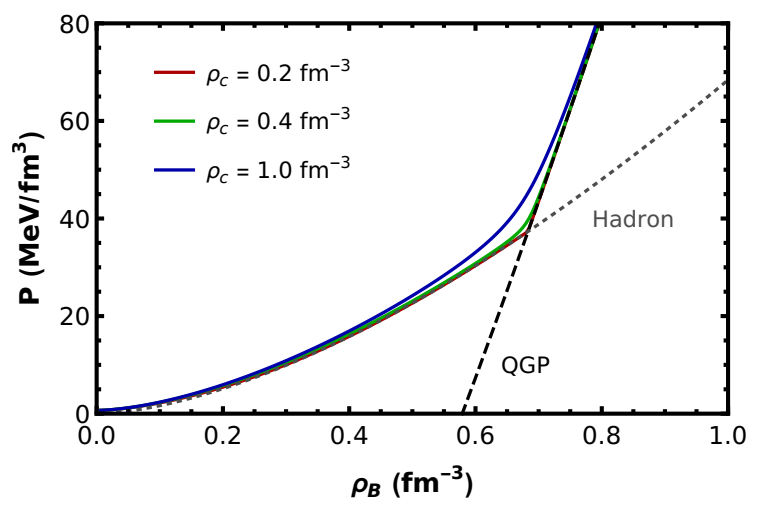

Figura 5.1: Pressão em função da densidade bariônica com o valor de $\delta_{0}=$ $100\left(\mathrm{MeV} / \mathrm{fm}^{3}\right)^{2}$ para diferentes valores de $\rho_{c}$.

bariônica, as equações de estado híbridas (linhas contínuas) adquirem o mesmo comportamento da equação de estado hadrônica (linha pontilhada). Já na região de alta densidade bariônica, as equações híbridas reproduzem a equação de estado de quarks (linha tracejada). Assim, podemos dizer que, para valores pequenos de $\rho_{B}$, a matéria se encontra na fase de hádrons e, para valores altos, a matéria se encontra na fase de quarks. A diferença entre as equações híbridas se dá apenas na região em que ocorre a intersecção entre as EoS de quarks e de hádrons (ou, fisicamente, no ponto de transição de fase). Valores maiores de $\rho_{c}$ suavizam a curva nessa região de transição, reproduzindo assim a existência de uma fase mista. Dessa forma, vemos que, para $\rho_{c}=1.0 \mathrm{fm}^{-3}$, a fase mista deve existir 
na região de $0.4 \mathrm{fm}^{-3} \lesssim \rho_{B} \lesssim 0.75 \mathrm{fm}^{-3}$; para $\rho_{c}=0.4 \mathrm{fm}^{-3}$, a fase mista diminui, situando-se na região de $0.55 \mathrm{fm}^{-3} \lesssim \rho_{B} \lesssim 0.7 \mathrm{fm}^{-3}$; para $\rho_{c}=0.2 \mathrm{fm}^{-3}$, praticamente não há fase mista.

Por outro lado, fixando o valor de $\rho_{c}=0.5 \mathrm{fm}^{-3}$ e tomando os valores de $\delta_{0}=0,100$, $500\left(\mathrm{MeV} / \mathrm{fm}^{3}\right)^{2}$, obtemos as equações de estado apresentadas na figura 5.2. Nesta figura,

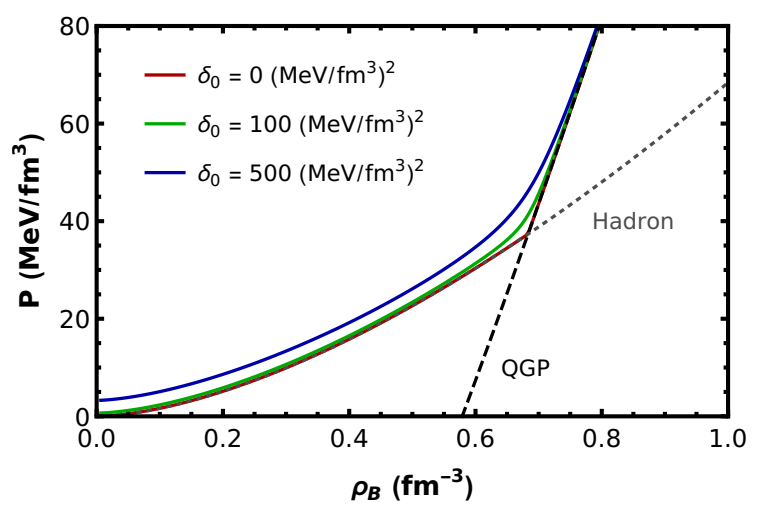

Figura 5.2: Pressão em função da densidade bariônica com o valor de $\rho_{c}=0.5 \mathrm{fm}^{-3}$ para diferentes valores de $\delta_{0}$.

vemos que o efeito causado pelo parâmetro $\delta_{0}$ é semelhante ao causado pelo parâmetro $\rho_{c}$ : com valores mais altos de $\delta_{0}$, obtemos uma transição de fase mais suave. Entretanto, o aumento no valor de $\delta_{0}$ também implica numa alteração na região da fase de hádrons. Nessa região de baixa densidade, a EoS híbrida adquire valores de pressão mais altos do que a EoS de hádrons, que é um comportamento que não desejamos. Por outro lado, valores muito baixos de $\delta_{0}$ não permitem gerar uma região de fase mista grande, mesmo com valores maiores de $\rho_{c}$. Com isso, fixamos o valor de $\delta_{0}$ em um valor intermediário, sendo

$$
\delta_{0}=100\left(\mathrm{MeV} / \mathrm{fm}^{3}\right)^{2} .
$$

Este valor nos permite modificar a forma da transição de fase através do parâmetro $\rho_{c}$ sem perder qualidade na reprodução da fase de hádrons. Para o parâmetro $\rho_{c}$, tomamos os valores sendo

$$
\rho_{c}=0.2,0.4,1.0 \mathrm{fm}^{-3} \text {. }
$$

Feito isso, podemos fixar um valor de $\rho_{B}$ e, usando as relações (3.1.14) e (4.1.6), obtemos o valor da pressão $P$ e da densidade de energia $\epsilon$. Repetindo esse procedimento para diferentes valores de $\rho_{B}$, obtemos as equações de estado apresentadas em linhas contínuas nas figuras 5.3 e 5.4 .

O gráfico da esquerda na figura 5.3 é o mesmo da figura 5.1, porém com enfoque na região de transição de fase. Nestes três gráficos, podemos notar o que já foi observado anteriormente: quanto menor é o valor de $\rho_{c}$, mais abrupta a transição de fase se torna. 

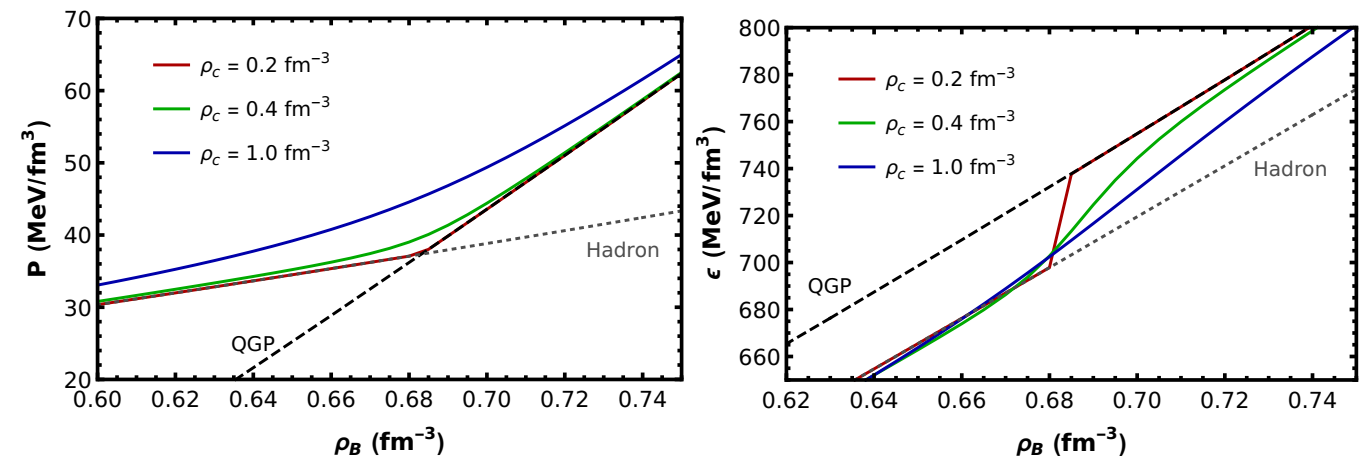

Figura 5.3: Pressão (à esquerda) e densidade de energia (à direita) em função da densidade bariônica. As linhas contínuas representam as equações híbridas MIT + Fermi com os valores de $\rho_{c}=0.2$, em vermelho; $\rho_{c}=0.4$, em verde; $\rho_{c}=1.0$, em azul. A linha tracejada representa a equação de estado do modelo de sacola do MIT, enquanto que a linha pontilhada, o modelo de gás de fermi.

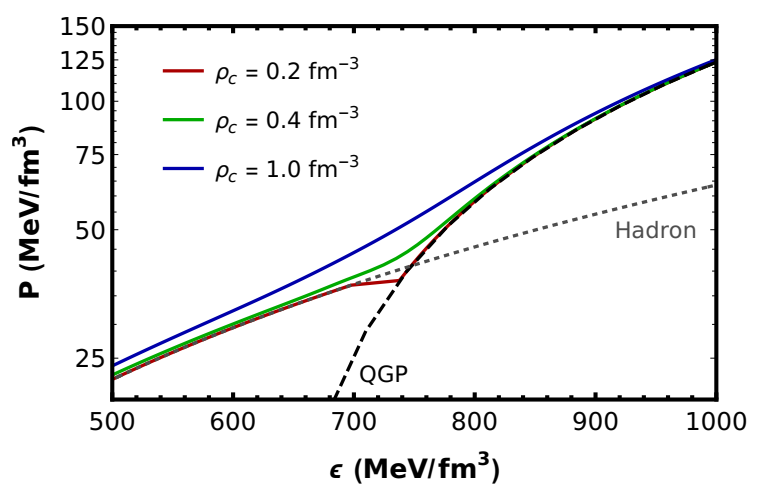

Figura 5.4: Pressão em função da densidade de energia. As linhas contínuas representam as equações híbridas MIT + Fermi, com os valores de $\rho_{c}=0.2$, em vermelho; $\rho_{c}=0.4$, em verde; $\rho_{c}=1.0$, em azul. A linha tracejada representa a equação de estado do modelo de sacola do MIT, enquanto que a linha pontilhada, o modelo de gás de Fermi.

Para verificar as consequências do tamanho da região de fase mista nas estrelas de nêutrons, resolvemos as equações de TOV usando as equações de estado híbridas obtidas acima. Os resultados são apresentados nas figuras 5.5.

Analisando o diagrama massa-raio (figura 5.5, à esquerda), vemos que as curvas das estrelas híbridas (linhas contínuas) apresentam um comportamento mais próximo das estrelas de quarks (linha tracejada) nos pontos de raio menor, e um comportamento mais próximo das estrelas de hádrons (linha pontilhada) nos pontos de raio maior. Esse comportamento é coerente, já que o valor da pressão central das estrelas híbridas diminui da esquerda para a direita. Desta forma, as estrelas híbridas mais à esquerda da curva de massa-raio são formada majoritariamente por matéria de quarks (já que a pressão central é maior), enquanto que as mais a direita, são formadas majoritariamente por matéria de 

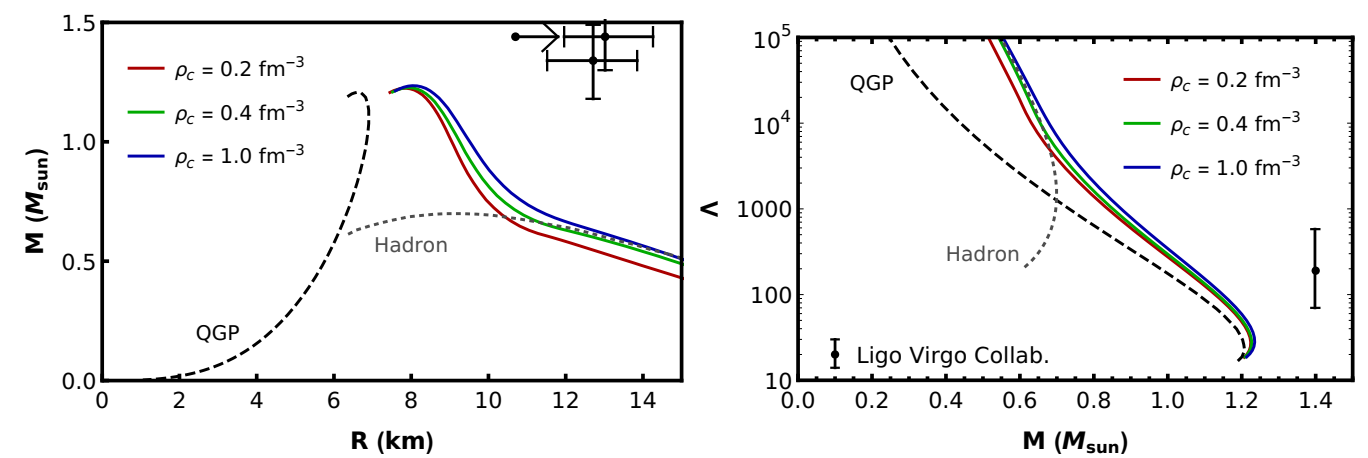

Figura 5.5: Diagrama massa-raio (à esquerda) e deformabilidade de maré em função da massa da estrela (à direita) para uma estrela de híbrida cujos constituintes são descritos pela equação de estado MIT + Fermi.

hádrons. O mesmo vale para o gráfico da deformabilidade de maré (figura 5.5, à direita).

Podemos notar também que as estrelas híbridas aparentam conectar o gráfico das estrelas de quarks com a das estrelas de hádrons, de maneira que quanto maior é o valor de $\rho_{c}$ (ou seja, quanto mais suave a transição de fase se apresenta), mais suave essa conexão é. Como consequência, na região em que ocorre um rápido aumento de massa no diagrama massa-raio, há um aumento no valor dos raios das curvas com menor valor de $\rho_{c}$.

Além disso, notamos que nenhuma das curvas reproduz os dados experimentais de forma satisfatória. Entretanto, esse resultado também é coerente, já que os modelos das fases de quarks e hádrons escolhidas não se mostraram bons modelos para estrelas de quarks e de hádrons. Para obter resultados mais compatíveis com os dados experimentais, na próxima seção escolheremos as equações da MFTQCD e de HLPS, já que foram equações que tiveram bom êxito na comparação com os dados experimentais.

\subsubsection{Modelo MFTQCD + HLPS}

Nesta seção, escolhemos as equações da MFTQCD para a fase de quarks, e as equações de HLPS para a fase de hádrons. Usaremos os três conjuntos de parâmetros da MFTQCD dados pela tabela 3.2 (equações set I, set II e set III) e os três conjuntos de parâmetros do HLPS (equações soft, intermediate e stiff). Fazendo as possíveis combinações entre essas equações, obtemos os gráficos apresentados na figura 5.6.

Neste ponto é preciso fazer uma observação. As EoS MFTQCD e HLPS foram criadas para descrever estrelas com uma única fase. Elas não "sabem" que existe outra fase da matéria e seu único compromisso é descrever os dados experimentais. Assim, é de se esperar que elas deixem de valer em algum valor de densidade. Mas esta informação deve ser introduzida de maneira "ad-hoc" (ou, com a mão). Do contrário, vamos ter 
uma EoS de hádrons fornecendo valores para a pressão em densidades altíssimas, onde não acreditamos que haja uma fase de hádrons. Da mesma forma teremos a EoS de quarks dando resultados a densidades da ordem da densidade da matéria nuclear, onde não esperamos encontrar uma fase de quarks. No que se segue, vamos adotar uma prescrição para "desligar" a EoS que produza resultados indesejados, já fora do seu domínio de validade.
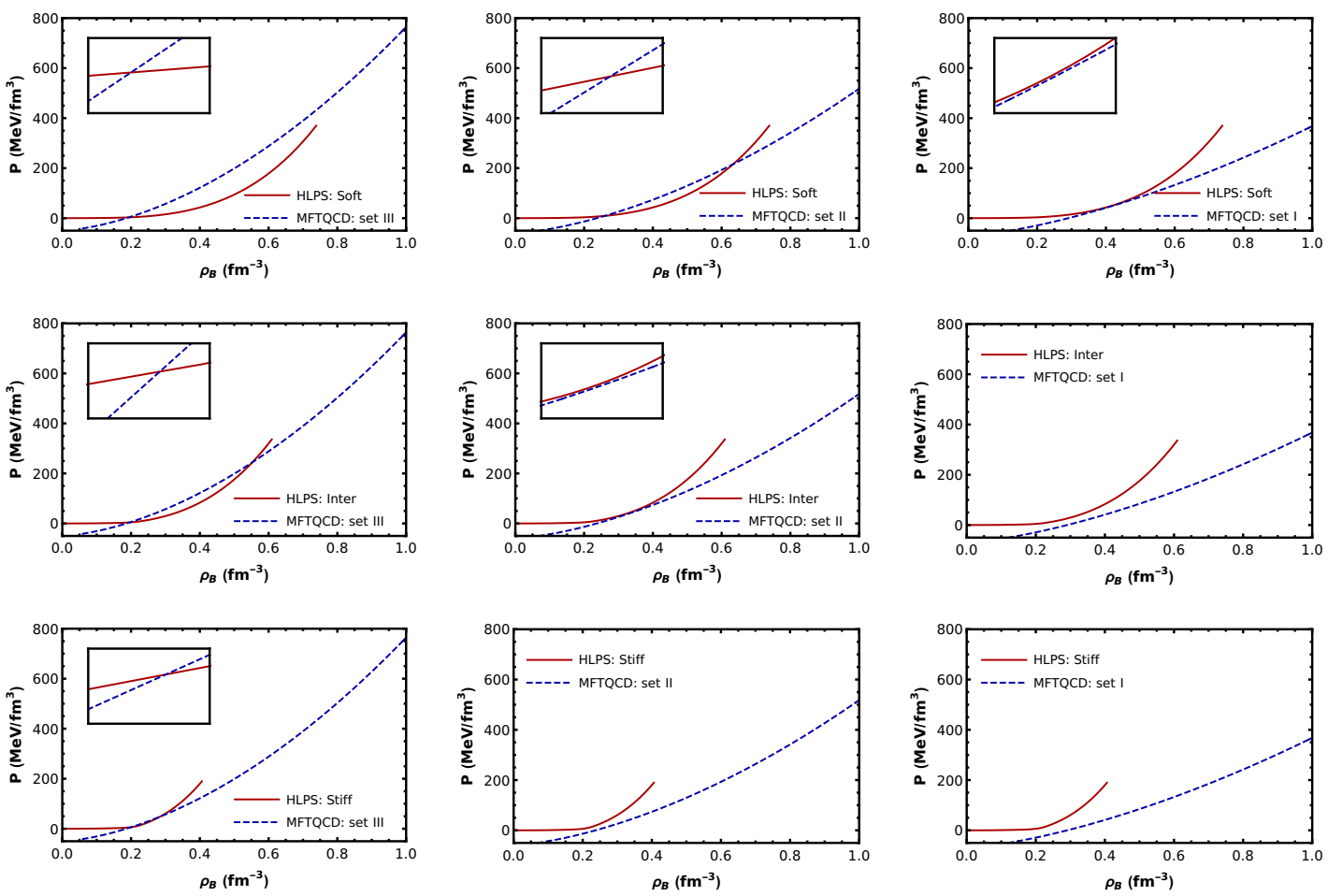

Figura 5.6: Pressão em função da densidade bariônica. Na primeira linha, temos os gráficos com o HLPS Soft; na segunda, com o HLPS Inter; na terceita, com o HLPS Stiff. Na primeira coluna, temos os gráficos com a MFTQCD set III; na segunda, com o set II; na terceira, com o set I.

$\mathrm{Na}$ figura 5.6, vemos que em apenas quatro combinações ocorre a intersecção entre as equações da fase de hádrons com a da fase de quarks. Isso implica que apenas nesta combinações pode ocorrer a transição de fase. Além disso, vemos que a equação de estado de HLPS cresce rapidamente. Isso gera dois problemas: o primeiro é que o valor da densidade bariônica em que ocorre a transição da fase de hádrons para a fase de quarks é muito baixo, sendo esses valores $\approx 0.2 \mathrm{fm}^{-3}$ (ver tabela 5.1). O segundo problema é que, por conta desse rápido crescimento, existem dois pontos de intersecção entre as funções de HLPS e MFTQCD. Fisicamente, isso representa a existência de duas transições de fase: a primeira, da fase de hádrons para a fase de quarks; e a segunda, da fase de quarks de volta para a fase de hádrons. Como não é esperado que, em densidades altas, a matéria de quarks volte para a fase de hádrons, precisamos "cortar" a EoS HLPS. Para um valor 


\begin{tabular}{|c|ccc|}
\hline & set III & set II & set I \\
\hline Soft & 0.193 & 0.260 & - \\
Inter & 0.196 & - & - \\
Stiff & 0.200 & - & - \\
\hline
\end{tabular}

Tabela 5.1: Densidade bariônica em que ocorre a transição de fase para cada combinação em $\mathrm{fm}^{-3}$.

de $\rho_{\text {corte }}$ dentro da região entre as intersecções das funções, dizemos que a EoS da fase de hádrons é tal que

- para $\rho_{B}<\rho_{\text {corte }}$, é dada pela EoS de HLPS;

- para $\rho_{B}>\rho_{\text {corte }}$, é nula.

Desta forma, escolhemos os valores de $\rho_{\text {corte }}$ sendo

- Soft-III: $\rho_{\text {corte }}=0.40 \mathrm{fm}^{-3}$,

- Inter-III: $\rho_{\text {corte }}=0.30 \mathrm{fm}^{-3}$,

- Stiff-III: $\rho_{\text {corte }}=0.24 \mathrm{fm}^{-3}$,

- Soft-II: $\rho_{\text {corte }}=0.40 \mathrm{fm}^{-3}$,

onde "Soft-III" é a equação de estado híbrida com a EoS de hádrons sendo a HLPS soft e a EoS da fase de quarks, sendo a MFTQCD set III e analogamente para os demais nomes. Fixando o valor de $\delta_{0}=40\left(\mathrm{MeV} / \mathrm{fm}^{3}\right)^{2}$, e tomando os valores de

$$
\rho_{c}=0.1,0.2,1.0 \mathrm{fm}^{-3},
$$

geramos as equações de estado mostradas nas figuras 5.7 e 5.8 .

No gráfico $p \times \epsilon$ da equação de estado Soft-II (figura 5.8, embaixo à direita), vemos que a equação de estado híbrida com o valor de $\rho_{c}=0.1 \mathrm{fm}^{-3}$ gera um crescimento muito rápido da pressão na região da transição de fase, o que implica em um comportamente não-causal $\left(c_{s}^{2}=d P / d \epsilon>1\right)$. Do que foi visto até agora, concluímos que há duas combinações que apresentam equações de estado híbridas fisicamente possíveis: Soft-III e Inter-III. Resolvendo as equações de TOV com estas duas EoS, obtemos o diagrama massa-raio e a deformabilidade de maré, apresentados nas figuras 5.9 para as equações Soft-III e 5.10 para Inter-III.

Nas figuras do diagrama de massa-raio, vemos que curvas se aproximam dos pontos dos dados experimentais. Além disso, podemos notar que os gráficos obtidos com $\rho_{c}=$ $0.1 \mathrm{fm}^{-3}$, ou seja, que apresentam uma transição de fase mais abrupta, satisfazem melhor 

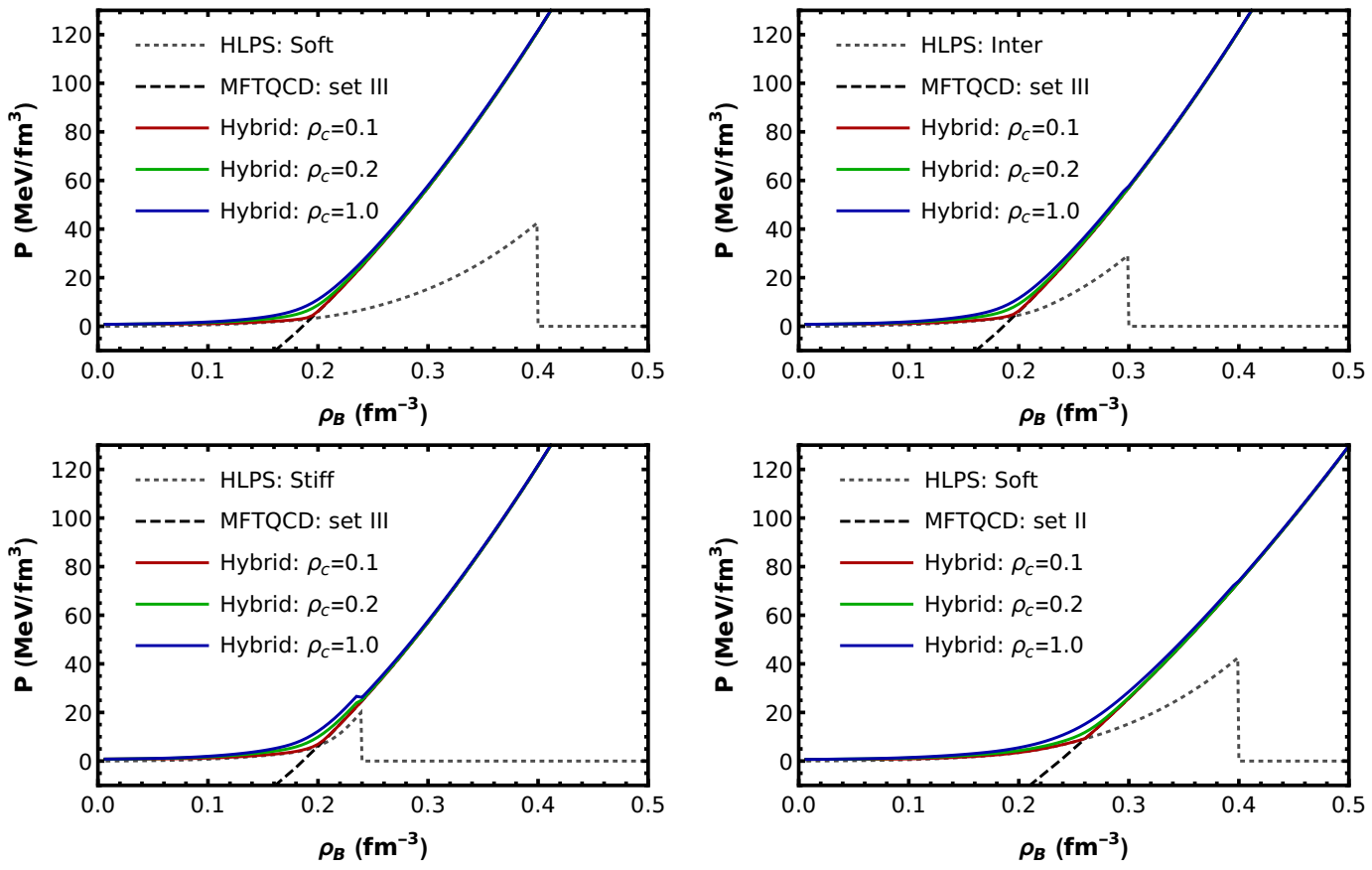

Figura 5.7: Pressão em função da densidade bariônica do modelo MFTQCD + HLPS.
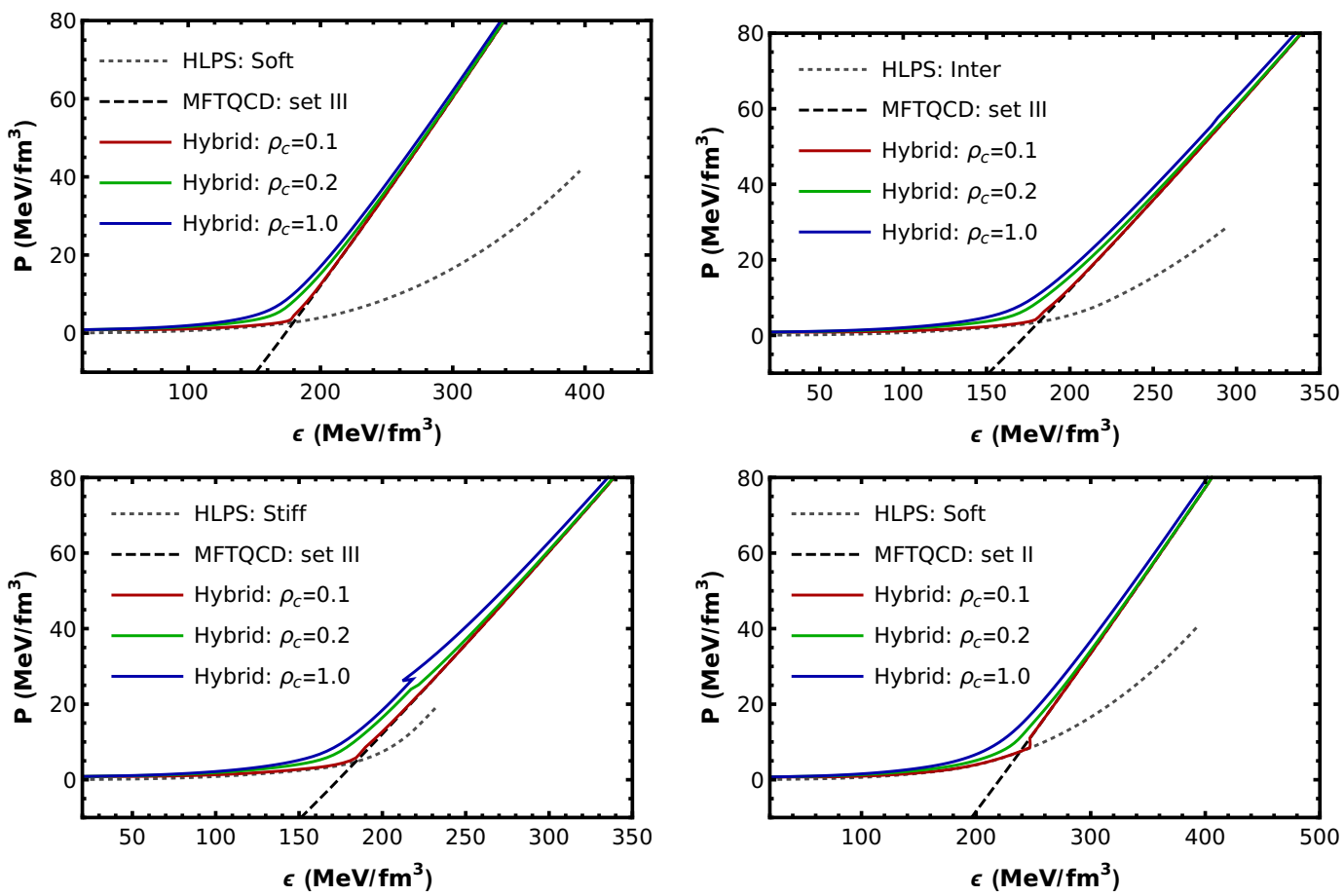

Figura 5.8: Pressão em função da densidade de energia do modelo MFTQCD + HLPS.

as restrições experimentais. O mesmo acontece para a deformabilidade de maré. Apesar de, nesse caso, os gráficos não descreverem de forma tão satisfatória o valor experimental de $\Lambda_{1.4 M_{\odot}}$, vemos que a curva com a transição de fase mais abrupta possui um valor mais próximo do dado experimental. Com isso, concluímos que, usando as equações da 

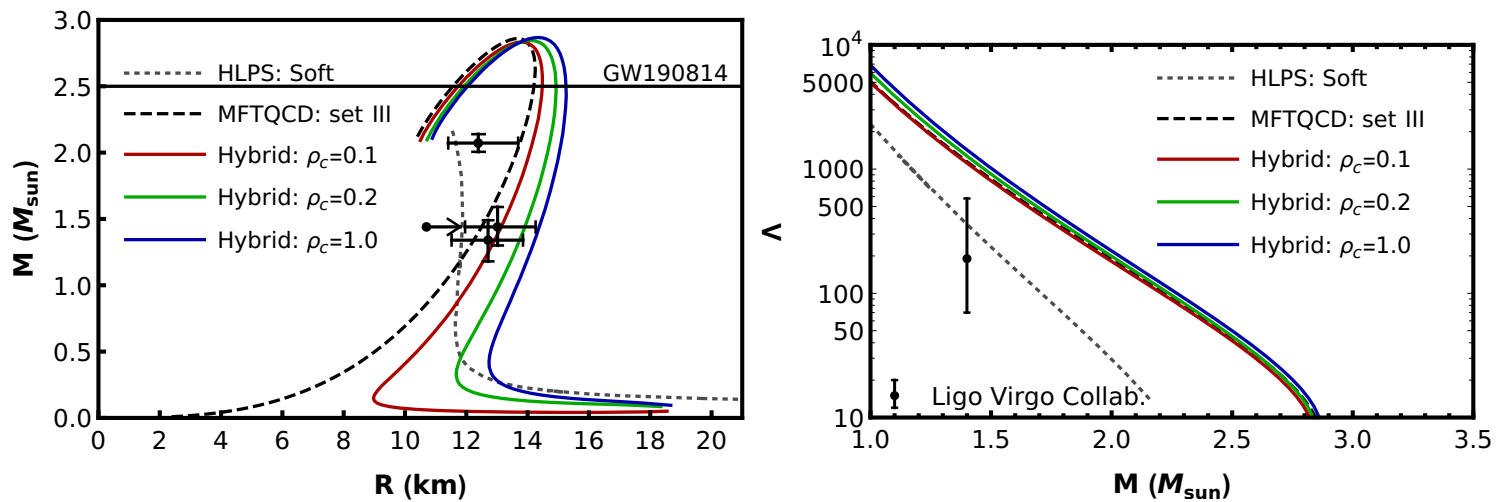

Figura 5.9: Diagrama massa-raio (à esquerda) e deformabilidade de maré em função da massa da estrela (à direita) para uma estrela de híbrida cujos constituintes são descritos pela equação de estado MFTQCD + HLPS, com o conjunto III da equação da MFTQCD e a equação soft de HLPS.
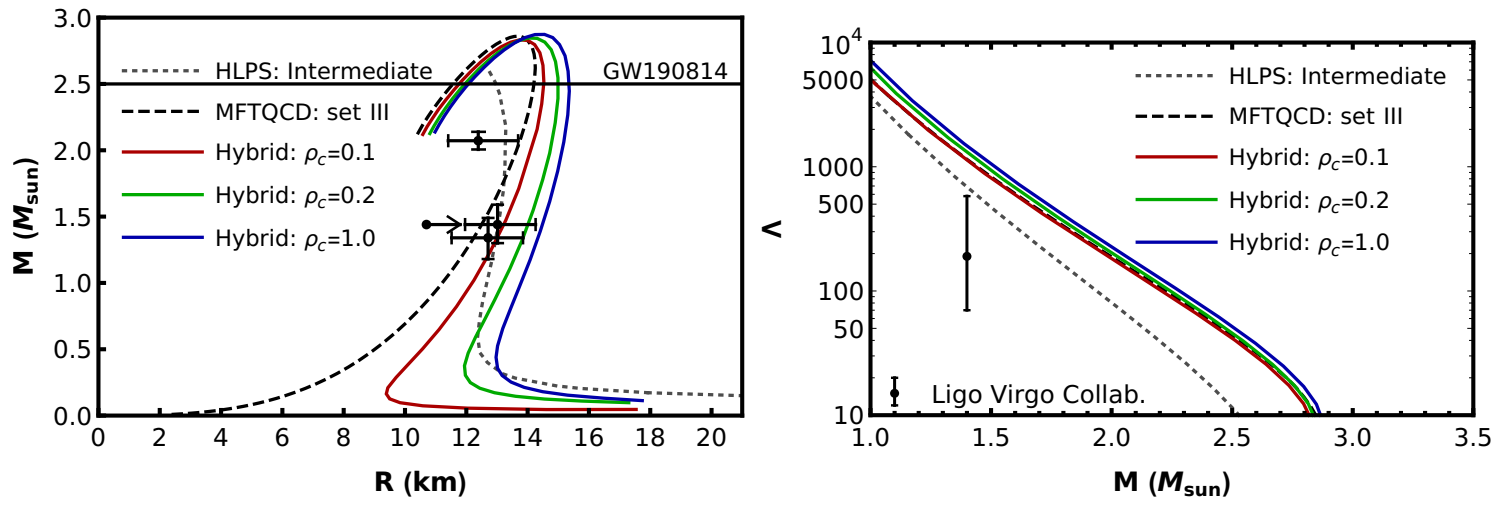

Figura 5.10: Diagrama massa-raio (à esquerda) e deformabilidade de maré em função da massa da estrela (à direita) para uma estrela de híbrida cujos constituintes são descritos pela equação de estado MFTQCD + HLPS, com o conjunto III da equação da MFTQCD e a equação intermediate de HLPS.

MFTQCD para a fase de quarks e o modelo de HLPS para a fase de hádrons, o tipo de transição de fase mais provável é uma transição abrupta, com menores valores de $\rho_{c}$.

Podemos ver também que os resultados das equações Soft-III e Inter-III são bem parecidos. Isto acontece porque a diferença entre essas equações vem apenas da EoS da fase de hádrons. Como a transição de fase ocorre em um valor de $\rho_{B}$ pequeno em ambos os casos, a matéria se apresenta na fase de quarks para um intervalo grande de densidade bariônica. Assim, as EoS Soft-III e Inter-III são idênticas na maior parte dos valores de $\rho_{B}$

Apesar dos resultados indicarem que uma transição de fase de $1^{\mathrm{a}}$ ordem gera curvas mais próximas dos dados experimentais, não podemos ignorar os problemas que surgiram nesta seção. O primeiro é que parece existir uma certa incompatibilidade entre as equa- 
ções de estado de MFTQCD e as equações de estado de HLPS. Das nove combinações mostradas na figura 5.6, apenas duas geram uma equação de estado híbrida fisicamente coerente. Além disso, como já apontado anteriormente, os valores de densidade bariônica nos quais ocorre a transição de fase são valores muito baixos, próximos de $\approx 0.2 \mathrm{fm}^{-3}$, que é apenas um pouco maior do que a densidade de saturação nuclear $\left(\rho_{B}=0.16 \mathrm{fm}^{-3}\right)$. Usando as EoS de MFTQCD + HLPS com os parâmetros usados nesta seção chegaríamos à conclusão de que existem quarks desconfinados em densidades da ordem da densidade de saturação nuclear, o que é muito difícil de aceitar. Assim, para obter resultados plausíveis, seria necessário mudar os valores dos parâmetros utilizados nos modelos, ou até mesmo mudar os modelos das fases de quarks e/ou de hádrons.

$\mathrm{Na}$ literatura, encontramos apenas um trabalho, publicado em [17], onde os autores investigaram o impacto da natureza da transição de fase sobre as propriedades das estrelas híbridas. Eles usaram outros modelos para as fases hadrônicas e de quarks. Além disso, eles usaram outra equação, diferente de (5.1.1), para conectar as duas fases. Ao invés do parâmetro $\rho_{c}$, eles controlam a densidade na qual ocorre a transição de fase $\left(n_{\text {trans }}\right.$, em função de $n_{0}$ a densidade de saturação da matéria nuclear) e o salto na densidade de energia durante a transição $(\Delta \epsilon)$. Na figura 5.11, apresentamos os resultados obtidos em [17]. As curvas em preto representam as estrelas de nêutrons contituídas unicamente por hádrons. As linhas coloridas representam estrelas híbridas com diferentes parâmetros. Observamos que à medida em que $\Delta \epsilon$ diminui (transição mais suave), as curvas se deslocam para a direita e para cima, exatamente como as da nossa figura 5.10. Vemos, porém, que na figura 5.11, este deslocamento é muito mais pronunciado. Em todos os casos, as densidades de transição de fase estão entre 2 e $3 n_{0}$. A exceção é a curva cinza, onde a transição de fase ocorre em $n_{0}$, como nos casos considerados na nossa figura 5.10. A curva cinza tem um aspecto (de " $\mathrm{S}$ " invertido) semelhante às nossas, porém o valor da massa máxima e o raio associado a ela são bem diferentes.

Concluímos que o comportamento que obtemos é o comportamento esperado para o caso de uma transição de fase ocorrendo em valores baixos de densidade bariônica. $\mathrm{O}$ nosso modelo híbrido produz resultados compatíveis com os da referência [17]. Nos gráficos da figura 5.11, vemos que na região de baixa densidade central, todas as estrelas híbridas apresentam o mesmo comportamento da estrela pura de hádrons. Nos nossos resultados, existe apenas uma aproximação desse comportamento. Em outras palavras, as linhas das estrelas híbridas não se juntam totalmente sobre a linha da estrela de hádrons. Isso pode ser uma indicação de que o nosso modelo de estrelas híbridas não produz resultados muito confiáveis na região de baixa pressão central, devido à equação usadas para a fase mista.

Com isso, vemos que a análise proposta nesta seção pode, em princípio, extrair a 

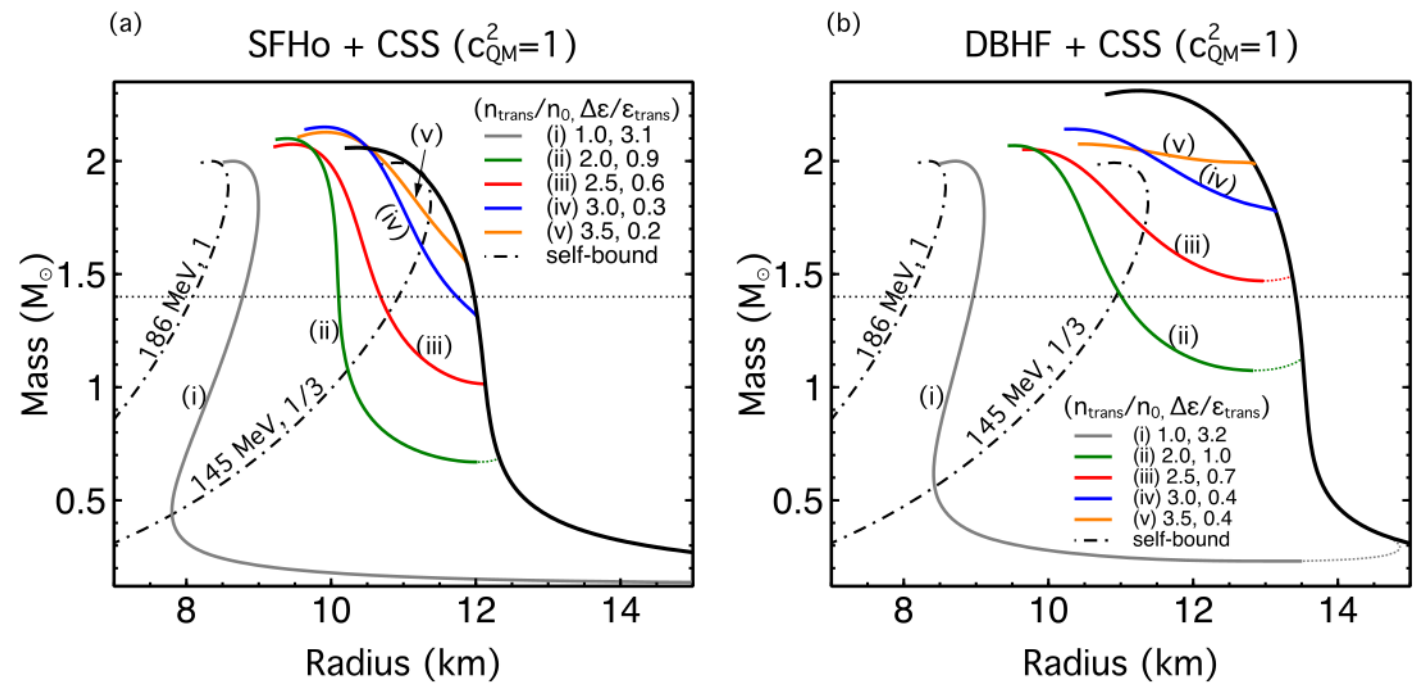

Figura 5.11: Diagrama massa-raio do artigo [17]. A linha preta contínua representa a estrela de nêutrons constituída inteiramente por hádrons e as linhas coloridas, são as estrelas híbridas com diferentes escolhas de parâmetros.

forma da transição de fase hádron-quark a partir dos dados experimentais. Porém, antes disso, é necessário realizar um estudo mais aprofundado. O próximo passo será considerar que os parâmetros $\xi$ e $B$ da MFTQCD dependam de $\rho_{B}$. Com isso, podemos ajustar a equação de estado da MFTQCD para que tenha valores menores de pressão na região de menor densidade. Assim, a transição de fase irá ocorrer em valores maiores de densidade bariônica. Além disso será necessário testar outros modelos. 


\section{CAPÍtulo}

\section{Conclusão}

A matéria das estrelas de nêutrons, que se encontra em baixas temperaturas e em altas densidades, está numa região pouco compreendida do diagrama de fase da QCD. Nesta região, não podemos contar com os resultados dos cálculos de QCD na rede e precisamos usar modelos. Para testar estes modelos, podemos estudar estrelas de nêutrons e calcular quantidades que podem ser comparadas com dados experimentais. Além disso, nessa mesma região, diversos cálculos indicam que a matéria poderia desconfinar no que chamamos de matéria de quarks ou QGP frio. Assim, existem três possíveis maneiras de descrever uma estrela de nêutrons. A primeira seria considerando que a estrela é constituída inteiramente por quarks (estrelas de quarks), a segunda, considerando que a estrela é constituída inteiramente por hádrons (estrelas de hádrons), e a última, considerando que no "caroço" da estrela a matéria se encontra na fase de QGP, e na parte mais externa a matéria se encontra na fase de hádrons (estrelas híbridas).

Por conta do rápido avanço na área experimental, mais dados observacionais foram obtidos ao longo desta última década, de forma que, agora, menos modelos satisfazem as restrições impostas por esses dados.

O primeiro objetivo deste trabalho foi atualizar a MFTQCD, introduzida em [36] e aplicada a estrelas de nêutrons em [12]. Modelamos estrelas de quarks a partir da MFTQCD e obtivémos o diagrama massa-raio e a deformabilidade de maré. Comparando com os dados experimentais atuais, concluímos que a MFTQCD continua sendo uma boa aproximação para descrever o QGP frio. Entretanto, o conjunto dos possíveis valores dos parâmetros livres se tornou mais restrito.

O segundo objetivo foi estudar qual é o impacto da natureza da transição de fase sobre as características das estrelas de nêutrons. Tentamos determinar se esta transição é mais próxima de uma transição de $1^{\mathrm{a}}$ ordem ou de um cross-over. Escolhendo um modelo para a fase de quarks e outro para a fase de hádrons, usamos uma equação de estado [37] que contém uma fase mista na vizinhança de transição de fase. Fixando um modelo para cada 
fase e variando o parâmetro livre $\rho_{c}$, obtemos equações de estado com transições de fase de diferentes intensidades. Primeiramente, com o objetivo de estudar o comportamento esquemático das equações de estado, escolhemos os modelos mais simples: o modelo de sacola do MIT para descrever a fase de quarks e o modelo do gás de Fermi para a fase de hádrons. Em seguida, para obter um resultado mais próximo dos dados experimentais, escolhemos a MFTQCD para a fase de quarks e o modelo de HLPS para a fase de hádrons. Tomamos três equações de estado com diferentes valores de $\rho_{c}$, sendo a primeira mais próxima de uma transição de $1^{\mathrm{a}}$ ordem $\left(\rho_{c}=0.1 \mathrm{fm}^{-3}\right)$, a segunda com uma transição de fase menos abrupta $\left(\rho_{c}=0.2 \mathrm{fm}^{-3}\right)$, e a terceira reproduzindo a transição mais suave possível $\left(\rho_{c}=1.0 \mathrm{fm}^{-3}\right)$. Resolvendo as equações de TOV, concluímos que a equação de estado que melhor reproduz os dados experimentais é a que possui o valor de $\rho_{c}=0.1 \mathrm{fm}^{-3}$; ou seja, a que contém uma transição de fase mais abrupta. Entretanto, ao longo do processo para a obtenção dos resultados das equações de MFTQCD + HLPS, encontramos algumas irregularidades, como o fato de a transição de fase ocorrer em valores muito baixos de densidade bariônica $\left(\approx 0.2 \mathrm{fm}^{-3}\right)$. Com isso, esta é apenas uma conclusão provisória. Em um trabalho futuro, iremos fazer ajustes nas equações utilizadas e verificar se essa conclusão continua válida. 


\section{Dedução da TOV}

\section{A.1 Equação da Massa}

Para determinar a equação de TOV que relaciona a variação da massa pelo raio da estrela, partimos da seguinte relação

$$
d M=\rho(r) d V
$$

onde $d M$ e $d V$ são elementos infinitesimais da massa e do volume da estrela respectivamente, enquanto $\rho(r)$ é a sua densidade de massa. Da relatividade restrita, podemos relacionar a massa e a energia, de forma que

$$
\epsilon(r)=\rho(r) c^{2}=\rho(r)
$$

onde $\epsilon(r)$ é a densidade de energia, e na segunda igualmente estamos usando unidades naturais $(c=1)$. Além disso, supondo que a estrela é esférica, o elemento infinitesimal de volume pode ser escrito como

$$
d V=4 \pi r^{2} d r
$$

Dessa forma, obtemos que

$$
d M=4 \pi r^{2} \epsilon(r) d r
$$

e portanto,

$$
\frac{d M}{d r}=4 \pi r^{2} \epsilon(r)
$$

que é a equação de TOV para a massa da estrela. 


\section{A.2 Equação da Pressão}

A equação de TOV que relaciona a variação da pressão pelo raio da estrela é obtida através da equação de Einstein,

$$
G_{\nu}^{\mu}=-8 \pi G T_{\nu}^{\mu},
$$

onde $G_{\nu}^{\mu}$ é o tensor de curvatura de Einstein e $T_{\nu}^{\mu}$, o tensor energia-momento.

Primeiramente, para obter $G_{\nu}^{\mu}$, precisamos da métrica da estrela. Supondo que a estrela seja esférica e estática, o seu elemento de linha é dado por

$$
d s^{2}=e^{2 \nu(r)} d t^{2}-e^{2 \lambda(r)} d r^{2}-r^{2}\left[d \theta^{2}+\sin ^{2} \theta d \phi^{2}\right] .
$$

Sabendo que $d s^{2}=g_{\mu \nu} d x^{\mu} d x^{\nu}$, então os elementos da métrica dessa estrela vão ser

$$
g^{00}=e^{2 \nu(r)}, \quad g^{11}=-e^{2 \lambda(r)}, \quad g^{22}=-r^{2}, \quad g^{33}=-r^{2} \sin ^{2} \theta,
$$

com os demais termos sendo nulos. Além disso, como devemos ter $g_{\mu \nu} g^{\nu \rho}=\delta_{\mu}^{\rho}$, então

$$
g^{00}=e^{-2 \nu(r)}, \quad g^{11}=-e^{-2 \lambda(r)}, \quad g^{22}=-\frac{1}{r^{2}}, \quad g^{33}=-\frac{1}{r^{2} \sin ^{2} \theta} .
$$

Sabemos os elementos da métrica, calculamos os símbolos de Cristoffel, dado por

$$
\Gamma_{\mu \nu}^{\rho}=\frac{1}{2} g^{\rho \alpha}\left(\partial_{\mu} g_{\alpha \nu}+\partial_{\nu} g_{\mu \alpha}-\partial_{\alpha} g_{\mu \nu}\right)
$$

Note que, como $g_{\mu \nu}$ é diagonal, então da somatória em $\alpha$ sobra apenas o termo em que $\alpha=\rho$. Dessa forma, os símbolos de Cristoffel são reduzidos para

$$
\Gamma_{\mu \nu}^{\rho}=\frac{1}{2} g^{\rho \rho}\left(\partial_{\mu} g_{\rho \nu}+\partial_{\nu} g_{\mu \rho}-\partial_{\rho} g_{\mu \nu}\right)
$$

Para facilitar os cálculos, podemos notar que, para $\mu \neq \nu \neq \rho, \Gamma_{\mu \nu}^{\rho}=0$, devido ao fato de $g_{\mu \nu}$ ser diagonal. Além disso, podemos usar a propriedade de que $\Gamma_{\mu \nu}^{\rho}=\Gamma_{\nu \mu}^{\rho}$. Fazendo os cálculos restantes, obtemos que os símbolos de Cristoffel não nulos são

$$
\begin{aligned}
& \Gamma_{01}^{0}=\Gamma_{10}^{0}=\nu^{\prime}(r), \\
& \Gamma_{00}^{1}=\nu^{\prime}(r) e^{2[\nu(r)-\lambda(r)]}, \quad \Gamma_{11}^{1}=\lambda^{\prime}(r), \quad \Gamma_{22}^{1}=-r e^{-\lambda(r)}, \\
& \Gamma_{33}^{1}=-r \sin ^{2} \theta e^{-2 \lambda(r)}, \quad \Gamma_{12}^{1}=\Gamma_{21}^{1}=\frac{1}{r}, \\
& \Gamma_{33}^{2}=-\sin \cos \theta, \\
& \Gamma_{13}^{3}=\Gamma_{31}^{3}=\frac{1}{r}, \quad \Gamma_{23}^{3}=\Gamma_{32}^{3}=\cot \theta .
\end{aligned}
$$

Com isso, conseguimos calcular o tensor de Ricci,

$$
R_{\mu \nu}=R_{\mu \nu \rho}^{\rho}
$$


onde $R_{\mu \nu \sigma}^{\rho}$ é o tensor de Riemann

$$
R_{\mu \nu \sigma}^{\rho}=\partial_{\nu} \Gamma_{\mu \sigma}^{\rho}-\partial_{\sigma} \Gamma_{\mu \nu}^{\rho}+\Gamma_{\mu \sigma}^{\alpha} \Gamma_{\alpha \nu}^{\rho}-\Gamma_{\mu \nu}^{\alpha} \Gamma_{\alpha \sigma}^{\rho}
$$

Como vamos precisar apenas dos tensores de Riemann em que $\sigma=\rho$, é possível demonstrar, usando os símbolos de Cristoffel calculados da equação (A.2.7), que ${ }^{1}$

$$
R_{\mu \nu}=R_{\mu \nu \rho}^{\rho}=0, \text { se } \mu \neq \nu \text {. }
$$

Com isso, apenas quatro componentes do tensor de Ricci não são nulas. Calculando-as, obtemos que

$$
\begin{aligned}
& R_{00}=\left[-\nu^{\prime \prime}(r)-\nu^{\prime 2}(r)+\nu^{\prime}(r) \lambda^{\prime}(r)-2 \frac{\nu^{\prime}(r)}{r}\right] e^{2[\nu(r)-\lambda(r)]}, \\
& R_{11}=\nu^{\prime \prime}(r)+\nu^{\prime 2}(r)-\nu^{\prime}(r) \lambda^{\prime}(r)-2 \frac{\nu^{\prime}(r)}{r}, \\
& R_{22}=\left[r \nu^{\prime}(r)-r \lambda^{\prime}(r)+1\right] e^{-2 \lambda(r)}-1, \\
& R_{33}=\left[\left(r \nu^{\prime}(r)-r \lambda^{\prime}(r)+1\right) e^{-2 \lambda(r)}-1\right] \sin ^{2} \theta .
\end{aligned}
$$

Com esses valores, conseguimos calcular o escalar de Ricci,

$$
R=g^{\mu \nu} R_{\mu \nu}
$$

que resulta em

$$
R=2\left[-\nu^{\prime \prime}(r)-\nu^{\prime 2}(r)+\nu^{\prime}(r) \lambda^{\prime}(r)-\frac{2}{r}\left(\nu^{\prime}(r)-\lambda^{\prime}(r)\right)-\frac{1}{r^{2}}\right] e^{-2 \lambda(r)}+\frac{2}{r^{2}} .
$$

Com o tensor e escalar de Ricci calculados, podemos obter o tensor de curvatura de Einstein, dado por

$$
G_{\nu}^{\mu}=g^{\alpha \nu} R_{\mu \nu}-\frac{1}{2} \delta_{\nu}^{\mu} R
$$

Como $R_{\mu \nu}$ e $g^{\alpha \nu}$ são diagonais, então

$$
G_{\nu}^{\mu}=0, \text { se } \mu \neq \nu
$$

Dessa forma, sobram apenas quatro componentes de $G_{\nu}^{\mu}$. Calculando-os, obtemos

$$
\begin{aligned}
G_{0}^{0} & =\left[\frac{1}{r^{2}}-2 \frac{\lambda^{\prime}(r)}{r}\right] e^{-2 \lambda(r)}-\frac{1}{r^{2}}, \\
G_{1}^{1} & =\left[\frac{1}{r^{2}}+2 \frac{\nu^{\prime}(r)}{r}\right] e^{-2 \lambda(r)}-\frac{1}{r^{2}}, \\
G_{2}^{2}=G_{3}^{3} & =\left[\nu^{\prime \prime}(r)+\nu^{\prime 2}(r)-\nu^{\prime}(r) \lambda^{\prime}(r)+\frac{\nu^{\prime}(r)-\lambda^{\prime}(r)}{r}\right] e^{-2 \lambda(r)},
\end{aligned}
$$

\footnotetext{
${ }^{1}$ Uma sugestão para facilitar os cálculos é calcular os termos $R_{\mu \nu 0}^{0}, R_{\mu \nu 1}^{1}, R_{\mu \nu 2}^{2}$ e $R_{\mu \nu 3}^{3}$ para $\mu$ e $\nu$ quaisquer, desde que $\mu \neq \nu$.
} 
e assim, obtemos o lado esquerdo da equação (A.2.1). Para obter o lado direito dessa equação, precisamos calcular o tensor energia-momento, dado por

$$
T_{\nu}^{\mu}=(\epsilon+P) u^{\mu} u_{\nu}-P \delta_{\nu}^{\mu},
$$

onde $u^{\mu}$ é o quadrivetor velocidade do fluido. No referencial em que o fluido está em repouso, temos que

$$
u^{\mu}=(1,0,0,0) .
$$

Usando isso para calcular $T_{\nu}^{\mu}$, obtemos que as componentes não nulas são

$$
\begin{aligned}
& T_{0}^{0}=\epsilon, \\
& T_{i}^{i}=-P, \text { onde } i=1,2,3 .
\end{aligned}
$$

Substiuindo essas componentes de $T_{\nu}^{\mu}$ e os valores de $G_{\nu}^{\mu}$ calculados na equação (A.2.1), obtemos o sistema de equações

$$
\begin{array}{r}
{\left[\frac{1}{r^{2}}-2 \frac{\lambda^{\prime}(r)}{r}\right] e^{-2 \lambda(r)}-\frac{1}{r^{2}}=-8 \pi G \epsilon,} \\
{\left[\frac{1}{r^{2}}+2 \frac{\nu^{\prime}(r)}{r}\right] e^{-2 \lambda(r)}-\frac{1}{r^{2}}=8 \pi G P,} \\
{\left[\nu^{\prime \prime}(r)+\nu^{\prime 2}(r)-\nu^{\prime}(r) \lambda^{\prime}(r)+\frac{\nu^{\prime}(r)-\lambda^{\prime}(r)}{r}\right] e^{-2 \lambda(r)}=8 \pi G P .}
\end{array}
$$

Podemos notar que, partindo de

$$
\begin{aligned}
\frac{d}{d r}\left[r\left(1-e^{-2 \lambda(r)}\right)\right] & =1-e^{-2 \lambda(r)}+r\left(2 \lambda^{\prime}(r) e^{-2 \lambda(r)}\right) \\
& =\left[-1+2 r \lambda^{\prime}(r)\right] e^{-2 \lambda(r)}+1
\end{aligned}
$$

e multiplicando ambos os lados por $-1 / r^{2}$,

$$
-\frac{1}{r^{2}} \frac{d}{d r}\left[r\left(1-e^{-2 \lambda(r)}\right)\right]=\left[\frac{1}{r^{2}}-2 \frac{\lambda^{\prime}(r)}{r}\right] e^{-2 \lambda(r)}-\frac{1}{r^{2}},
$$

obtemos o lado esquerdo da equação (A.2.14). Substituindo esse resultado na equação (A.2.14),

$$
-\frac{1}{r^{2}} \frac{d}{d r}\left[r\left(1-e^{-2 \lambda(r)}\right)\right]=-8 \pi G \epsilon
$$

Multiplicando ambos os lados por $-r^{2}$ e integrando-os em $r$, obtemos

$$
\left[r\left(1-e^{-2 \lambda(r)}\right)\right]=8 \pi G \int_{0}^{r} d r^{\prime} r^{\prime 2} \epsilon\left(r^{\prime}\right) .
$$

Isolando o termo $e^{-2 \lambda(r)}$, temos que

$$
e^{-2 \lambda(r)}=1-\frac{8 \pi G}{r} \int_{0}^{r} d r^{\prime} r^{\prime 2} \epsilon\left(r^{\prime}\right) .
$$


Da equação (A.1.1), podemos obter que

$$
M(r)=4 \pi \int_{0}^{r} d r^{\prime} r^{\prime 2} \epsilon\left(r^{\prime}\right) .
$$

Substituindo essa integral na equação anterior, obtemos que

$$
e^{-2 \lambda(r)}=1-\frac{8 \pi G}{r} \frac{M(r)}{4 \pi}
$$

e assim,

$$
e^{2 \lambda(r)}=\left(1-\frac{2 G M(r)}{r}\right)^{-1} .
$$

Das equações (A.2.14) e (A.2.15), podemos isolar, respectivamente, $\lambda^{\prime}(r)$ e $\nu^{\prime}(r)$, e obter

$$
\begin{aligned}
& \lambda^{\prime}(r)=+\frac{1}{2 r}\left[1-\left(1-8 \pi G r^{2} \epsilon(r)\right) e^{2 \lambda(r)}\right], \\
& \nu^{\prime}(r)=-\frac{1}{2 r}\left[1-\left(1+8 \pi G r^{2} P(r)\right) e^{2 \lambda(r)}\right] .
\end{aligned}
$$

A partir dessas equações, podemos calcular os termos $\nu^{\prime \prime}(r), \nu^{\prime 2}(r)$ e $\nu^{\prime}(r) \lambda^{\prime}(r)$ e substituílos na equação (A.2.16), obtendo

$$
\begin{aligned}
& 8 \pi G r^{2} P^{2}(r) e^{2 \lambda(r)}+\left(-1+e^{2 \lambda(r)}\right) \epsilon(r) \\
& \quad+P(r)\left[-1+e^{2 \lambda(r)}+8 \pi G r^{2} \epsilon(r) e^{2 \lambda(r)}\right]+2 r P^{\prime}(r)=0 .
\end{aligned}
$$

Organizando essa equação, ficamos com

$$
[P(r)+\epsilon(r)]\left\{e^{2 \lambda(r)}\left[8 \pi G r^{2} P(r)+1\right]-1\right\}+2 r P^{\prime}(r)=0 .
$$

Substituindo a equação (A.2.17) nesta última equação e organizando os termos, obtemos a equação de TOV que relaciona a variação da pressão pelo raio da estrela

$$
\frac{d P}{d r}=-\frac{G \epsilon(r) M(r)}{r^{2}}\left[1+\frac{P(r)}{\epsilon(r)}\right]\left[1+\frac{4 \pi r^{3} P(r)}{M(r)}\right]\left[1-\frac{2 G M(r)}{r}\right]^{-1} .
$$

Por último, deduziremos a quantidade $e^{2 \nu(r)}$. Considerando a métrica no vácuo e somando a equação (A.2.18) com a equação (A.2.19), obtemos

$$
\nu^{\prime}(r)+\lambda^{\prime}(r)=0 .
$$

Integrando ambos os lados em $r$, temos que

$$
\nu(r)+\lambda(r)=c,
$$

onde $c$ é a constante de integração a ser descoberta. Por outro lado, podemos notar que $e^{2 \nu(r)}$ e $e^{2 \lambda(r)}$ devem tender a 1 em regiões distantes da estrela $\left(g_{\mu \nu}\right.$ deve tender à métrica do espaco-tempo plano). Dessa forma, devemos ter

$$
e^{2[\nu(r)+\lambda(r)]}=1 \Rightarrow \nu(r)+\lambda(r)=0, \text { para } r \rightarrow \infty .
$$


Entretanto, por conta da equação (A.2.21), concluímos que

$$
\nu(r)+\lambda(r)=0
$$

para qualquer valor de $r$. Com essa última relação, podemos escrever que

$$
e^{2 \nu(r)}=e^{-2 \lambda(r)}
$$

e usando a equação (A.2.17), obtemos

$$
e^{2 \nu(r)}=1-\frac{2 G M(r)}{r} .
$$




\section{Resolução Numérica da TOV}

Sabendo as equações de estado e os valores iniciais

$$
r_{0}=0.001 \mathrm{~km}, \quad P\left(r_{0}\right)=P_{c}, \quad M\left(r_{0}\right)=0, \quad H\left(r_{0}\right)=r_{0}^{2}, \quad \beta\left(r_{0}\right)=2 r_{0},
$$

podemos calcular o valor da densidade de energia no ponto $r_{0}$ numericamente ${ }^{1}$. Conhecendo agora os valores de $r_{0}, P\left(r_{0}\right), M\left(r_{0}\right)$ e $\epsilon\left(r_{0}\right)$, calculamos os valores de $P^{\prime}\left(r_{0}\right)$ e $M^{\prime}\left(r_{0}\right)$ a partir das equações de TOV

$$
\begin{aligned}
& \left(\frac{d P}{d r}\right)_{r_{0}}=-\frac{G \epsilon\left(r_{0}\right) M\left(r_{0}\right)}{r_{0}^{2}}\left[1+\frac{P\left(r_{0}\right)}{\epsilon\left(r_{0}\right)}\right]\left[1+\frac{4 \pi r_{0}^{3} P\left(r_{0}\right)}{M\left(r_{0}\right)}\right]\left[1-\frac{2 G M\left(r_{0}\right)}{r_{0}}\right]^{-1}, \\
& \left(\frac{d M}{d r}\right)_{r_{0}}=4 \pi r_{0}^{2} \epsilon\left(r_{0}\right) .
\end{aligned}
$$

Em seguida, fazemos a seguinte aproximação:

$$
\begin{aligned}
r_{1} & =r_{0}+\delta r \\
P\left(r_{1}\right) & =P\left(r_{0}\right)+\delta r\left(\frac{d P}{d r}\right)_{r_{0}}, \\
M\left(r_{1}\right) & =M\left(r_{0}\right)+\delta r\left(\frac{d M}{d r}\right)_{r_{0}},
\end{aligned}
$$

onde definimos $\delta r=0.001 \mathrm{~km}$ (quanto menor o valor de $\delta r$, mais preciso será o resultado; porém, mais iterações serão necessárias).

\footnotetext{
${ }^{1}$ Para isso, podemos fazer uma interpolação da tabela de dados usada para fazer o gráfico $P \times \epsilon$. Outro jeito seria, por exemplo para o caso do modelo de sacola do MIT, resolver numericamente o sistema formado pelas equações (3.1.14) e (3.1.6) tomando o valor da pressão sendo $P=P\left(r_{0}\right)$. Uma vez feito isso, obtemos os valores dos momentos de Fermi e da densidade bariônica no raio $r_{0}$. Substituindo esses valores na equação (3.1.7), conseguimos o valor de $\epsilon\left(r_{0}\right)$.
} 
Visando agora obter os valores de $\beta\left(r_{1}\right)$ e $H\left(r_{1}\right)$, calculamos os termos

$$
\begin{aligned}
\left(\frac{d H}{d r}\right)_{r_{0}}= & \beta\left(r_{0}\right) \\
\left(\frac{d \beta}{d r}\right)_{r_{0}}= & 2\left(1-\frac{2 G M\left(r_{0}\right)}{r_{0}}\right)^{-1} H\left(r_{0}\right)\left\{-2 \pi G\left[5 \epsilon\left(r_{0}\right)+9 P\left(r_{0}\right)+\frac{\epsilon\left(r_{0}\right)+P\left(r_{0}\right)}{(d P / d \epsilon)_{r_{0}}}\right]\right. \\
& \left.+\frac{3}{r_{0}^{2}}+2\left(1-\frac{2 G M\left(r_{0}\right)}{r_{0}}\right)^{-1}\left(\frac{G M\left(r_{0}\right)}{r_{0}^{2}}+4 \pi G r_{0} P\left(r_{0}\right)\right)^{2}\right\} \\
& +\frac{2 \beta\left(r_{0}\right)}{r_{0}}\left(1-\frac{2 G M\left(r_{0}\right)}{r_{0}}\right)^{-1}\left[-1+\frac{G M\left(r_{0}\right)}{r_{0}}+2 \pi G r_{0}^{2}\left(\epsilon\left(r_{0}\right)-P\left(r_{0}\right)\right)\right],
\end{aligned}
$$

onde tomamos

$$
\left(\frac{d P}{d \epsilon}\right)_{r_{0}}=\frac{P\left(r_{1}\right)-P\left(r_{0}\right)}{\epsilon\left(r_{1}\right)-\epsilon\left(r_{0}\right)}
$$

Feito isso, fazemos a aproximação

$$
\begin{aligned}
& \beta\left(r_{1}\right)=\beta\left(r_{0}\right)+\delta r\left(\frac{d \beta}{d r}\right)_{r_{0}}, \\
& H\left(r_{1}\right)=H\left(r_{0}\right)+\delta r\left(\frac{d H}{d r}\right)_{r_{0}},
\end{aligned}
$$

de forma que obtemos todas as quantidades em $r_{1}$. Dessa forma, podemos repetir esse mesmo processo para obter essas quantidades em $r_{2}$, e em seguida, em $r_{3}$, e assim por diante. Esse processo é repetido até obtermos os valores das quantidades em $r_{N}=R$, onde $R$ é o raio da estrela. Esse ponto $R$ é definido como o ponto em que a pressão atinge o valor nulo, indicando que atingimos a superfície da estrela

$$
P_{\text {sup }}=0 \text {. }
$$

Assim, para as estrelas de quarks e de hádrons deste trabalho, ao obter o valor de $P\left(r_{i}\right)$, verificamos se esse valor é maior ou menor do que zero. Se for maior, continuamos o procedimento como já explicado; se for menor, definimos $r_{i}=R, M\left(r_{i}\right)=M$, $H\left(r_{i}\right)=H(R)$ e $\beta\left(r_{i}\right)=\beta(R)$, sendo $M$ a massa total da estrela. Com isso, o processo é finalizado. Entretanto, para as estrelas híbridas, por conta da equação que usamos para realizar a transição de fase, podemos notar que o valor da pressão não atinge valores negativos. Dessa forma, definimos a superficíe da estrela de nêutrons como o ponto em que a densidade bariônica se anula. Para os dois tipos de estrelas híbridas aqui utilizadas, a pressão na superficie da estrela será de

$$
P_{\text {sup }} \equiv P\left(\rho_{B}=0\right)=\frac{1}{2}\left(-B+\sqrt{B^{2}+4 \delta_{0}}\right) .
$$

Dessa forma, precisamos verificar, em cada iteração, se $P\left(r_{i}\right)$ é maior ou menor do que $P_{\text {sup. }}$. 
Ao final, teremos o valor de $M, R, H(R)$ e $\beta(R)$ para uma estrela cuja pressão central é de $P\left(r_{0}\right)=P_{c}$. Repetindo o mesmo processo com diferentes valores de $P_{c}$, teremos uma tabela do tipo da tabela B, que nos permite, por exemplo, fazer o diagrama massa-raio, ou obter a deformabilidade de maré e relacioná-la com a massa.

\begin{tabular}{|ccccc|}
\hline$P_{c}\left(\mathrm{MeV} / \mathrm{fm}^{3}\right)$ & $M\left(\mathrm{M}_{\odot}\right)$ & $R(\mathrm{~km})$ & $\mathrm{H}\left(\mathrm{km}^{2}\right)$ & $\beta(\mathrm{km})$ \\
\hline \hline 10 & 9.34 & 1.91 & 48.11 & 12.94 \\
\hline 8.91 & 9.43 & 1.94 & 48.70 & 12.91 \\
\hline 7.94 & 9.53 & 1.97 & 49.45 & 12.89 \\
$\vdots$ & $\vdots$ & $\vdots$ & $\vdots$ & $\vdots$
\end{tabular}

Tabela B.1: Exemplo de resultado obtido ao final de todo o processo de resolução numérica da TOV. 



\section{Deformabilidade de Maré}

Neste apêndice, iremos deduzir as equações de deformabilidade de maré. Para isso, primeiro acharemos o potencial gravitacional newtoniano do sistema binário. Em seguida, usaremos esse potencial na aproximação de campos fracos, no qual nos permitirá deduzir a equação de $k_{2}$ e a equação diferencial que a quantidade $H(r)$ deve satisfazer. Nessa dedução, consideramos as unidades naturais sendo $c=\hbar=G=1$.

\section{C.1 Potencial Gravitacional Newtoniano}

Vamos considerar um sistema binário formado por um corpo A e por um corpo B, este último sendo pontual. Colocamos a origem do sistema no centro de massa do corpo A e queremos saber qual é o potencial gravitacional total sentido por um ponto $\mathrm{P}$ na superfície de A [41]. Esse pontencial pode ser escrito como

$$
\Phi=\Phi_{A}+\Phi_{B}
$$

onde $\Phi_{A}\left(\Phi_{B}\right)$ é a contribuição do potencial gerada pela interação entre o ponto $\mathrm{P}$ e o corpo A (B). Vamos primeiramente obter o pontecial $\Phi_{A}$. Relembrando que o potencial gravitacional no limite newtoniano pode ser escrito como

$$
\Phi_{A}(t, \vec{r})=-\int \frac{\rho\left(t, \vec{r}^{\prime}\right)}{\left|\vec{r}-\vec{r}^{\prime}\right|} d^{3} \vec{r}^{\prime},
$$

podemos fazer uma expansão multipolar no termo $1 /|\vec{r}-\vec{r}|$, obtendo assim

$$
\begin{aligned}
\frac{1}{\left|\vec{r}-\vec{r}^{\prime}\right|} & =\sum_{l=0}^{\infty} \frac{r^{\prime l}}{r^{l+1}} P_{l}\left(\hat{n}^{\prime} \cdot \hat{n}\right) \\
& =\frac{1}{r}+\frac{r^{\prime}}{r^{2}} n_{i}^{\prime} n_{i}+\frac{x^{\prime 2}}{x^{3}} \frac{1}{2}\left[3\left(n_{i}^{\prime} n_{i}\right)^{2}-1\right]+\mathcal{O}\left(r^{-4}\right) \\
& =\frac{1}{r}+\frac{r^{\prime}}{r^{2}} \frac{r_{i}^{\prime}}{r^{\prime}} n_{i}+\frac{3}{2 r^{3}}\left[r_{i}^{\prime} r_{j}^{\prime} n_{i} n_{j}-\frac{1}{3} r^{\prime 2}\right]+\mathcal{O}\left(r^{-4}\right),
\end{aligned}
$$


onde $\vec{r}$ é a posição do ponto $\mathrm{P}, \vec{r}^{\prime}$ a posição da contribuição de uma massa infinitesimal do corpo A e o quadrado do módulo e o versor do vetor $\vec{r}$ são dados, respectimente, por $r^{2}=r_{i} r_{j} \delta_{i j}$ e $n_{i}=r_{i} / r$ (e, analogamente, para $\vec{r}^{\prime}$ ). Como $\hat{n}$ é o versor do vetor $\vec{r}$, por definição ele satisfaz $|\vec{n}|=1$. Dessa forma, multiplicando o segundo termo entre colchetes por $n^{2}=n_{i} n_{j} \delta_{i j}(=1)$, temos

$$
\begin{aligned}
\frac{1}{\left|\vec{r}-\vec{r}^{\prime}\right|} & =\frac{1}{r}+\frac{r_{i}^{\prime}}{r^{2}} n_{i}+\frac{3}{2 r^{3}}\left[r_{i}^{\prime} r_{j}^{\prime} n_{i} n_{j}-\frac{1}{3} r^{\prime 2} n^{2}\right]+\mathcal{O}\left(r^{-4}\right) \\
& =\frac{1}{r}+\frac{r_{i}^{\prime}}{r^{2}} n_{i}+\frac{3}{2 r^{3}}\left[r_{i}^{\prime} r_{j}^{\prime}-\frac{1}{3} r^{\prime 2} \delta_{i j}\right] n_{i} n_{j}+\mathcal{O}\left(r^{-4}\right) .
\end{aligned}
$$

Substituindo esse resultado na equação (C.1.2), obtemos

$$
\begin{aligned}
\Phi_{A}(t, \vec{r})= & -\frac{1}{r} \int \rho_{A}\left(t, \vec{r}^{\prime}\right) d^{3} \vec{r}^{\prime}-\frac{n_{i}}{r^{2}} \int r_{i}^{\prime} \rho_{A}\left(t, \vec{r}^{\prime}\right) d^{3} \vec{r}^{\prime} \\
& -\frac{3}{2 r^{3}} n_{i} n_{j} \int\left[r_{i}^{\prime} r_{j}^{\prime}-\frac{1}{3} r^{\prime 2} \delta_{i j}\right] \rho_{A}\left(t, \vec{r}^{\prime}\right) d^{3} \vec{r}^{\prime}+\mathcal{O}\left(r^{-4}\right) .
\end{aligned}
$$

A integral do primeiro termo é a massa total do corpo A, resultando no potencial newtoniano de um corpo pontual (o que era esperado, já que esse é o termo de monopolo). Já a integral do segundo termo se anula [66]. Com isso, podemos escrever o potencial gravitacional gerado pelo corpo A como

$$
\Phi_{A}=-\frac{M}{r}-\frac{3}{2 r^{3}} Q_{i j} n_{i} n_{j}+\mathcal{O}\left(r^{-4}\right)
$$

onde

$$
Q_{i j}=\int\left[r_{i}^{\prime} r_{j}^{\prime}-\frac{1}{3} r^{\prime 2} \delta_{i j}\right] \rho_{A}\left(t, \vec{r}^{\prime}\right) d^{3} \vec{r}^{\prime}
$$

é o momento de quadrupolo. Aqui, podemos ressaltar que esse tensor $Q_{i j}$ é um tensor simétrico cujo traço é nulo. Por conta disso, podemos escrevê-lo em termos dos harmônicos esféricos [41, 66, 40]

$$
Q_{i j}=\sum_{m=-2}^{2} Q_{m} \mathcal{Y}_{i j}^{2 m},
$$

sendo $\mathcal{Y}_{i j}^{2 m}$ o tensor simétrico de traço nulo definido por

$$
Y_{2 m}(\theta, \phi)=\mathcal{Y}_{i j}^{2 m} n_{i} n_{j}
$$

onde $Y_{2 m}(\theta, \phi)$ são os harmônicos esféricos de $l=2$ e $\vec{n}=(\sin \theta \cos \phi, \sin \theta \sin \phi, \cos \theta)$.

Agora vamos calcular o potencial gravitacional gerado pela interação do corpo B sobre o ponto P. Claramente, ele também é dado pela equação

$$
\Phi_{B}(t, \vec{r})=-\int \frac{\rho_{B}\left(t, \vec{r}^{\prime \prime}\right)}{\left|\vec{r}-\vec{r}^{\prime \prime}\right|} d^{3} \vec{r}^{\prime \prime},
$$


onde $\vec{r}$ é a posição do ponto $\mathrm{P}$ e $\vec{r}^{\prime \prime}$, a posição do corpo B. Aqui a ideia também é fazer uma expansão; porém, por conta do corpo $\mathrm{B}$ estar distante do ponto $\mathrm{P}$, podemos dizer que o ponto $\mathrm{P}$ está aproximadamente na origem. Em outras palavras, faremos uma expansão em série de Taylor em torno de $\vec{r}=0$. Dessa forma, teremos

$$
\Phi_{B}=\Phi_{B}(0)+\left.\partial_{i} \Phi_{B}\right|_{0} r^{i}+\left.\frac{1}{2} \partial_{i j}^{2} \Phi_{B}\right|_{0} r^{i} r^{j}+\mathcal{O}\left(r^{3}\right) .
$$

Podemos definir que o valor do potencial $\Phi_{B}$ é nulo na origem, de forma que o primeiro termo da expansão se torne zero. O segundo termo da expansão é a aceleração do centro de massa da fonte, em que podemos assumir que este é estático [66]. Com isso, o potencial pode ser escrito como

$$
\Phi_{B}=-\varepsilon_{i j} r^{i} r^{j}+\mathcal{O}\left(r^{3}\right)
$$

onde

$$
\varepsilon_{i j}=-\left.\frac{1}{2} \partial_{i j}^{2} \Phi_{B}\right|_{0}
$$

é o termo de quadrupolo do campo de deformabilidade. Assim como $Q_{i j}$, $\varepsilon_{i j}$ também é um tensor simétrico de traço nulo. Notemos que, como o potencial gravitacional deve satisfazer a equação de Laplace, temos que, no vácuo,

$$
\nabla^{2} \Phi_{B}=0 \Rightarrow-\varepsilon_{11}-\varepsilon_{22}-\varepsilon_{33}=0 \Rightarrow \operatorname{Tr}(\varepsilon)=0
$$

Com isso, analogamente ao caso do tensor $Q_{i j}$, podemos escrever $\varepsilon_{i j}$ como

$$
\varepsilon_{i j}=\sum_{m=-2}^{2} \varepsilon_{m} \mathcal{Y}_{i j}^{2 m} .
$$

Agora que temos o potencial gravitacional gerado pelo corpo A e pelo corpo B, podemos ver que o potencial total no ponto $\mathrm{P}$ é de

$$
\Phi=-\frac{M}{r}-\frac{3}{2 r^{3}} Q_{i j} n_{i} n_{j}+\mathcal{O}\left(r^{-4}\right)+\varepsilon_{i j} r^{i} r^{j}+\mathcal{O}\left(r^{3}\right) .
$$

\section{C.2 Tidal Love Number $k_{2}$}

Nesta seção, queremos deduzir qual a relação entre o quanto o corpo A é deformado (que está relacionado com a quantidade $Q_{i j}$ ) devido ao campo de deformabilidade causado pelo corpo B (relacionado com $\varepsilon_{i j}$ ). Na aproximação de campos fracos, podemos escrever que [67, 68]

$$
\Phi=\frac{1}{2}\left(g_{00}-1\right)=-\frac{M}{r}-\frac{3}{2 r^{3}} Q_{i j} n_{i} n_{j}+\mathcal{O}\left(r^{-4}\right)+\frac{1}{2} r^{2} \varepsilon_{i j} n_{i} n_{j}+\mathcal{O}\left(r^{3}\right),
$$


onde usamos a equação (C.1.10) na segunda igualdade. Apesar da equação (C.1.10) continuar válida, o mesmo não acontece para os tensores $Q_{i j}$ e $\varepsilon_{i j}$. Eles continuam sendo tensores simétricos e de traço nulo (e portanto, as equações (C.1.5) e (C.1.9) podem continuar sendo usadas nesta seção); porém, as equações (C.1.4) e (C.1.8) não são válidas para o caso relativístico [40]. Por conta disso, iremos então considerar que a relação entre $Q_{i j}$ e $\varepsilon_{i j}$ pode ser aproximada por uma equação linear, como

$$
Q_{m}=-\lambda \varepsilon_{m},
$$

de forma que a quantidade $\lambda$ nos fornece uma medida da resposta do momento multipolar em razão ao campo de deformabilidade externa [66]. Por convenção, escrevermos

$$
\lambda=\frac{2}{3} k_{2} R^{5}
$$

de forma que $k_{2}$ é a quantidade adimensional que nos informa sobre o quanto um corpo deforma devido a um campo de deformabilidade. Para obtermos a equação de $k_{2}$, o primeiro passo é encontrar o valor de $g_{00}$ para podermos substituir na equação (C.2.1). Para isso, vamos discutir o que acontece com a métrica em um sistema binário. No sistema binário de corpos compactos, o segundo corpo irá causar uma perturbação na métrica do espaço-tempo do primeiro. Dessa forma, considerando uma perturbação linear, podemos dizer que a métrica do espaço-tempo de um corpo do sistema pode ser escrita como

$$
g_{\mu \nu}=g_{\mu \nu}^{(0)}+h_{\mu \nu}
$$

onde $g_{\mu \nu}^{(0)}$ é a métrica de Schwarzschild e $h_{\mu \nu}$ é a perturbação (e portanto, $\left|h_{\mu \nu}\right|<<1$ ). Com isso, para descobrirmos o valor de $g_{00}$, precisamos encontrar $h_{00}$. Em [40], é analizada a dependência angular de $h_{\mu \nu}$ em harmônicos esféricos e considerada perturbações estáticas, de $l=2$ e de paridade par no gauge de Regge-Wheeler [69]. Feito isso, é possível obter que $[40,70]$

$$
h_{\mu \nu}=Y_{2 m}(\theta, \phi) \operatorname{diag}\left[e^{2 \nu(r)} H_{0}(r),-e^{2 \lambda(r)} H_{2}(r),-r^{2} K(r),-r^{2} \sin ^{2} \theta K(r)\right] \text {, }
$$

onde $H_{0}(r), H_{2}(r)$ e $K(r)$ são funções a serem descobertas. Com a perturbação da métrica escrita dessa forma, podemos obter a variação dos tensores de Einstein $\delta G_{\mu}^{\nu}$ e, juntamente com a equação de Einstein linearizada

$$
\delta G_{\mu}^{\nu}=8 \pi \delta T_{\mu}^{\nu}
$$

sendo

$$
\delta T_{\mu}^{\nu}=\operatorname{diag}(\delta \epsilon,-\delta P,-\delta P,-\delta P)
$$


a variação do tensor momento energia, é possível obter, após alguns cálculos, que a função $H_{0}(r)=H_{2}(r) \equiv H(r)$ deve satisfazer a equação [40, 71, 72]

$$
\begin{aligned}
& H^{\prime \prime}(r)+H^{\prime}(r)\left\{\frac{2}{r}+e^{2 \lambda}\left[\frac{2 M(r)}{r^{2}}+4 \pi r(P(r)-\epsilon(r))\right]\right\} \\
& \quad+H(r)\left[-\frac{6 e^{2 \lambda}}{r^{2}}+4 \pi e^{2 \lambda(r)}\left(5 \epsilon(r)+9 P(r)+\frac{\epsilon(r)+P(r)}{d P / d \epsilon}\right)-\left(2 \nu^{\prime}\right)^{2}\right]=0 .
\end{aligned}
$$

Como nosso objetivo aqui é achar $g_{00}$ para substituir na equação (C.2.1), precisamos encontrar a solução de $H(r)$ fora da estrela; ou seja, no vácuo. Dessa forma, o tensor de momento-energia se torna nulo (e portanto, $P(r)=\epsilon(r)=0$ ), e a equação da função $H(r)$ se torna

$$
H^{\prime \prime}(r)+H^{\prime}(r)\left[\frac{2}{r}+e^{2 \lambda} \frac{2 M}{r^{2}}\right]+H(r)\left[-\frac{6 e^{2 \lambda}}{r^{2}}-\left(2 \nu^{\prime}\right)^{2}\right]=0 .
$$

Usando os resultados obtidos no apêndice A,

$$
\begin{aligned}
e^{2 \lambda} & =\left(1-\frac{2 M}{r}\right)^{-1}, \\
\nu^{\prime}(r) & =-\frac{1}{2 r}\left[1-\left(1+8 \pi r^{2} P(r)\right) e^{2 \lambda}\right] \\
& =-\frac{1}{2 r}\left(1-e^{2 \lambda}\right) \\
& =\frac{M}{r^{2}-2 r M},
\end{aligned}
$$

onde tomamos $P=0$ na segunda igualdade de $\nu^{\prime}(r)$, substituimos esses resultados obtidos na equação (C.2.9), resultando em

$$
H^{\prime \prime}(r)+H^{\prime}(r)\left[\frac{2}{r}+\frac{2 M}{r^{2}-2 M}\right]-H(r)\left[\frac{6}{r^{2}} \frac{1}{1-2 M / r}+\frac{4 M^{2}}{\left(r^{2}-2 r M\right)^{2}}\right]=0 .
$$

Fazendo a mudança de variável

$$
x=\frac{r}{M}-1,
$$

podemos obter que

$$
H^{\prime \prime}(x) \frac{1}{M^{2}}+H^{\prime}(x) \frac{1}{M} \frac{2 x}{M\left(x^{2}-1\right)}-H(x) \frac{6 x^{2}-2}{M^{2}\left(x^{2}-1\right)^{2}}=0 .
$$

Multiplicando ambos os lados por $M^{2}\left(x^{2}-1\right)$,

$$
\left(x^{2}-1\right) H^{\prime \prime}(x)+2 x H^{\prime}(x)-H(x) \frac{6 x^{2}-2}{\left(x^{2}-1\right)}=0,
$$


e somando e subtraindo por 6 no numerador do termo proporcional a $H(x)$, essa equação fica sendo

$$
\left(x^{2}-1\right) H^{\prime \prime}(x)+2 x H^{\prime}(x)-H(x)\left(6+\frac{4}{x^{2}-1}\right)=0 .
$$

Essa equação de $H(x)$ toma a mesma forma da equação de Legendre associada para $l=m=2$. Dessa forma, sabemos então que a sua solução deve ser do tipo

$$
H(x)=c_{1} Q_{2}^{2}(x)+c_{2} P_{2}^{2}(x)
$$

onde $P_{l}^{m}(x)$ e $Q_{l}^{m}(x)$ são as funções de Legendre associadas, dadas por

$$
\begin{gathered}
P_{l}^{m}(x)=\frac{(-1)^{m}}{2^{l} l !}\left(1-x^{2}\right)^{m / 2} \frac{d^{l+m}}{d x^{l+m}}\left(x^{2}-1\right)^{l}, \\
Q_{l}^{m}(x)=\frac{\sqrt{\pi} \Gamma(l+m+1)}{2^{l+1} \Gamma(l+3 / 2)} \frac{1}{x^{l+m+1}}\left(1-x^{2}\right)^{m / 2} \\
\times{ }_{2} F_{1}\left(\frac{l+m+1}{2}, \frac{l+m+2}{2}, l+\frac{3}{2}, \frac{1}{x^{2}}\right),
\end{gathered}
$$

$\mathrm{e}_{2} F_{1}(\alpha, \beta, \gamma, x)$ a função hipergeométrica dada por

$$
{ }_{2} F_{1}(\alpha, \beta, \gamma, x)=\frac{\Gamma(\gamma)}{\Gamma(\alpha) \Gamma(\beta)} \sum_{n=0}^{\infty} \frac{\Gamma(n+\alpha) \Gamma(n+\beta)}{\Gamma(n+\gamma) n !} x^{n} .
$$

Para o caso $l=m=2$, essas equações se tornam

$$
\begin{aligned}
P_{2}^{2}(x) & =3\left(1-x^{2}\right) \\
Q_{2}^{2}(x) & =\frac{x\left(3 x^{2}-5\right)}{x^{2}-1}-\frac{3}{2}\left(x^{2}-1\right) \ln \left(\frac{1+1 / x}{1-1 / x}\right) .
\end{aligned}
$$

Substituindo $x=r / M-1$, obtemos que

$$
\begin{aligned}
P_{2}^{2}(r)= & -3\left(\frac{r}{M}\right)^{2}\left(1-\frac{2 M}{r}\right), \\
Q_{2}^{2}(r)= & -\left(\frac{r}{M}\right)^{2}\left(1-\frac{2 M}{r}\right) \\
& \times\left[-\frac{M(M-r)\left(2 M^{2}+6 M r-3 r^{2}\right)}{r^{2}(r-2 M)^{2}}+\frac{3}{2} \ln \left(\frac{r}{r-2 M}\right)\right] .
\end{aligned}
$$

Tomando valores grandes de $r$ ou, em outras palavras, definindo $a=1 / r$ e expandindo em série de Taylor em torno de $a=0$, obtemos

$$
\begin{aligned}
P_{2}^{2}(r) & =3\left(\frac{r}{M}\right)^{2}+(\mathcal{O}(1 / r))^{-1}, \\
Q_{2}^{2}(r) & =\frac{8}{5}\left(\frac{M}{r}\right)^{3}+\mathcal{O}\left(1 / r^{4}\right) .
\end{aligned}
$$


Dessa forma, pegando apenas o primeiro termo de cada função, podemos escreve a função $H(r)$ como

$$
H(r)=\frac{8}{5}\left(\frac{M}{r}\right)^{3} c_{1}+3\left(\frac{r}{M}\right)^{2} c_{2} .
$$

Tendo essa expressão da função $H(r)$, podemos obter que a métrica do espaço pode ser escrita como

$$
\begin{aligned}
g_{00} & =g_{00}^{(0)}+h_{00} \\
& =e^{2 \nu(r)}\left[1+H(r) Y_{2 m}(\theta, \phi)\right] \\
& =\left(1-\frac{2 M}{r}\right)\left\{1+\left[\frac{8}{5}\left(\frac{M}{r}\right)^{3} c_{1}+3\left(\frac{r}{M}\right)^{2} c_{2}\right] Y_{2 m}(\theta, \phi)\right\},
\end{aligned}
$$

onde usamos que (dedução no apêndice A)

$$
e^{2 \nu(r)}=1-\frac{2 M}{r}
$$

Substituindo na equação (C.2.1) e igualando os termos de mesma ordem em $r$, vemos que o termo proporcional a $1 / r^{3}$ nos fornece

$$
\frac{1}{2} \frac{8}{5}\left(\frac{M}{r}\right)^{3} c_{1} Y_{2 m}(\theta, \phi)=-\frac{3}{2 r^{3}} Q_{i j} n_{i} n_{j},
$$

e usando as equações (C.1.5) e (C.2.2) podemos obter que

$$
\frac{1}{2} \frac{8}{5}\left(\frac{M}{r}\right)^{3} c_{1} Y_{2 m}(\theta, \phi)=+\frac{3}{2 r^{3}} \lambda \varepsilon_{m} Y_{2 m}(\theta, \phi) .
$$

Isolando $c_{1}$, obtemos que

$$
c_{1}=\frac{15}{8} \frac{1}{M^{3}} \lambda \varepsilon
$$

Já o termo proporcional a $r^{2}$, resulta em

$$
\frac{1}{2} 3\left(\frac{r}{M}\right)^{2} c_{2} Y_{2 m}(\theta, \phi)=\frac{1}{2} r^{2} \varepsilon_{i j} n_{i} n_{j} .
$$

Usando a equação (C.1.9) e isolando $c_{2}$, obtemos

$$
c_{2}=\frac{1}{3} M^{2} \varepsilon
$$

Substituindo $c_{1}$ e $c_{2}$ obtidos agora e as equações (C.2.22) e (C.2.23) na equação (C.2.16), obtemos uma expressão de $H(r)$ que depende das variáveis $\varepsilon$ e $\lambda$, dada por

$$
\begin{aligned}
H(r)= & \frac{1}{8 M^{5}}\left(1-\frac{2 M}{r}\right) r^{2} \varepsilon\left\{8 M^{5}\right. \\
& \left.+15 \lambda\left[-\frac{M(M-r)\left(2 M^{2}+6 M r-3 r^{2}\right)}{r^{2}(r-2 M)^{2}}+\frac{3}{2} \ln \left(\frac{r}{r-2 M}\right)\right]\right\} .
\end{aligned}
$$


Derivando em relação a $r$,

$$
\begin{aligned}
H^{\prime}(r)= & \frac{1}{8} \varepsilon\left[16(r-M)-\frac{30\left(2 M^{4}-2 M^{3} r+13 M^{2} r^{2}-12 M r^{3}+3 r^{4}\right) \lambda}{M^{4} r^{2}(r-2 M)^{2}}\right. \\
& \left.+\frac{45(r-M)}{M^{5}} \lambda \ln \left(\frac{r}{r-2 M}\right)\right] .
\end{aligned}
$$

Supondo que conhecemos os valores de $H(r)$ e $H^{\prime}(r)$ em $r=R$, essas últimas equações formam um sistema de duas equações com duas incógnitas ( $\varepsilon$ e $\lambda$ ); ou seja, possível de ser resolvido. Com isso, tomando $r=R$, da equação (C.2.28), isolamos a variável $\varepsilon$ e substituímos na equação (C.2.27), resultando em uma equação que depende apenas de $\lambda$. Isolando $\lambda \mathrm{e}$, para simplificar a expressão, definimos

$$
H^{\prime}(R)=\frac{y}{R} H(R), \quad M=R C,
$$

obtemos

$$
\begin{aligned}
\lambda= & 16(1-2 C)^{2} C^{5} R^{5}[2+2 C(y-1)-y]\{30 C[6-3 y+C(3\{5 y-8\} \\
& +2 C\{13-11 y+C[-2+3 y+2 C(1+y)]\})] \\
& \left.-45(1-2 C)^{2}(2+2 C\{y-1\}-y) \ln \left(\frac{1}{1-2 C}\right)\right\}^{-1} .
\end{aligned}
$$

Por fim, usando a expressão (C.2.3) e isolando $k_{2}$, obtemos

$$
\begin{aligned}
k_{2}= & \frac{8 C^{5}}{5}(1-2 C)^{2}[2+2 C(y-1)-y]\{2 C[6-3 y+3 C(5 y-8)] \\
& +4 C^{3}\left[13-11 y+C(3 y-2)+2 C^{2}(1+y)\right] \\
& \left.+3(1-2 C)^{2}[2-y+2 C(y-1)] \ln (1-2 C)\right\}^{-1},
\end{aligned}
$$

que, da equação (C.2.29), temos

$$
y=\frac{R H^{\prime}(R)}{H(R)}, \quad C=\frac{M}{R}
$$

onde $C$ é a compacidade da estrela. Dessa forma, sabendo os valores de $H(R)$ e $H^{\prime}(R)$, podemos obter o valor de $k_{2}$ a partir da equação deduzida.

Para obtermos os valores de $H(R)$ e $H^{\prime}(R)$, podemos resolver a equação (C.2.8) numericamente no intervalo $0 \leq r \leq R$. Para isso, podemos reescrever essa equação diferencial de segunda ordem em um sistema de duas equações diferenciais de primeira 
ordem, como

$$
\begin{aligned}
\frac{d H}{d r}= & \beta(r) \\
\frac{d \beta}{d r}= & 2\left(1-\frac{2 M(r)}{r}\right)^{-1} H(r)\left\{-2 \pi\left[5 \epsilon(r)+9 P(r)+\frac{\epsilon(r)+P(r)}{d P / d \epsilon}\right]\right. \\
& \left.+\frac{3}{r^{2}}+2\left(1-\frac{2 M(r)}{r}\right)^{-1}\left(\frac{M(r)}{r^{2}}+4 \pi r P(r)\right)^{2}\right\} \\
& +\frac{2 \beta(r)}{r}\left(1-\frac{2 M(r)}{r}\right)^{-1}\left[-1+\frac{M(r)}{r}+2 \pi r^{2}(\epsilon(r)-P(r))\right],
\end{aligned}
$$

e assim, sabendo as condições de contorno, podemos resolver esse sistema juntamente com as equações de TOV. Esse processo numérico é melhor explicado no apêndice B. Sendo assim, ainda nos falta a informação das condições de contorno, dadas pelos valores de $H(0)$ e $\beta(0)$. Para isso, voltamos novamente para a equação (C.2.8) e, assumindo que $H(r)$ é regular em $r=0$ e resolvendo a equação para $r$ próximo de zero, podemos obter que $[40,41]$

$$
H(r)=a_{0} r^{2}\left[1-\frac{2 \pi}{7}\left(5 \epsilon(0)+9 P(0)+\frac{\epsilon(0)+P(0)}{d P /\left.d \epsilon\right|_{0}}\right) r^{2}+\prime\left(r^{3}\right)\right] .
$$

Tomando apenas o primeiro termo e derivando para obter a condição de contorno de $\beta$, temos

$$
H(r=0) \approx a_{0} r^{2}, \quad \beta(r=0) \approx 2 a_{0} r .
$$

Como nosso objetivo é obter o valor de $y$, então o que realmente nos interessa é a quantidade $\beta(R) / H(R)$. Dessa forma, podemos tomar o valor de $a_{0}=1$.

Com isso, concluímos que, para obter o valor de $\Lambda$, resolvemos numericamente o sistema de equações diferenciais (C.2.32) e (C.2.33) usando as condições de contorno (C.2.35) pelo método explicado no apêndice B. Em seguida, obtemos o valor de $y$ pela equação

$$
y=\frac{R \beta(R)}{H(R)}-\frac{4 \pi R^{3} \epsilon_{\text {sup }}}{M},
$$

onde o termo adicional em relação à equação de $y$ dedudiza (equação (C.2.31)) é uma correção para o caso da densidade de energia superficial $\epsilon_{\text {sup }} \equiv \epsilon(P=0)$ não ser nula [39]. Substituindo o valor de $y$ na equação (C.2.30), obtemos o valor de $k_{2}$. Por fim, substituindo $k_{2}$ na equação (2.2.1), obtemos o valor de $\Lambda$ e podemos comparar com os dados experimentais. 



\section{Revisão da QCD}

A lagrangiana da QCD é dada por

$$
\mathcal{L}_{\mathrm{QCD}}=-\frac{1}{4} F_{\mu \nu}^{a} F^{a \mu \nu}+\sum_{q=1}^{N_{f}} \bar{\psi}_{i}^{q}\left[i \gamma^{\mu}\left(\delta_{i j} \partial_{\mu}-i g T_{i j}^{a} G_{\mu}^{a}\right)-\delta_{i j} m_{q}\right] \psi_{j}^{q},
$$

sendo $q$ o sabor dos quarks ( $q=1,2,3$, referente aos quarks up, down e strange); $i$ e $j$, a cor do quark ( $i, j=1,2,3$, referente às cores red, green e blue); $a$, a cor do glúon $(a=1, \ldots, 8) ; g$, a constante de acoplamento do SU(3);

$$
F^{a \mu \nu}=\partial^{\mu} G^{a \nu}-\partial^{\nu} G^{a \mu}+g f^{a b c} G^{b \mu} G^{c \nu},
$$

onde $G_{\mu}^{a}$ é o campo dos glúons e $\psi$, o campo dos quarks e $f^{a b c}$, a constante de acoplamento do SU(3), que satisfaz a propriedade

$$
f_{a c d} f_{b c d}=3 \delta_{a b} .
$$

$T^{a}$ são os geradores do grupo $\mathrm{SU}(3)$, dados por

$$
T^{a}=\frac{\lambda^{a}}{2}, \quad a=1, \ldots 8,
$$

e $\lambda^{a}$ são as matrizes de Gerll-Mann

$$
\begin{gathered}
\lambda^{1}=\left[\begin{array}{lll}
0 & 1 & 0 \\
1 & 0 & 0 \\
0 & 0 & 0
\end{array}\right], \quad \lambda^{2}=\left[\begin{array}{ccc}
0 & -i & 0 \\
i & 0 & 0 \\
0 & 0 & 0
\end{array}\right], \quad \lambda^{3}=\left[\begin{array}{ccc}
1 & 0 & 0 \\
0 & -1 & 0 \\
0 & 0 & 0
\end{array}\right], \\
\lambda^{4}=\left[\begin{array}{lll}
0 & 0 & 1 \\
0 & 0 & 0 \\
1 & 0 & 0
\end{array}\right], \quad \lambda^{5}=\left[\begin{array}{ccc}
0 & 0 & -i \\
0 & 0 & 0 \\
i & 0 & 0
\end{array}\right], \quad \lambda^{6}=\left[\begin{array}{lll}
0 & 0 & 0 \\
0 & 0 & 1 \\
0 & 1 & 0
\end{array}\right], \\
\lambda^{7}=\left[\begin{array}{ccc}
0 & 0 & 0 \\
0 & 0 & -i \\
0 & i & 0
\end{array}\right], \quad \lambda^{8}=\frac{1}{\sqrt{3}}\left[\begin{array}{ccc}
1 & 0 & 0 \\
0 & 1 & 0 \\
0 & 0 & -2
\end{array}\right] .
\end{gathered}
$$




\section{D.1 Equação (3.2.39)}

Para um único sabor de quark, a densidade de quarks (equação (3.2.39)) aplicada em um sistema de $\mathrm{N}$ quarks pode ser escrita como

$$
\rho=\left\langle N\left|\psi_{i}^{\dagger} \psi_{i}\right| N\right\rangle,
$$

onde $\psi_{i}$ é o campo dos quarks e estamos mantendo implícito o índice $q$. No caso do espectro de energia positiva, podemos escrevê-lo como [51]

$$
\psi_{i}=c_{i} \psi=c_{i} \frac{1}{\sqrt{V}} \sum_{\vec{k}, \lambda} A_{\vec{k}}^{(\lambda)} u^{(\lambda)}(\vec{k}) e^{-i k \cdot x},
$$

sendo $\vec{k}$ e $\lambda$ o momento e o spin do quark; $u^{(\lambda)}(\vec{k})$, um spinor de Dirac, que deve satisfazer a relação

$$
\left(u^{\left(\lambda^{\prime}\right)}\left(\vec{k}^{\prime}\right)\right)^{\dagger} u^{(\lambda)}(\vec{k})=\delta_{\vec{k}, \vec{k}^{\prime}} \delta_{\lambda, \lambda^{\prime}}
$$

$A_{\vec{k}}^{(\lambda)}$, o operador de aniquilação, que deve respeitar a relação de anticomutação

$$
\left\{A_{\vec{k}}^{(\lambda)},\left(A_{\vec{k}}^{(\lambda)}\right)^{\dagger}\right\}=1
$$

$k \cdot x=k_{\mu} x^{\mu}$ e $c_{i}$ é a carga de cor, sendo elas

$$
c_{1}=\left[\begin{array}{l}
1 \\
0 \\
0
\end{array}\right] \text {, para vermelho, } c_{2}=\left[\begin{array}{l}
0 \\
1 \\
0
\end{array}\right] \text {, para azul, } c_{3}=\left[\begin{array}{l}
0 \\
0 \\
1
\end{array}\right] \text {, para verde }
$$

Da equação (D.1.2), podemos obter

$$
\psi_{i}^{\dagger}=\psi^{\dagger} c_{i}^{\dagger}=c_{i}^{\dagger} \frac{1}{\sqrt{V}} \sum_{\vec{k}, \lambda}\left(A_{\vec{k}}^{(\lambda)}\right)^{\dagger}\left(u^{(\lambda)}(\vec{k})\right)^{\dagger} e^{i k \cdot x} .
$$

Com isso, substituindo as equações (D.1.6) e (D.1.2) na equação (D.1.1), obtemos

$$
\begin{aligned}
\rho & =\left\langle N\left|\psi_{i}^{\dagger} \psi_{i}\right| N\right\rangle \\
& =c_{i}^{\dagger} c_{i} \frac{1}{V} \sum_{\vec{k}, \vec{k}^{\prime}, \lambda, \lambda^{\prime}}\left(u^{\left(\lambda^{\prime}\right)}\left(\vec{k}^{\prime}\right)\right)^{\dagger} u^{(\lambda)}(\vec{k}) e^{i k^{\prime} \cdot x} e^{-i k \cdot x}\left\langle N\left|\left[A_{\vec{k}^{\prime}}^{\left(\lambda^{\prime} \dagger^{\dagger}\right.} A_{\vec{k}}^{(\lambda)}\right]\right| N\right\rangle \\
& =\frac{3}{V} \sum_{\vec{k}, \lambda}\left\langle N\left|\left[A_{\vec{k}}^{(\lambda)^{\dagger}} A_{\vec{k}}^{(\lambda)}\right]\right| N\right\rangle,
\end{aligned}
$$

onde usamos a propriedade dada na equação (D.1.3) e as equações (D.1.5) para calcular $c_{i}^{\dagger} c_{i}=c_{1}^{\dagger} c_{1}+c_{2}^{\dagger} c_{2}+c_{3}^{\dagger} c_{3}=3$. Notando que $\left\langle N\left|\left[A_{\vec{k}}^{(\lambda)^{\dagger}} A_{\vec{k}}^{(\lambda)}\right]\right| N\right\rangle=1$, obtemos

$$
\rho=\frac{3}{V} \sum_{\vec{k}, \lambda} 1 \text {. }
$$


Fazendo a troca de momentos discretos para contínuos, dada por

$$
\frac{1}{V} \sum_{\vec{k}, \lambda} \rightarrow \frac{\gamma_{Q}}{\left(2 \pi^{3}\right)} \int d^{3} k
$$

onde $\gamma_{Q}=2($ spin $) \times 3$ (cor) é o fator de degenerescência dos quarks, a densidade de quarks fica sendo

$$
\begin{aligned}
\rho & =3 \frac{\gamma_{Q}}{\left(2 \pi^{3}\right)} \int d^{3} k \\
& =3 \frac{\gamma_{Q}}{2 \pi^{2}} \int_{0}^{k_{F}} d k k^{2} .
\end{aligned}
$$

Calculando a integral, obtemos

$$
\rho^{q}=\frac{\gamma_{Q}}{2 \pi^{2}} k_{F, q}^{3},
$$

em que voltamos a indicar o sabor do quark $q$. Somando sobre os sabores dos quarks, obtemos

$$
\rho=\frac{\gamma_{Q}}{2 \pi^{2}} \sum_{q=1}^{N_{f}} k_{F, q}^{3}
$$

que é a equação (3.2.39).

\section{D.2 Equação (3.2.37)}

Partimos da equação de movimento dos quarks (equação (3.2.26))

$$
\left(i \gamma^{\mu} \partial_{\mu}+g \gamma^{0} T^{a} \alpha_{0}^{a}-m\right) \psi=0
$$

onde deixamos implícito o índice de sabor dos quarks $q$. Multiplicando $\bar{\psi}=\psi^{\dagger} \gamma^{0}$ pela esquerda, obtemos

$$
\bar{\psi}_{i}\left(i \delta_{i j} \gamma^{\mu} \partial_{\mu}+g \gamma^{0} T_{i j}^{a} \alpha_{0}^{a}-m \delta_{i j}\right) \psi_{j}=0 .
$$

Substituindo as equações (D.1.2) e (D.1.6) nesta última, temos que

$$
\frac{1}{V} \sum_{\vec{k}, \vec{k}^{\prime}, \lambda, \lambda^{\prime}} u^{\prime \dagger} A^{\prime \dagger} e^{i k^{\prime} \cdot x} c_{i}^{\dagger}\left(i \delta_{i j} \partial_{0}+i \delta_{i j} \vec{\alpha} \cdot \nabla+g T_{i j}^{a} \alpha_{0}^{a}-m \gamma^{0} \delta_{i j}\right) c_{j} A u e^{-i k \cdot x}=0,
$$

onde usamos que $\gamma^{0} \gamma^{0}=1, \gamma^{0} \vec{\gamma}=\vec{\alpha}$ e simplificamos a notação para $A_{\vec{k}}^{(\lambda)} \equiv A, A_{\vec{k}^{\prime}}^{\left(\lambda^{\prime}\right)} \equiv$ $A^{\prime}, u^{(\lambda)}(\vec{k}) \equiv u$ e $u^{\left(\lambda^{\prime}\right)}\left(\vec{k}^{\prime}\right) \equiv u^{\prime}$. Aplicando as derivadas e rearranjando os termos, obtemos que

$$
\begin{aligned}
\frac{1}{V} \sum_{\vec{k}, \vec{k}^{\prime}, \lambda, \lambda^{\prime}} & \left(i\left(c_{i}^{\dagger} \delta_{i j} c_{j}\right)(-i E)+i\left(c_{i}^{\dagger} \delta_{i j} c_{j}\right) \vec{\alpha} \cdot(i \vec{k})\right. \\
& \left.\quad+g\left(c_{i}^{\dagger} T_{i j}^{a} c_{j} \alpha_{0}^{a}\right)-m \gamma^{0}\left(c_{i}^{\dagger} \delta_{i j} c_{j}\right)\right) A^{\prime \dagger} A u^{\prime \dagger} u e^{-i\left(k-k^{\prime}\right) \cdot x}=0 .
\end{aligned}
$$


Das equações (D.1.5), obtemos que $c_{i}^{\dagger} \delta_{i j} c_{j}=c_{i}^{\dagger} c_{i}=3$; e juntamente com os geradores do grupo SU(3), obtemos

$$
c_{i}^{\dagger} T_{i j}^{a} c_{j} \alpha_{0}^{a}=\alpha_{0}^{1}+\alpha_{0}^{4}+\alpha_{0}^{6} \equiv \mathcal{A},
$$

onde a última igualdade é uma definição para facilitar a notação. Usando esses resultados e a propriedade dada pela equação (D.1.3), ficamos com

$$
\frac{1}{V} \sum_{\vec{k}, \lambda}\left(3 E-3 \vec{\alpha} \cdot \vec{k}+g \mathcal{A}-3 m \gamma^{0}\right) A^{\prime \dagger} A=0
$$

o que implica em

$$
3 E-3 \vec{\alpha} \cdot \vec{k}+g \mathcal{A}-3 m \gamma^{0}=0
$$

Reorganizando os termos

$$
3 E+g \mathcal{A}=3\left(\vec{\alpha} \cdot \vec{k}+\gamma^{0} m\right)
$$

e colocando ambos os lados ao quadrado, obtemos

$$
(3 E+g \mathcal{A})^{2}=9\left[(\vec{\alpha} \cdot \vec{k})^{2}+(\vec{\alpha} \cdot \vec{k})\left(\gamma^{0} m\right)+\left(\gamma^{0} m\right)(\vec{\alpha} \cdot \vec{k})+\left(\gamma^{0} m\right)^{2}\right]
$$

Calculando separadamente o primeiro termo do lado direito, temos que

$$
\begin{aligned}
(\vec{\alpha} \cdot \vec{k})^{2} & =\left(\alpha^{1} k^{1}+\alpha^{2} k^{2}+\alpha^{3} k^{3}\right)^{2} \\
& =\left(\alpha^{1} k^{1}\right)^{2}+\left(\alpha^{2} k^{2}\right)^{2}+\left(\alpha^{3} k^{3}\right)^{2}+k^{1} k^{2}\left\{\alpha^{1}, \alpha^{2}\right\}+k^{1} k^{3}\left\{\alpha^{1}, \alpha^{3}\right\}+k^{2} k^{3}\left\{\alpha^{2}, \alpha^{3}\right\} \\
& =\left(k^{1}\right)^{2}+\left(k^{2}\right)^{2}+\left(k^{2}\right)^{3} \\
& =\vec{k}^{2}
\end{aligned}
$$

onde usamos que $\left\{\alpha^{i}, \alpha^{j}\right\}=0$, para $i \neq j$ e $\left(\alpha^{i}\right)^{2}=1$. E calculando separadamente o segundo e terceiro termos:

$$
\begin{aligned}
(\vec{\alpha} \cdot \vec{k})\left(\gamma^{0} m\right)+\left(\gamma^{0} m\right)(\vec{\alpha} \cdot \vec{k}) & =m\left[k^{1}\left\{\alpha^{1}, \gamma^{0}\right\}+k^{2}\left\{\alpha^{2}, \gamma^{0}\right\}+k^{3}\left\{\alpha_{3}, \gamma^{0}\right\}\right] \\
& =0
\end{aligned}
$$

já que temos a propriedade $\left\{\gamma^{0}, \alpha^{i}\right\}=0$. Com isso,

$$
(3 E+g \mathcal{A})^{2}=9\left(\vec{k}^{2}+m^{2}\right)
$$

onde usamos que $\gamma^{0} \gamma^{0}=1$. Aplicando a raíz dos dois lados e isolando $E$, obtemos

$$
E=-\frac{1}{3} g \mathcal{A}+\sqrt{\vec{k}^{2}+m^{2}}
$$


Substituindo essa equação na equação (D.2.5), obtemos a relação

$$
\sqrt{\vec{k}^{2}+m^{2}}=\vec{\alpha} \cdot \vec{k}+\gamma^{0} m
$$

que será necessária para a dedução da equação (3.2.37) (podemos notar que o lado direito da equação é igual ao operador presente na equação (3.2.37), porém trocando $\vec{k} \rightarrow-i \nabla$ ).

Agora partimos da equação desejada

$$
\begin{aligned}
\psi^{\dagger}\left(-i \vec{\alpha} \cdot \nabla+\gamma^{0} m\right) \psi & =\frac{1}{V} \sum_{\vec{k}, \vec{k}^{\prime}, \lambda, \lambda^{\prime}} A^{\prime \dagger} u^{\prime \dagger} e^{i k^{\prime} \cdot x} c_{i}^{\dagger}\left(-i \delta_{i j} \vec{\alpha} \cdot \nabla+\gamma^{0} m \delta_{i j}\right) A u e^{-i k \cdot x} c_{j} \\
& =\frac{1}{V} \sum_{\vec{k}, \vec{k}^{\prime}, \lambda, \lambda^{\prime}}\left(-3 i \vec{\alpha} \cdot(i \vec{k})+3 \gamma^{0} m\right) A^{\prime \dagger} u^{\prime \dagger} u A e^{-i\left(k-k^{\prime}\right) \cdot x} \\
& =\frac{3}{V} \sum_{\vec{k}, \lambda}\left(+\vec{\alpha} \cdot \vec{k}+\gamma^{0} m\right) A^{\prime \dagger} A .
\end{aligned}
$$

Aplicando em um sistema composto por $\mathrm{N}$ quarks, teremos

$$
\begin{aligned}
\left\langle N\left|\psi^{\dagger}\left(-i \vec{\alpha} \cdot \nabla+\gamma^{0} m\right) \psi\right| N\right\rangle & =\frac{3}{V} \sum_{\vec{k}, \lambda}\left(+\vec{\alpha} \cdot \vec{k}+\gamma^{0} m\right)\left\langle N\left|A^{\prime \dagger} A\right| N\right\rangle \\
& =\frac{3}{V} \sum_{\vec{k}, \lambda}\left(+\vec{\alpha} \cdot \vec{k}+\gamma^{0} m\right) .
\end{aligned}
$$

Nesse ponto, usamos o resultado da equação (D.2.6), obtemos que

$$
\left\langle N\left|\psi^{\dagger}\left(-i \vec{\alpha} \cdot \nabla+\gamma^{0} m\right) \psi\right| N\right\rangle=\frac{3}{V} \sum_{\vec{k}, \lambda} \sqrt{\vec{k}^{2}+m^{2}}
$$

Usando a equação (D.1.7), temos

$$
\left\langle N\left|\psi^{\dagger}\left(-i \vec{\alpha} \cdot \nabla+\gamma^{0} m\right) \psi\right| N\right\rangle=3 \frac{\gamma_{Q}}{(2 \pi)^{3}} \int d^{3} k \sqrt{\vec{k}^{2}+m^{2}} .
$$

Escrevendo $\vec{k}$ em coordenadas esféricas e deixando explícito o índice de sabor dos quarks, ficamos com a relação

$$
\left(\psi^{q}\right)^{\dagger}\left(-i \vec{\alpha} \cdot \nabla+\gamma^{0} m_{q}\right) \psi^{q} \rightarrow 3 \frac{\gamma_{Q}}{(2 \pi)^{3}} \int_{0}^{k_{F, q}} d k \vec{k}_{q}^{2} \sqrt{\vec{k}_{q}^{2}+m_{q}^{2}},
$$

que é a equação (3.2.37) que queríamos provar.

\section{D.3 Equação (3.2.45)}

Para provar a equação (3.2.45), precisamos antes obter duas relações necessárias. Para a primeira delas, partimos da equação (D.2.6) e aplicamos $u$ pela direita e $u^{\dagger} \gamma^{0}$ pela esquerda,

$$
u^{\dagger} \gamma^{0}(\vec{\alpha} \cdot \nabla) u=u^{\dagger} \gamma^{0} \sqrt{\vec{k}^{2}+m^{2}} u
$$


Em seguida, pegamos novamente a equação (D.2.6), porém aplicamos $u^{\dagger}$ pela esquerda e $\gamma^{0} u$, resultando em

$$
u^{\dagger}(\vec{\alpha} \cdot \nabla) \gamma^{0} u=u^{\dagger} \sqrt{\vec{k}^{2}+m^{2}} \gamma^{0} u
$$

Somando estes dois últimos resultados, obtemos

$$
u^{\dagger}\left[\left(\gamma^{0} \vec{\alpha}+\vec{\alpha} \gamma^{0} \gamma^{0}\right) \cdot \vec{k}+2 m\right] u=2 u^{\dagger} \gamma^{0} \sqrt{\vec{k}^{2}+m^{2}} u
$$

Usando a propriedade de que $\left\{\gamma^{0}, \alpha^{i}\right\}=0$, obtemos

$$
u^{\dagger} u m=\bar{u} u \sqrt{\vec{k}^{2}+m^{2}} .
$$

Isolando $\bar{u} u$, chegamos na relação

$$
\bar{u} u=\frac{m}{\sqrt{\vec{k}^{2}+m^{2}}} u^{\dagger} u,
$$

que é a primeira relação necessária para provar a equação (3.2.45).

Para obtermos a segunda relação necessária, partimos da equação (D.2.4), que podemos escrevê-la como

$$
\frac{1}{V} \sum_{\vec{k}, \lambda}(3 E+g \mathcal{A})=\frac{1}{V} \sum_{\vec{k}, \lambda}\left(3 \vec{\alpha} \cdot \vec{k}+3 \gamma^{0} m\right) .
$$

Aplicando $u$ pela direita e substituindo a equação (D.2) nesta última, obtemos

$$
\frac{1}{V} \sum_{\vec{k}, \lambda}\left(3 \sqrt{\vec{k}^{2}+m^{2}}\right) u=\frac{1}{V} \sum_{\vec{k}, \lambda}\left(3 \vec{\alpha} \cdot \vec{k}+3 \gamma^{0} m\right) u .
$$

Rearranjando os termos, ficamos com

$$
\frac{1}{V} \sum_{\vec{k}, \lambda}(\vec{\alpha} \cdot \vec{k}) u=\frac{1}{V} \sum_{\vec{k}, \lambda}\left(\sqrt{\vec{k}^{2}+m^{2}}+\gamma^{0} m\right) u .
$$

Aplicando $u^{\dagger}$ pela esquerda,

$$
\frac{1}{V} \sum_{\vec{k}, \lambda} u^{\dagger}(\vec{\alpha} \cdot \vec{k}) u=\frac{1}{V} \sum_{\vec{k}, \lambda}\left(\sqrt{\vec{k}^{2}+m^{2}} u^{\dagger} u+m \bar{u} u\right) .
$$

Neste ponto, usamos a relação (D.3.3) obtida anteriorimente, de forma que esta última equação possa ser escrita como

$$
\begin{aligned}
\frac{1}{V} \sum_{\vec{k}, \lambda} u^{\dagger}(\vec{\alpha} \cdot \vec{k}) u & =\frac{1}{V} \sum_{\vec{k}, \lambda}\left(\sqrt{\vec{k}^{2}+m^{2}} u^{\dagger} u+m \frac{m}{\sqrt{\vec{k}^{2}+m^{2}}} u^{\dagger} u\right) \\
& =\frac{1}{V} \sum_{\vec{k}, \lambda} u^{\dagger} \frac{\vec{k}^{2}}{\sqrt{\vec{k}^{2}+m^{2}}} u,
\end{aligned}
$$


o que implica em

$$
\vec{\alpha} \cdot \vec{k} \rightarrow \frac{\vec{k}^{2}}{\sqrt{\vec{k}^{2}+m^{2}}}
$$

que é a segunda relação necessária para a dedução da equação (3.2.45).

Nesse ponto, partimos para o termo desejado

$$
\begin{aligned}
\psi^{\dagger}(-i \vec{\alpha} \cdot \nabla) \psi & =\psi_{i}^{\dagger}\left(-i \delta_{i j} \vec{\alpha} \cdot \nabla\right) \psi_{j} \\
& =\frac{1}{V} \sum_{\vec{k}, \vec{k}^{\prime}, \lambda, \lambda^{\prime}} u^{\prime \dagger} A^{\prime \dagger} c_{i}^{\dagger} \delta_{i j} \vec{\alpha} \cdot \vec{k} c_{j} A u e^{-i\left(k-k^{\prime}\right) \cdot x} \\
& =\frac{3}{V} \sum_{\vec{k}, \vec{k}^{\prime}, \lambda, \lambda^{\prime}} u^{\prime \dagger} A^{\prime \dagger} \vec{\alpha} \cdot \vec{k} A u e^{-i\left(k-k^{\prime}\right) \cdot x} .
\end{aligned}
$$

Aplicando em um sistema com N quarks e usando a relação (D.3.4),

$$
\begin{aligned}
\left\langle N\left|\psi^{\dagger}(-i \vec{\alpha} \cdot \nabla) \psi\right| N\right\rangle & =\frac{3}{V} \sum_{\vec{k}, \lambda} \frac{\vec{k}^{2}}{\sqrt{\vec{k}^{2}+m^{2}}}\left\langle N\left|A^{\dagger \dagger} A\right| N\right\rangle \\
& =\frac{3}{V} \sum_{\vec{k}, \lambda} \frac{\vec{k}^{2}}{\sqrt{\vec{k}^{2}+m^{2}}} .
\end{aligned}
$$

Usando a relação (D.1.7), escrevendo $k$ em coordenadas esféricas e deixando explícito o índice de sabor dos quarks, obtemos a equação desejada

$$
\left(\psi^{q}\right)^{\dagger}(-i \vec{\alpha} \cdot \nabla) \psi^{q} \rightarrow 3 \frac{\gamma_{Q}}{2 \pi^{2}} \int_{0}^{k_{F, q}} d k \frac{\vec{k}_{q}^{4}}{\sqrt{\vec{k}_{q}^{2}+m_{q}^{2}}} .
$$





\section{Bibliografia}

[1] Victor Paulo Goncalves e Lucas da Silva Lazzari. "Uma introdução às estrelas estranhas". Em: Revista Brasileira de Ensino de Física (2020). ISSN: 1806-9126. DOI: 10 . 1590/1806-9126-rbef-2020-0032. arXiv: 2006.02976 [hep-ph].

[2] J. Adams et al. "Experimental and theoretical challenges in the search for the quark-gluon plasma: The STAR Collaboration's critical assessment of the evidence from RHIC collisions". Em: Nuclear Physics A 757.1 (2005). First Three Years of Operation of RHIC, pp. 102-183. ISSN: 0375-9474. DOI: $10.1016 / \mathrm{j}$. nuclphysa.2005.03.085. arXiv: nucl-ex/0501009.

[3] K. Hebeler et al. "Equation of State and Neutron Star Properties Constrained by Nuclear Physics and Observation”. Em: The Astrophysical Journal 773.1 (jul. de 2013), p. 11. ISSN: 1538-4357. DOI: 10 . 1088/0004-637x/773/1/11. arXiv: 1303.4662 [astro-ph.SR] .

[4] Toru Kojo. QCD equations of state and speed of sound in neutron stars. 2021. arXiv: 2011.10940 [nucl-th].

[5] Aleksi Kurkela, Paul Romatschke e Aleksi Vuorinen. "Cold quark matter". Em: Phys. Rev. D 81 (10 mai. de 2010), p. 105021. DoI: 10.1103 / PhysRevD 81. 105021. arXiv: 0912.1856 [hep-ph].

[6] Barry A. Freedman e Larry D. McLerran. "Fermions and gauge vector mesons at finite temperature and density. III. The ground-state energy of a relativistic quark gas”. Em: Phys. Rev. D 16 (4 ago. de 1977), pp. 1169-1185. DoI: $10.1103 /$ PhysRevD.16.1169.

[7] Eemeli Annala. "From QCD to Neutron Stars and Back : Probing the Fundamental Properties of Dense Matter". Tese de dout. Helsinki U., 2020. 
[8] Norman K. Glendenning. Compact Stars: Nuclear Physics, Particle Physics, and General Relativity. Springer, 2000.

[9] J. R. Oppenheimer e G. M. Volkoff. “On Massive Neutron Cores”. Em: Phys. Rev. 55 (4 fev. de 1939), pp. 374-381. DOI: 10.1103 / PhysRev. 55.374.

[10] Richard C. Tolman. "Static Solutions of Einstein's Field Equations for Spheres of Fluid". Em: Phys. Rev. 55 (4 fev. de 1939), pp. 364-373. DOI: 10.1103 / PhysRev. 55.364.

[11] M. B. Albino, R. Fariello e F. S. Navarra. "Tidal deformability of quark stars with repulsive interactions". Em: Physical Review D 104.8 (out. de 2021). ISSN: 2470-0029. DOI: 10.1103 / physrevd. 104.083011 . arXiv: 2106.12956 [nucl-th].

[12] B. Franzon et al. "Self-bound interacting QCD matter in compact stars". Em: Physical Review D 86.6 (set. de 2012). ISSN: 1550-2368. DOI: 10.1103 / physrevd. 86.065031. arXiv: 1203.6090 [astro-ph.SR].

[13] Salil Joshi, Sovan Sau e Soma Sanyal. "Quark cores in extensions of the MIT bag model". Em: Journal of High Energy Astrophysics 30 (jun. de 2021), pp. 16-23. ISSN: 2214-4048. DOI: $10.1016 / j$. jheap. 2021.03 .001 arXiv: 2002. 07647 [nucl-th].

[14] Luiz L Lopes, Carline Biesdorf e Débora P Menezes. "Modified MIT bag Models-part I: Thermodynamic consistency, stability windows and symmetry group". Em: Physica Scripta 96.6 (mar. de 2021), p. 065303. ISSN: 1402-4896. DOI: 10 . 1088/1402-4896/abef34. arXiv: 2005.13136 [hep-ph] .

[15] Luiz L Lopes et al. "Modified MIT Bag Models-part II: QCD phase diagram and hot quark stars". Em: Physica Scripta 96.6 (mar. de 2021), p. 065302. ISSN: 1402-4896. DOI: 10 . 1088/1402-4896/abef35. arXiv: 2009.13552 [hep-ph].

[16] K. Hebeler et al. "Constraints on Neutron Star Radii Based on Chiral Effective Field Theory Interactions". Em: Physical Review Letters 105.16 (out. de 2010). ISSN: 1079-7114. DOI: 10.1103/physrevlett.105.161102. arXiv: 1007 . 1746 [nucl-th].

[17] Sophia Han e Andrew W. Steiner. "Tidal deformability with sharp phase transitions in binary neutron stars". Em: Physical Review D 99.8 (abr. de 2019). ISSN: 2470-0029. DOI: 10.1103 / physrevd.99.083014. arXiv: 1810.10967 [nucl-th]. 
Natureza da transição de fase quark-hádron e consequências para a estrutura estelar

[18] Takuya Minamikawa, Toru Kojo e Masayasu Harada. "Quark-hadron crossover equations of state for neutron stars: Constraining the chiral invariant mass in a parity doublet model". Em: Physical Review C 103.4 (abr. de 2021). ISSN: 24699993. DOI: 10 .1103/physrevc.103 . 045205. arXiv: 2011.13684 [nucl-th].

[19] L. Tonetto e G. Lugones. "Discontinuity gravity modes in hybrid stars: Assessing the role of rapid and slow phase conversions". Em: Physical Review D 101.12 (jun. de 2020). ISSN: 2470-0029. DOI: 10 . 1103 / physrevd.101.123029. arXiv: 2003.01259 [astro-ph.HE] .

[20] Alessandro Parisi et al. Hybrid stars in the light of the merging event GW170817. 2020. arXiv: 2009.14274 [astro-ph.HE] .

[21] S. Schramm, V. Dexheimer e R. Negreiros. "Modelling hybrid stars in quarkhadron approaches". Em: The European Physical Journal A 52.1 (jan. de 2016). ISSN: 1434-601X. DOI: 10 .1140/epja/i2016-16014-5. arXiv: 1508 . 04699 [nucl-ph].

[22] F. Weber. "Strange quark matter and compact stars". Em: Progress in Particle and Nuclear Physics 54.1 (2005), pp. 193-288. ISSN: 0146-6410. DOI: 10.1016 / j . ppnp.2004.07.001. arXiv: astro-ph/0407155.

[23] P. B. Demorest et al. "A two-solar-mass neutron star measured using Shapiro delay". Em: Nature 467.7319 (out. de 2010), pp. 1081-1083. ISSN: 1476-4687. DOI: 10.1038 /nature09466. arXiv: 1010.5788 [astro-ph.HE] .

[24] Zaven Arzoumanian et al. "The NANOGrav 11-year Data Set: High-precision Timing of 45 Millisecond Pulsars". Em: The Astrophysical Journal Supplement Series 235.2 (abr. de 2018), p. 37. ISSN: 1538-4365. DOI: $10.3847 / 1538-4365 /$ aab5b0. arXiv: 1801.01837 [astro-ph.HE] .

[25] J. Antoniadis et al. "A Massive Pulsar in a Compact Relativistic Binary". Em: Science 340.6131 (abr. de 2013), pp. 1233232-1233232. ISSN: 1095-9203. DOI: $10.1126 /$ science.1233232. arXiv: 1304.6875 [astro-ph.HE] .

[26] H. T. Cromartie et al. "Relativistic Shapiro delay measurements of an extremely massive millisecond pulsar". Em: Nature Astronomy 4.1 (set. de 2019), pp. 72-76. ISSN: 2397-3366. DOI: 10 . 1038/s41550-019-0880-2. arXiv: 1904 . 06759 [astro-ph.HE].

[27] Thomas E. Riley et al. A NICER View of the Massive Pulsar PSR J0740+6620 Informed by Radio Timing and XMM-Newton Spectroscopy. 2021. arXiv: 2105. 06980 [astro-ph.HE]. 
[28] R. Abbott et al. "GW190814: Gravitational Waves from the Coalescence of a 23 Solar Mass Black Hole with a 2.6 Solar Mass Compact Object". Em: The Astrophysical Journal 896.2 (jun. de 2020), p. L44. ISSN: 2041-8213. DOI: 10 . 3847 / 2041-8213/ab960f. arXiv: 2006.12611 [astro-ph.HE] .

[29] I. Bombaci et al. "Was GW190814 a Black Hole-Strange Quark Star System?" Em: Physical Review Letters 126.16 (abr. de 2021). ISSN: 1079-7114. DOI: 10 . 1103 /physrevlett.126.162702. arXiv: 2010.01509 [nucl-th].

[30] Hung Tan, Jacquelyn Noronha-Hostler e Nico Yunes. "Neutron Star Equation of State in Light of GW190814". Em: Physical Review Letters 125.26 (dez. de 2020). ISSN: 1079-7114. DOI: 10.1103 /physrevlett.125.261104.arXiv: 2006. 16296 [astro-ph.HE].

[31] M. C. Miller et al. "PSR J0030+0451 Mass and Radius from NICER Data and Implications for the Properties of Neutron Star Matter". Em: The Astrophysical Journal 887.1 (dez. de 2019), p. L24. DOI: 10 . 3847 / $2041-8213$ / ab 50 c5. arXiv: 1912.05705 [astro-ph.HE].

[32] T. E. Riley et al. "A NICER View of PSR J0030+0451: Millisecond Pulsar Parameter Estimation". Em: The Astrophysical Journal 887.1 (dez. de 2019), p. L21. ISSN: 2041-8213. DOI: $10.3847 / 2041-8213 /$ ab 481 c. arXiv: 1912.05702 [astro-ph.HE].

[33] Slavko Bogdanov et al. "Constraining the Neutron Star Mass-Radius Relation and Dense Matter Equation of State with NICER. I. The Millisecond Pulsar X-Ray Data Set". Em: The Astrophysical Journal 887.1 (dez. de 2019), p. L25. DOI: 10 . $3847 / 2041-8213 /$ ab53eb. arXiv: 1912.05706 [astro-ph.HE] .

[34] B. P. Abbott et al. "GW170817: Observation of Gravitational Waves from a Binary Neutron Star Inspiral”. Em: Physical Review Letters 119.16 (out. de 2017). ISSN: 1079-7114. DOI: 10.1103/physrevlett.119.161101. arXiv: 1710 . $05832[\mathrm{gr}-\mathrm{qC}]$.

[35] B. P. Abbott et al. "GW170817: Measurements of Neutron Star Radii and Equation of State”. Em: Physical Review Letters 121.16 (out. de 2018). ISSN: 10797114. DOI: $10.1103 /$ physrevlett.121.161101. arXiv: 1805.11581 $[g r-q C]$.

[36] D.A. Fogaça e F.S. Navarra. "Gluon condensates in a cold quark-gluon plasma". Em: Physics Letters B 700.3-4 (jun. de 2011), pp. 236-242. ISSN: 0370-2693. DOI: $10.1016 / j \cdot$ physletb.2011.05.011. arXiv: 1012.5266 [hep-ph] . 
[37] Yogiro Hama et al. "3D Relativistic Hydrodynamic Computations Using LatticeQCD-Inspired Equations of State". Em: Nuclear Physics A 774 (ago. de 2006), pp. 169-178. ISSN: 0375-9474. DOI: $10.1016 / \mathrm{j}$. nuclphysa. 2006.06 . 024. arXiv: hep-ph/0510096.

[38] Y. Hama. "Effects of LatticeQCD EoS and Continuous Emission on Some Observables”. Em: AIP Conference Proceedings (2006). ISSN: 0094-243X. DOI: 10 . $1063 / 1.2197459$. arXiv: hep-ph/0510101.

[39] Wen-Hua Cai e Qing-Wu Wang. Exploring the parameter space of quasi-particle model with the strange quark stars. 2020. arXiv: 2010.07448 [hep-ph].

[40] Tanja Hinderer. "Tidal Love Numbers of Neutron Stars". Em: The Astrophysical Journal 677.2 (abr. de 2008), pp. 1216-1220. ISSN: 1538-4357. DOI: 10.1086 / 533487. arXiv: 0711.2420 [astro-ph].

[41] Andrea Sabatucci. "Tidal deformation of neutron stars". Roma, Itália: Universidade de Roma "La Sapienza", 2018.

[42] A. R. Bodmer. “Collapsed Nuclei”. Em: Phys. Rev. D 4 (6 set. de 1971), pp. 16011606. DOI: $10.1103 /$ PhysRevD .4.1601.

[43] Edward Witten. "Cosmic separation of phases". Em: Phys. Rev. D 30 (2 jul. de 1984), pp. 272-285. DOI: 10.1103 /PhysRevD.30.272.

[44] Hidezumi Terazawa. "Super-Hypernuclei in the Quark-Shell Model". Em: Journal of the Physical Society of Japan 58.10 (1989), pp. 3555-3563. DOI: $10.1143 /$ JPS J. 58.3555.

[45] Simon Weissenborn et al. "QUARK MATTER IN MASSIVE COMPACT STARS”. Em: The Astrophysical Journal 740.1 (set. de 2011), p. L14. DOI: 10 . 1088 / 2041-8205/740/1/114.

[46] Edward Farhi e R. L. Jaffe. "Strange matter". Em: Phys. Rev. D 30 (11 dez. de 1984), pp. 2379-2390. DOI: 10.1103/PhysRevD . 30.2379.

[47] H. David Politzer. "Reliable Perturbative Results for Strong Interactions?" Em: Phys. Rev. Lett. 30 (26 jun. de 1973), pp. 1346-1349. DoI: 10.1103 / Phy sRevLett. 30.1346.

[48] David J. Gross e Frank Wilczek. "Ultraviolet Behavior of Non-Abelian Gauge Theories”. Em: Phys. Rev. Lett. 30 (26 jun. de 1973), pp. 1343-1346. DOI: $10.1103 /$ PhysRevLett.30.1343.

[49] Mark Thomson. Modern Particle Physics. Cambridge University Press, 2013. 
[50] Danuce M. Dudek. "Efeitos da equação de estado em hidrodinâmica relativística através de alguns observáveis". Tese de dout. São Paulo, Brasil: Universidade de São Paulo, 2014.

[51] Bruno Cezar de Souza Franzon. "Gluons em Estrelas de Nêutrons”. São Paulo, Brasil: Universidade de São Paulo, 2012.

[52] Samuel Mendes Sanches Júnior. "Equações de Estado do Plasma de Quarks e Glúons e suas Aplicações”. São Paulo, Brasil: Universidade de São Paulo, 2018.

[53] L. S. Celenza e C. M. Shakin. "Description of the gluon condensate". Em: Phys. Rev. D 34 (5 set. de 1986), pp. 1591-1600. DoI: 10.1103 / PhysRevD . 34 . 1591.

[54] Xiangdong Li e C. M. Shakin. "Description of gluon propagation in the presence of an $A^{2}$ condensate". Em: Phys. Rev. D 71 (7 abr. de 2005), p. 074007. DOI: 10.1103/PhysRevD.71.074007. arXiv: hep-ph/0410404.

[55] B.D. Serot e J.D. Walecka. Advances in Nuclear Physics. Vol. 16. 1986.

[56] Hirokazu Tezuka. "Mean Field Approximation to QCD. 1. Selfconsistent Equations of Motion". Em: (set. de 1987).

[57] Mark Alford et al. "Hybrid Stars that Masquerade as Neutron Stars". Em: The Astrophysical Journal 629.2 (ago. de 2005), pp. 969-978. ISSN: 1538-4357. DOI: $10.1086 / 430902$. arXiv: nucl-th/0411016.

[58] Andreas Schmitt. "Dense Matter in Compact Stars". Em: Lecture Notes in Physics (2010). ISSN: 1616-6361. DOI: 10 . 1007/978-3-642-12866-0. arXiv: 1001.3294 [astro-ph.SR].

[59] Irina Sagert et al. "Compact stars for undergraduates". Em: European Journal of Physics 27.3 (abr. de 2006), pp. 577-610. ISSN: 1361-6404. DOI: 10 . $1088 /$ 0143-0807/27/3/012. arXiv: astro-ph/0506417.

[60] Gordon Baym, Christopher Pethick e Peter Sutherland. "The Ground state of matter at high densities: Equation of state and stellar models”. Em: Astrophys. J. 170 (1971), pp. 299-317. DOI: $10.1086 / 151216$.

[61] F. Douchin e P. Haensel. "A unified equation of state of dense matter and neutron star structure". Em: Astronomy \& Astrophysics 380.1 (dez. de 2001), pp. 151-167. ISSN: 1432-0746. DOI: 10 .1051/0004-6361:20011402. arXiv: astroph/ 0111092.

[62] Jocelyn S. Read et al. "Constraints on a phenomenologically parametrized neutronstar equation of state". Em: Physical Review D 79.12 (jun. de 2009). DOI: 10 . 1103 /physrevd.79.124032. arXiv: 0812.2163 [astro-ph]. 
[63] Michael F. O'Boyle et al. "Parametrized equation of state for neutron star matter with continuous sound speed". Em: Phys. Rev. D 102.8 (2020), p. 083027. DOI: $10.1103 /$ PhysRevD.102.083027. arXiv: 2008.03342 [astro-ph.HE] .

[64] Wasif Husain e Anthony W. Thomas. "Hybrid stars with hyperons and strange quark matter". Em: PROCEEDINGS OF THE 14TH ASIA-PACIFIC PHYSICS CONFERENCE (2021). DOI: 10.1063/5.0036994. arXiv: 2010.06750 [hep-ph].

[65] Rone P. G. Andrade. "Sobre a evolução hidrodinâmica da matéria nuclear criada em colisões de íons pesados relativísticos - um estudo com condições iniciais flutuantes”. Tese de dout. São Paulo, Brasil: Universidade de São Paulo, 2011.

[66] Bryen Irving. "Relativistic Tidal Deformations in Hybrid Stars". Diss. de mestr. California State University, Long Beach, jan. de 2019.

[67] Sean Carroll. Spacetime and Geometry: An Introduction to General Relativity. Benjamin Cummings, 2003. ISBN: 0805387323.

[68] James B. Hartle. Gravity: An Introduction to Einstein's General Relativity. AddisonWesley Professional, 2003. ISBN: 0805386629.

[69] Tullio Regge e John A. Wheeler. "Stability of a Schwarzschild Singularity". Em: Phys. Rev. 108 (4 nov. de 1957), pp. 1063-1069. DOI: 10.1103 / PhysRev . 108.1063.

[70] Kip S. Thorne e Alfonso Campolattaro. "Non-Radial Pulsation of General-Relativistic Stellar Models. I. Analytic Analysis for $L \geq 2$ ". Em: The Astrophysical Journal 149 (set. de 1967), p. 591. DOI: $10.1086 / 149288$.

[71] Tanja Hinderer et al. "Tidal deformability of neutron stars with realistic equations of state and their gravitational wave signatures in binary inspiral". Em: Physical Review D 81.12 (jun. de 2010). ISSN: 1550-2368. DOI: 10.1103 / physrevd. 81.123016. arXiv: 0911.3535 [astro-ph.HE].

[72] Anthony Kalaitzis. "Constraining the Equation of State of Nuclear Matter with Neutron Stars". Adelaide, Austrália: The University of Adelaide, 2020. 